\title{
Sheaves on Toric Varieties for Physics
}

\author{
Allen Knutson ${ }^{a}$, Eric Sharpe ${ }^{b}$ \\ ${ }^{a}$ Mathematics Department \\ Brandeis University \\ Waltham, MA 02254 \\ allenk@alumni.caltech.edu \\ ${ }^{b}$ Physics Department \\ Princeton University \\ Princeton, NJ 08544 \\ ersharpe@puhep1.princeton.edu
}

\begin{abstract}
In this paper we give an inherently toric description of a special class of sheaves (known as equivariant sheaves) over toric varieties, due in part to A. A. Klyachko. We apply this technology to heterotic compactifications, in particular to the $(0,2)$ models of Distler, Kachru, and also discuss how knowledge of equivariant sheaves can be used to reconstruct information about an entire moduli space of sheaves. Many results relevant to heterotic compactifications previously known only to mathematicians are collected here - for example, results concerning whether the restriction of a stable sheaf to a Calabi-Yau hypersurface remains stable are stated. We also describe substructure in the Kähler cone, in which moduli spaces of sheaves are independent of Kähler class only within any one subcone. We study F theory compactifications in light of this fact, and also discuss how it can be seen in the context of equivariant sheaves on toric varieties. Finally we briefly speculate on the application of these results to $(0,2)$ mirror symmetry.
\end{abstract}

e-print archive: http://xxx.lanl.gov/abs/hep-th/9711036 


\section{Introduction}

Historically one of the biggest challenges facing anyone wishing to study heterotic compactifications has been the lack of a good description of vector bundles and, more generally, torsion-free sheaves.

This problem has been recently addressed for the special case of vector bundles over elliptic varieties in $[29,30,31,32,33,34,13,35]$. However, their methods can neither be applied to more general varieties nor to study more general sheaves.

Most Calabi-Yaus studied to date have been complete intersections in toric varieties ${ }^{1}$. A description of sheaves relevant to all such Calabi-Yaus would be of tremendous importance.

In $[20,17]$ attempts were made to study sheaves on such Calabi-Yaus by describing the sheaves with short exact sequences and monads. These methods are somewhat clumsy and have not been thoroughly developed in the literature. For example, to date these methods have not given a global description of moduli spaces of sheaves, one of several prerequisites needed to gain a solid understanding of heterotic compactifications.

In this paper we present a radically different method of studying sheaves over Calabi-Yaus realized as complete intersections in toric varieties. In particular, we present an inherently toric method to describe certain sheaves on an ambient toric variety, due largely to Alexander A. Klyachko. Sheaves over a complete intersection Calabi-Yau can be obtained by restricting sheaves on the ambient space to the complete intersection. This does not allow us to realize all possible sheaves over a Calabi-Yau, but it does still give a tremendous amount of insight into moduli spaces. In particular, it is closely analogous to the strategy of studying the Kähler cone of a complete intersection Calabi-Yau via the Kähler cone of the ambient toric variety, used to great effect in the mirror symmetry literature.

Not any sheaf over a Calabi-Yau can be used in a compactification one restriction is that the sheaf must be stable. We are able to determine explicitly whether a bundle on the ambient toric variety is stable, but unfortunately the restriction of a stable sheaf to a hypersurface is not, in general, stable $^{2}$. However, in special cases the restriction of a stable sheaf to a hypersurface is stable; sufficient conditions will be discussed in section 8 .

\footnotetext{
${ }^{1}$ For introductions to toric varieties see for example $[8,9,10,11,12]$.

${ }^{2}$ with respect to restriction of the Kähler form
} 
The condition that a sheaf be stable implicitly depends upon the choice of Kähler form. It sometimes happens that a sheaf is stable with respect to some, but not all, of the possible Kähler forms in the Kähler cone. In the more extreme versions of this phenomenon the Kähler cone is subdivided into chambers, each associated to distinct moduli spaces of sheaves. This phenomenon will be discussed in section 6 .

Although we have a lot to say about classical results, we largely ignore quantum effects. For example, we do not speak to worldsheet instanton corrections. These are a very important aspect of heterotic compactifications, but we do not at present have sufficient technology to deeply understand their effects. Perhaps the only exception to this are some speculations on the application of the results in this paper to $(0,2)$ mirror symmetry, in section 10. Our philosophy in writing this paper was to first try to understand classical behavior, and leave quantum corrections to future work.

In this paper a great deal of mathematics is used which is unfamiliar to most physicists, even those who often think about compactifications. To help alleviate the resulting culture shock, we have tried to loosely order this paper so that topics requiring less mathematical sophistication appear near the beginning, and those requiring more appear later. Throughout the paper, we have made an effort to explain nearly all of the mathematics used, even to the extent of including lengthy elaborations (where useful) of details that some would consider basic. Extremely technical details, not elaborated upon in detail, are usually banished to footnotes. We have also provided several appendices on the basics of relevant mathematical topics, which ideally should help the reader understand not only this paper but also parts of the mathematics literature cited.

In section 2 we begin with a short review of constraints on consistent heterotic compactifications. In section 3 we make general remarks on sheaves on toric varieties, which should help put the rest of the paper in context. In section 4 we discuss equivariant sheaves on toric varieties. We discuss not only how to construct equivariant bundles (of any structure group) and more general sheaves, but also the calculation of invariants such as Chern classes, sheaf cohomology groups, and global Ext groups. We also discuss equivariant sheaves on singular varieties. In section 5 we explicitly construct moduli spaces of equivariant sheaves, and discuss the underlying theory in some detail. In section 6 we discuss substructure in the Kähler cone, both in generality and in the special cases of elliptic K3's with section (relevant to $F$ theory) and to equivariant sheaves on toric surfaces. In section 7 we discuss more general moduli spaces, of not-necessarily-equivariant sheaves. In particular, we discuss how knowledge of equivariant sheaves can be used 
to reconstruct information about the rest of the moduli space. We also explicitly construct complete moduli spaces of bundles on $\mathbf{P}^{2}$ (one of the few cases that can be understood explicitly), and as an aside derive the ADHM construction. Up to this point we have only discussed sheaves on ambient toric varieties; in section 8 we discuss conditions under which the restriction of a stable sheaf to a hypersurface is stable. In section 9 we apply the technology above to the $(0,2)$ models of Distler and Kachru. In section 10 we apply the technology above to make nontrivial statements about $(0,2)$ mirror symmetry. Finally, we conclude in section 11 with speculations. Four appendices review basic facts about algebraic groups, sheaf theory, GIT quotients, and filtrations commonly used in sheaf theory.

Much of this paper is devoted to reviewing highly relevant results known to mathematicians but not physicists. For example, an inherently toric description of equivariant sheaves was originally worked out by A. A. Klyachko $[1,2,3,4]$, but in order to make his results useful for physicists, we have had to significantly extend them. Since his work is also completely unknown to physicists (despite its great relevance), we have taken this opportunity to review it, and in places correct it. Similarly, results on stability of the restriction of a stable sheaf to a hypersurface exist in the mathematics literature but are unknown to the physicists - so we have presented known results here. In fact, for convenience we have collected here many results highly relevant to heterotic compactifications that were previously known only to mathematicians.

We should also mention a few sections can be read independently of the rest of the paper. Section 8, on stability of restrictions of stable sheaves to hypersurfaces, can be read apart from the rest. The first two-thirds of section 6 , on Kähler cone substructure sensed by heterotic theories, can be read independent of the rest of the paper.

A word of caution is in order concerning conventions used in this paper. We will assume the structure groups of all bundles are reductive algebraic groups - complexifications of compact Lie groups. In particular, instead of working with $U(n)$, we shall work with $G L(n, \mathbf{C})$, its complexification; similarly, instead of $S U(n)$, we work with $S L(n, \mathbf{C})$.

\section{A Rapid Review of Heterotic Compactifications}

For a consistent perturbative compactification of either the $E_{8} \times E_{8}$ or $\operatorname{Spin}(32) / \mathbf{Z}_{2}$ heterotic string, in addition to specifying a Calabi-Yau $Z$ one 
must also specify a set of holomorphic vector bundles (or, more generally, sheaves) $V_{i}$. These vector bundles must obey two constraints. For $G L(n, \mathbf{C})$ bundles one constraint ${ }^{3}$ can be written as

$$
\omega^{n-1} \cup c_{1}\left(V_{i}\right)=0
$$

where $n$ is the complex dimension of the Calabi-Yau, and $\omega$ is the Kähler form. This constraint has a somewhat subtle implication. In general, for any holomorphic bundle $\mathcal{E}$, if there exists a Hermitian connection associated to $\mathcal{E}$ such that, in every coordinate chart, the curvature $F$ satisfies $F \wedge \omega^{n-1}=c I$, where $I$ is the identity matrix and $c \in \mathbf{R}$ is a fixed chart-independent constant, then $\mathcal{E}$ is either properly Mumford-Takemoto stable [38] or MumfordTakemoto semistable and split $[26,27,28]$. Thus, the constraint in equation (1) implies that (but is not equivalent to the statement) $\mathcal{E}$ is either stable, or semistable and split. In fact, we can slightly simplify this statement. Properly semistable sheaves are grouped ${ }^{4}$ in $S$-equivalence classes, and each $S$-equivalence class contains a unique split representative [80, p. 23]. Thus, constraint (1) implies that $\mathcal{E}$ is Mumford-Takemoto semistable.

The other constraint is an anomaly-cancellation condition which, if a single $G L(n, \mathbf{C})$ bundle $V_{i}$ is embedded in each $E_{8}$, is often ${ }^{5}$ written as

$$
\sum_{i}\left(c_{2}\left(V_{i}\right)-\frac{1}{2} c_{1}\left(V_{i}\right)^{2}\right)=c_{2}(T Z)
$$

It was noted [24] that the anomaly-cancellation conditions can be modified slightly by the presence of five-branes in the heterotic compactification. Let $[W]$ denote the cohomology class of the five-branes, then the second constraint above is modified to

$$
\sum_{i}\left(c_{2}\left(V_{i}\right)-\frac{1}{2} c_{1}\left(V_{i}\right)^{2}\right)+[W]=c_{2}(T Z)
$$

Although the conditions above are necessary for a consistent heterotic compactification, they are not sufficient - quantum effects must also be taken

\footnotetext{
${ }^{3}$ For example, for compactifications to four dimensions, $N=1$ supersymmetry, on a Calabi-Yau $X$ one gets a $D$ term in the low-energy effective action proportional to $\langle X| \omega^{2} U$ $\left.c_{1}(V)\right\rangle$.

${ }^{4}$ More precisely, points on a moduli space of sheaves that are properly semistable do not necessarily correspond to unique semistable sheaves, but rather to $S$-equivalence classes of properly semistable sheaves. Points that are stable do correspond to unique stable sheaves - $S$-equivalence classes are a phenomenon arising only for properly semistable objects.

${ }^{5}$ On rare occasion it is possible for a perturbative compactification to evade this condition. See for example [14].
} 
into account. For example, it was once believed that generic heterotic compactifications were destabilized by worldsheet instantons [18]. For the $(0,2)$ models of [17], it has been shown that this is not the case [19], and in fact it has become fashionable to ignore this difficulty. In addition, even for the $(0,2)$ models of [17], there is a more subtle and poorly-understood anomaly [22] which afflicts many potential heterotic compactifications. In this paper we will speak to neither potential problem; our philosophy is to first understand purely classical behavior, and only then attempt to grasp quantum corrections.

Historically heterotic compactifications used only bundles, not more general sheaves. However, in [23] it was shown that it was possible to have consistent perturbative heterotic compactifications involving sheaves (specifically, torsion-free sheaves) which are not bundles. There is, however, a caveat. On a smooth variety, all torsion-free sheaves look like bundles up to codimension at least two, where the description as a bundle breaks down. Because of these bad points, it sometimes happens that the conformal field theory breaks down - in such cases the metric degenerates and describes an infinite tube, sometimes loosely associated with five-branes [104, 105]. In order to determine whether a particular torsion-free sheaf suffers from this difficulty, one writes down a linear sigma model describing the sheaf and studies its properties as in [23]. Unfortunately at present there is no invariant method to determine whether a given torsion-free sheaf describes a singular conformal field theory - one must study an associated linear sigma model. Most of the sheaves we shall study in this paper are not obviously associated with linear sigma models, so we have no way to determine whether a given (non-locally-free) sheaf is associated with a singular conformal field theory. We feel that the advantages of our new approach to thinking about heterotic compactifications outweigh such difficulties.

\section{General Remarks on Bundles on Toric Varieties}

In this section we will make some general observations on moduli spaces of bundles (of fixed Chern classes) on a toric variety $X$. (In fact, our remarks will also hold for moduli spaces of reflexive, and torsion-free, sheaves.)

First, we should mention a few basic facts about toric varieties that will be used throughout this paper. A toric variety is a compactification ${ }^{6}$ of some "algebraic torus" $\left(\mathbf{C}^{\times}\right)^{n}$, where $\mathbf{C}^{\times}=\mathbf{C}-\{0\}$. For example, all projective

\footnotetext{
${ }^{6}$ Not all compactifications of algebraic tori are toric varieties - toric varieties have additional nice properties - but the distinctions will not be relevant for our discussion.
} 
spaces are toric varieties:

$$
\begin{aligned}
& \mathbf{P}^{1}=\mathbf{C}^{\times} \cup\{0\} \cup\{\infty\} \\
& \mathbf{P}^{2}=\left(\mathbf{C}^{\times}\right)^{2} \cup\{x=0\} \cup\{y=0\} \cup\{z=0\}
\end{aligned}
$$

where $x, y$, and $z$ are homogeneous coordinates defining $\mathbf{P}^{2}$, and so forth.

The codimension one subvarieties added to the algebraic torus to compactify it are known as the "toric divisors." For example, the toric divisors of $\mathbf{P}^{1}$ are $\{0\}$ and $\{\infty\}$. The toric divisors of $\mathbf{P}^{2}$ are $\{x=0\},\{y=0\}$, and $\{z=0\}$.

Note that the algebraic torus $\left(\mathbf{C}^{\times}\right)^{n}=T$ underlying any toric variety $X$ has a natural action on $X$. In the case of $\mathbf{P}^{1}$, this action amounts to rotations about an axis and dilations.

A moduli space of bundles (or sheaves) $\mathcal{M}$ also has a natural action of the algebraic torus $T$ defining the toric variety $X$ : if $\mathcal{E}$ is some sheaf and $t: X \rightarrow X$ the action of an element $t \in T$, then we take $\mathcal{E} \rightarrow t^{*} \mathcal{E}$. In general, $\mathcal{E} ¥ t^{*} \mathcal{E}$. The fixed-point locus of the $T$ action consists of sheaves $\mathcal{E}$ such that $t^{*} \mathcal{E} \simeq \mathcal{E}$ for all elements ${ }^{7} t \in T$. For example, all line bundles on a smooth toric variety have this property. Such sheaves are known as equivariant ${ }^{8}$ sheaves, or sometimes homogeneous sheaves. Such sheaves have an inherently toric description $[1,2,3,4,6,7]$, which we shall review and extend in the first part of this paper.

This inherently toric description of equivariant vector bundles and equivariant reflexive sheaves associates a filtration ${ }^{9}$ of a vector space to each toric

\footnotetext{
${ }^{7}$ Some toric varieties have automorphism groups larger than the algebraic torus [9] which might, in principle, give additional information. We will not pursue this possibility here.

${ }^{8}$ In fact, we are being slightly sloppy. In the mathematics literature, an "equivariant" sheaf would not only have the property that it is mapped into itself by all algebraic torus actions, but in addition would come with a fixed choice of "equivariant structure" (a precise choice of sheaf involution). Sheaves that are fixed under all algebraic torus actions but do not have a fixed equivariant structure should be called "equivariantizable." For reasons of readability, we shall maliciously fail to distinguish "equivariant" from "equivariantizable." For more information on the relationship between "equivariant" and "equivariantizable," see section 7 .

${ }^{9} \mathrm{~A}$ filtration of a vector space $V$ is a sequence of nested subspaces

$$
V=V_{0} \supseteq V_{1} \supseteq V_{2} \supseteq \cdots
$$

with a strictly increasing integer associated to each subspace. In the filtration description of equivariant vector bundles, the vector space we filter is precisely the fiber of the vector bundle. More general equivariant torsion-free sheaves are trivial vector bundles over the open $T$-orbit; the vector space we filter is that of the trivial vector bundle. This filtration description is valid for both bundles and reflexive sheaves on both smooth and singular
} 
divisor. Intuitively, equivariant sheaves on an algebraic torus $\left(\mathbf{C}^{\times}\right)^{n}$ are trivial, so all information is contained in their behavior near the toric divisors. Not all choices of filtrations define a bundle - to get a bundle, rather than merely a reflexive sheaf, there is a compatibility condition that must be satisfied. There is also a description of more general equivariant torsion-free sheaves, but this description is more cumbersome and so is presented later.

In order to use the description of equivariant sheaves outlined above, one must fix a precise action of the algebraic torus $T$ on the equivariant sheaf - it is not enough to know the fact that the algebraic torus maps the sheaf into itself, we must also specify precisely how the algebraic torus acts. Put another way, for any element $t$ of the algebraic torus $T$, there exists an action of $t$ on an equivariant sheaf $\mathcal{E}$ which makes the following diagram commute:

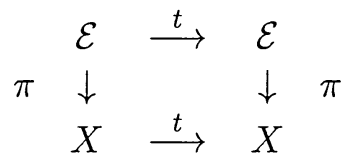

where $\pi: \mathcal{E} \rightarrow X$ is the projection. This choice of algebraic torus action, known as the choice of "equivariant structure," is not unique, and the filtration description outlined above depends upon the precise choice, but for all that the choice is actually quite harmless - it adds no continuous moduli, and is under good control.

The "inherently toric" description of sheaves outlined above only applies to equivariant sheaves, so what can we say about the rest of a moduli space of sheaves, given knowledge of only the equivariant ones? In principle, quite a lot. For any space with a torus action, given information about only the fixed points of a torus action, and the torus action on the normal bundle to the fixed points, it is possible to determine a great deal of information about the original space. In particular, even if we only know about equivariant sheaves we can still determine a great deal of information about a moduli space of not-necessarily-equivariant sheaves. This perspective will be reviewed in greater depth in section 7 .

Once we have discussed sheaves on ambient toric varieties in great detail, we turn to sheaves on Calabi-Yau hypersurfaces beginning in section 8 .

In most of the rest of this paper, we shall assume the reader is well acquainted with toric varieties and their associated machinery. For introductions to toric varieties see for example $[8,9,10,11,12]$.

toric varieties, but to aid the reader will will begin our discussion by restricting to the special case of bundles on smooth toric varieties. 


\section{Equivariant Sheaves}

In this section we will review results on equivariant sheaves - the sheaves located at the fixed points of the algebraic torus action on the moduli space.

In section 4.1 we begin by reviewing an inherently toric description of equivariant vector bundles, due originally to Alexander A. Klyachko $[1,2,3$, 4]. In section 4.2 we compare Klyachko's description to another, somewhat less useful, description due to Kaneyama [6, 7]. In section 4.3 we describe how to compute Chern classes and sheaf cohomology groups using Klyachko's description. In section 4.4 we describe how to modify Klyachko's description to describe bundles with arbitrary gauge group. Finally in section 4.5 we describe how to generalize Klyachko's description to give arbitrary torsionfree sheaves on arbitrary toric varieties. We derive Klyachko's description from first principles, discuss equivariant sheaves on singular varieties, give an efficient description of reflexive sheaves on arbitrary varieties, and on smooth toric varieties we describe global Ext calculations.

Many of the results in this section are due originally to A. A. Klyachko $[1,2,3,4]$, and are reviewed (and occasionally corrected) for the reader's convenience. In particular, much of sections 4.1, 4.3, 4.4, and 4.5.2 is due to A. A. Klyachko. In particular, A. A. Klyachko was concerned with smooth toric varieties, but in order to make these methods useful for physical applications we extended his work to singular varieties, to global Ext calculations, and (in the next section) to moduli space problems.

In sections 4.1 through 4.4 we specialize to smooth toric varieties and bundles on these varieties. More general toric varieties and more general sheaves are considered in section 4.5.

\subsection{Equivariant Vector Bundles}

In this section we will review work of A. A. Klyachko [1, 2, 3, 4] describing equivariant bundles on smooth toric varieties in an inherently toric fashion. The basic idea is that an equivariant bundle is completely determined by its behavior near the toric divisors. Thus, Klyachko specifies bundles in terms of a family of filtrations of a vector space, one filtration for each toric divisor. We will first describe the relevant technology, then afterwards try to give some intuitive understanding. Readers well-versed in algebraic geometry may find the discussion of equivariant torsion-free sheaves in section 4.5.1 somewhat more enlightening than the discussion in this section. 
In section 4.5 we speak about more general sheaves on toric varieties that are not necessarily smooth.

Klyachko describes equivariant vector bundles by associating to each toric divisor a filtration of the generic fiber. In order for these filtrations to yield a well-defined bundle, they must satisfy a certain compatibility condition, to be defined shortly. A set of (compatible) filtrations is sufficient to uniquely identify the equivariant bundle.

In order to use this filtration prescription one must make a specific choice of action of the algebraic torus on the bundle. Since the bundle is equivariant, the algebraic torus action maps the bundle into itself - we need to be specific about the choice of involution. More precisely, for any element $t$ of the algebraic torus $T$, there exists an action of $t$ on an equivariant bundle $\mathcal{E}$ which makes the following diagram commute:

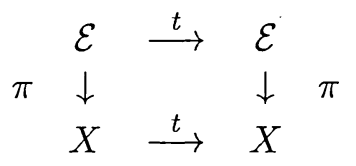

where $\pi: \mathcal{E} \rightarrow X$ is the projection. This choice of algebraic torus action, known as the choice of "equivariant structure," is not unique, and the filtration description outlined above depends upon the precise choice, but for all that the choice is actually quite harmless - it adds no continuous moduli, and is under good control. In the case of line bundles, the choice of equivariant structure is equivalent to a precise choice of $T$-invariant divisor. For example, on $\mathrm{P}^{2}$ the line bundles $\mathcal{O}\left(D_{x}\right), \mathcal{O}\left(D_{y}\right)$, and $\mathcal{O}\left(2 D_{z}-D_{y}\right)$, where $D_{x}=\{x=0\}$ and $x, y, z$ are homogeneous coordinates, are all equivalent as line bundles to $\mathcal{O}(1)$, but have distinct equivariant structures.

Now we shall describe how to obtain a set of filtrations given some bundle $\mathcal{E}$ over a toric variety $X$, following $[1,2,3,4]$. Fix a point $p_{0}$ in the open torus orbit, and define $E=\mathcal{E}\left(p_{0}\right)$. For each toric divisor $\alpha$, let $p_{\alpha}$ be a generic point in the toric divisor. We will obtain a filtration for the toric divisor $\alpha$ by observing how the fiber $E$ changes as we drag $p_{0} \rightarrow p_{\alpha}$. In particular, let $f(p)$ be any rational function on $X$ with a pole of order $i$ on the toric divisor $\alpha$, and $t$ a one-parameter algebraic torus action dragging $p_{0} \rightarrow p_{\alpha}$, then define

$$
E^{\alpha}(i)=\left\{e \in E \mid \lim _{t p_{0} \rightarrow p_{\alpha}} f\left(t p_{0}\right) \cdot(t e) \text { exists }\right\}
$$

These subspaces form a nonincreasing filtration:

$$
\cdots \supseteq E^{\alpha}(i) \supseteq E^{\alpha}(i+1) \supseteq \cdots
$$


with limits

$$
\begin{array}{ll}
E^{\alpha}(i)=0 & i \gg 0 \\
E^{\alpha}(i)=E & i \ll 0
\end{array}
$$

A random set of filtrations does not necessarily describe a vector bundle; these filtrations are required to satisfy a compatibility condition. This condition is simply that, for any cone $\sigma \in \Sigma$, the fan of $X$, the filtrations $E^{\alpha}(i)$, $\alpha \in|\sigma|$, consist of coordinate subspaces of some basis of the space $E$. Put another way, on any open set corresponding to a maximal cone, the vector bundle should split into a sum of line bundles. Put yet another way, vector bundles on affine space are trivial.

This compatibility condition can be phrased more abstractly as follows. For any toric divisor $\alpha$, define the parabolic subgroup $P^{\alpha} \subseteq G L(n, \mathbf{C})$ by

$$
P^{\alpha}=\left\{g \in G L(n, \mathbf{C}) \mid g E^{\alpha}(i)=E^{\alpha}(i) \forall i\right\}
$$

Then the compatibility condition says precisely that for all cones $\sigma \in \Sigma$,

$$
\bigcap_{\alpha \in|\sigma|} P^{\alpha} \text { contains a maximal torus of } G L(n, \mathbf{C})
$$

Note that the bundle compatibility conditions are triangulation-dependent; they are sensitive to more than just the edges of the fan. In particular, a set of filtrations that defines a bundle in one triangulation may fail to define a bundle after a flop (or other birational transformation on the toric variety preserving the edges of the fan). In such a case, instead of defining a bundle in the flopped triangulation, we would only get a reflexive sheaf.

Specifying a parabolic subgroup, as in equation (3), does not uniquely specify the filtration - the indices at which the filtration changes dimension also are meaningful. We will see later (section 4.4) that this information corresponds to a choice of ample line bundle on the (partial) flag manifold $G / P^{\alpha}$, in addition to the choice of parabolic subgroup $P^{\alpha}$.

An equivariant vector bundle is defined uniquely by a set of filtrations (one for each toric divisor) satisfying the compatibility condition above, up to a simultaneous rotation of all filtrations by an element of $G L(n, \mathbf{C})$.

For example, let's discuss how line bundles are described in this language. Let $D=\sum a_{\alpha} D_{\alpha}$ be a Cartier divisor on the toric variety, then the filtration on divisor $D_{\alpha}$ is given by

$$
E^{\alpha}(i)= \begin{cases}\mathbf{C} & i \leq a_{\alpha} \\ 0 & i>a_{\alpha}\end{cases}
$$


(Recall that for line bundles the choice of equivariant structure amounts to simply being specific about the choice of divisor.) At this point we can see the necessity of the smoothness condition. On a singular toric variety, not all divisors are Cartier, i.e., not all divisors $D=\sum a_{\alpha} D_{\alpha}$ define line bundles. Equivariant sheaves on singular toric varieties will be discussed in section 4.5.1.

For another example, consider the direct sum of two line bundles $\mathcal{O}\left(D_{1}\right) \oplus$ $\mathcal{O}\left(D_{2}\right)$. Write $D_{1}=\sum a_{1 \alpha} D_{\alpha}, D_{2}=\sum a_{2 \alpha} D_{\alpha}$ (where the $D_{\alpha}$ are the toric divisors), then

$$
E^{\alpha}(i)= \begin{cases}\mathbf{C}^{2} & i \leq \min \left(a_{1 \alpha}, a_{2 \alpha}\right) \\ \mathbf{C} & \min \left(a_{1 \alpha}, a_{2 \alpha}\right)<i \leq \max \left(a_{1 \alpha}, a_{2 \alpha}\right) \\ 0 & i>\max \left(a_{1 \alpha}, a_{2 \alpha}\right)\end{cases}
$$

In particular, a vector bundle splits globally into a direct sum of holomorphic line bundles precisely when all the filtrations (not just associated to toric divisors in any one cone) satisfy the compatibility condition (4).

It may naively appear that all information is contained in the values of $i$ at which a filtration changes dimension, but this is in fact false. We will see later that the precise vector subspaces appearing also carry information - for example, they partly determine most of the Chern classes.

Note that we can rederive the (weaker) equivariant version of a famous theorem of Grothendieck, which says that all holomorphic vector bundles on $\mathbf{P}^{1}$ split into a direct sum of holomorphic line bundles. Since any two parabolic subgroups intersect in a maximal torus (at least), and as $\mathbf{P}^{1}$ has only two toric divisors, it should be obvious that all equivariant vector bundles on $\mathbf{P}^{1}$ split as indicated.

\subsection{Kaneyama's Approach}

There is a closely related approach to equivariant bundles on toric varieties described in $[6,7]$. This approach is more intuitively clear than Klyachko's, but computationally far more cumbersome.

The basic idea behind Kaneyama's approach is to study equivariant vector bundles on each element of a cover of the base space. In particular, the maximal cones defining coordinate charts on the toric variety provide a suitable cover. On each element of the cover (affine spaces, by the assumption of smoothness), the vector bundle trivializes into a sum of one-dimensional 
representations of the algebraic torus $T$ defining the toric variety. Put another way, for each maximal cone of the toric variety, for a rank $r$ vector bundle $\mathcal{E}$ we can associate $r$ characters of the algebraic torus (elements of the lattice $M$, in the notation of [8]). This decomposition of the vector space $\mathbf{C}^{r}$ describes precisely how the vector space transforms (equivariantly) under the action of the algebraic torus.

Given this set of $r$ characters associated with some maximal cone, we can now associate a set of integers with each toric divisor in the maximal cone. (These integers are half the data Kaneyama needs to describe a vector bundle.) If we label the characters as $\chi_{1}, \ldots \chi_{r}$, then the integers associated with toric divisor $\alpha$ are precisely $\left\langle\chi_{1}, \alpha\right\rangle, \ldots\left\langle\chi_{r}, \alpha\right\rangle$.

In fact, this is precisely a generalization of the description of line bundles in [8]. There, a line bundle on a compact toric variety was specified by associating a character ${ }^{10}$ to each maximal cone. The characters on distinct cones need not agree, rather they can differ by a character perpendicular to all vectors in the intersection of the cones. Thus, the characters themselves are not well-defined. However, it is possible to associate well-defined integers with each toric divisor $\alpha$, as $\langle\chi, \alpha\rangle$ where $\chi$ is a character associated with a maximal cone containing $\alpha$. In Kaneyama's generalization, we associate $r$ characters with each maximal cone, and an element of $G L(r, \mathbf{C})$ with each overlap of cones. The characters themselves need not be well-defined; however the integers $\left\langle\chi_{1}, \alpha\right\rangle,\left\langle\chi_{2}, \alpha\right\rangle, \ldots$ are well-defined.

These integers can also be derived from Klyachko's filtration description. Let $E^{\alpha}(i)$ be a set of filtrations associated with the bundle $\mathcal{E}$, then define

$$
E^{[\alpha]}(i)=\frac{E^{\alpha}(i)}{E^{\alpha}(i+1)}
$$

(recall that $\left.E^{\alpha}(i+1) \subseteq E^{\alpha}(i)\right)$. Given this definition, the integers Kaneyama associates with a toric divisor $\alpha$ are precisely the $i$ for which $E^{[\alpha]}(i)$ is nonzero, counted with multiplicity equal to $\operatorname{dim} E^{[\alpha]}(i)$.

An example should make this more clear. Suppose we have a filtration defined by

$$
E^{\alpha}(i)= \begin{cases}\mathbf{C}^{3} & i \leq 5 \\ \mathbf{C}^{2} & i=6,7 \\ 0 & i>7\end{cases}
$$

\footnotetext{
${ }^{10}$ Technical fiends will note that each character is the location of the generator of the principal fractional ideal associated to that cone.
} 
then

$$
E^{[\alpha]}(i)= \begin{cases}\mathbf{C} & i=5 \\ \mathbf{C}^{2} & i=7 \\ 0 & \text { otherwise }\end{cases}
$$

then in Kaneyama's description, to the toric divisor $\alpha$ we associate the integers 5,7 , and 7 .

In fact, $\operatorname{dim} E^{[\alpha]}(i)$ is counting the multiplicities with which characters of the algebraic torus $T$ are appearing in maximal cones containing $\alpha$. The multiplicity with which a character $\chi$ appears is equal to $\operatorname{dim} E^{[\alpha]}(\langle\chi, \alpha\rangle)$.

So far we have given only half of Kaneyama's description of vector bundles. To each toric divisor we have described how to associate a set of integers, and shown how this is related to Klyachko's description of vector bundles. However, these integers are not sufficient to completely describe the bundle, as we also need to describe the transition functions on overlaps of coordinate charts.

In Kaneyama's description the transition functions for a rank $r$ bundle are given as elements of $G L(r, \mathbf{C})$ assigned to all pairs of cones, satisfying the usual compatibility conditions. How can such transition functions be derived from Klyachko's description? As was shown in [2, p. 344], if $\mathcal{E}, \mathcal{F}$ are equivariant bundles on an affine variety, then the space of toral homomorphisms $\operatorname{Hom}_{T}(\mathcal{E}, \mathcal{F})$ is isomorphic to the space of linear operators $\phi: E \rightarrow F$ compatible with the filtrations as $\phi\left(E^{\alpha}(i)\right) \subseteq F^{\alpha}(i) \forall \alpha, i$. In particular, on overlaps between cones $\sigma_{1}, \sigma_{2}$, transition functions must map each filtration associated to divisor $\alpha \in\left|\sigma_{1} \cap \sigma_{2}\right|$ back into itself. Thus, it should be clear that for any cone $\sigma$, Kaneyama's transition function must be an element of

$$
\bigcap_{\alpha \in|\sigma|} P^{\alpha}
$$

Conversely, given the data Kaneyama specifies it is possible to recreate Klyachko's description.

Note in particular that both descriptions implicitly rely on equivariance of the bundles in question. If a bundle is not equivariant, then over any coordinate chart it trivializes and so of course can be written as a direct sum of characters, but there need no longer be any relation between characters of distinct coordinate charts, and so one can no longer associate an invariant set of integers with each toric divisor. Similarly, Klyachko's description fails, as there is no longer an invariant way to associate a filtration with each toric divisor. 


\subsection{Applications}

Here we will quote some results presented in $[1,2,3,4]$ on Chern classes and sheaf cohomology groups of equivariant bundles over toric varieties. We will also closely follow the notation of these references, in order to make the connection more clear to the reader.

\subsubsection{Chern Classes}

Before describing the Chern classes, we will first describe a natural resolution of any bundle $\mathcal{E}$ by direct sums of line bundles, as described in [2]. Given this resolution, computing the Chern classes will then be quite straightforward.

Schematically, this resolution is an exact sequence

$0 \rightarrow \mathcal{E} \rightarrow \bigoplus_{\text {codim } \sigma=0} \sigma \otimes \mathcal{E} \rightarrow \bigoplus_{\text {codim } \sigma=1} \sigma \otimes \mathcal{E} \rightarrow \cdots \rightarrow \underset{\text { codim } \sigma=n-1}{\bigoplus} \sigma \otimes \mathcal{E} \rightarrow \emptyset \otimes \mathcal{E} \rightarrow 0$

where each $\sigma \otimes \mathcal{E}$ is a holomorphic bundle, of the same rank as $\mathcal{E}$, defined by the filtrations ${ }^{11}$

$$
(\sigma \otimes \mathcal{E})^{\alpha}(i)= \begin{cases}E^{\alpha}(i) & \alpha \in|\sigma| \\ E & \alpha \notin|\sigma|, i \leq f(\alpha) \\ 0 & \alpha \notin|\sigma|, i>f(\alpha)\end{cases}
$$

and where $f(\alpha)$ is a function $f: D_{\alpha} \rightarrow \mathbf{Z}$ that assigns to each toric divisor an integer, the largest at which the filtration is nonzero. In other words, $E^{\alpha}(i)=0$ precisely when $i>f(\alpha)$. (We will see that the function $f(\alpha)$ cancels out of Chern class computations.) Note that the notation used above is rather poor - nothing is being tensored in $\sigma \otimes \mathcal{E}$, rather $\sigma \otimes \mathcal{E}$ merely denotes an auxiliary bundle associated to cone $\sigma$. Each map in the exact sequence (5) is the obvious inclusion between filtrations.

Each bundle $\sigma \otimes \mathcal{E}$ splits into a sum of line bundles, as we now show. By assumption, the filtrations on the toric divisors of any single cone $\sigma$ are compatible, in the sense of equation (4), and as the filtrations on all other toric divisors are trivial, all the filtrations are compatible, so the bundle splits globally.

Note that the line bundles into which $\sigma \otimes \mathcal{E}$ splits are in one-to-one correspondence with generators of $E$. For example, suppose $\mathcal{E}$ is rank two,

\footnotetext{
${ }^{11}$ The equation shown corrects typos in equation (3.3) of [2].
} 
defined on a toric surface. Suppose for divisors we shall label 1,2,

$$
\operatorname{dim} E^{[\alpha]}(i)= \begin{cases}1 & i=0 \\ 1 & i=1 \\ 0 & i \neq 0,1\end{cases}
$$

Then if the two one-dimensional vector spaces at $i=1$ coincide as subspaces of $\mathbf{C}^{2}, c(\sigma \otimes \mathcal{E})=\left[1+D_{1}+D_{2}\right]$. Otherwise, if those two one-dimensional vector spaces are generic, we have $c(\sigma \otimes \mathcal{E})=\left[1+D_{1}\right]\left[1+D_{2}\right]$. More generally, although it will turn out that the first Chern class depends only on the dimensions of each element of each filtration, to determine the higher Chern classes more information about the filtrations is required.

Before using the resolution above to derive the Chern classes of $\mathcal{E}$, we shall define some notation. Let $\mathcal{E}$ be an equivariant bundle on a toric variety $X$ with fan $\Sigma$, and let $E^{\alpha}(i)$ be a family of compatible filtrations defining $\mathcal{E}$. Define

$$
\begin{gathered}
E^{\sigma}(\chi)=\bigcap_{\alpha \in|\sigma|} E^{\alpha}(\langle\chi, \alpha\rangle) \\
E^{[\sigma]}(\chi)=\frac{E^{\sigma}(\chi)}{\sum_{i_{\alpha}} \bigcap_{\alpha \in|\sigma|} E^{\alpha}\left(i_{\alpha}\right)}
\end{gathered}
$$

where in equation (7) the sum is taken over all $i_{\alpha} \in \mathbf{Z}$, and the intersection is over $\alpha \in|\sigma|$ such that $i_{\alpha} \geq\langle\chi, \alpha\rangle$ and for at least one $\left.\alpha \in|\sigma|, i_{\alpha}\right\rangle\langle\chi, \alpha\rangle$. In particular, this implies

$$
E^{[\alpha]}(i)=\frac{E^{\alpha}(i)}{E^{\alpha}(i+1)}
$$

In fact, $E^{\sigma}$ is a freely-generated $\mathbf{C}\left[\sigma^{\vee}\right]$ module (see section 4.5.1), and $E^{[\sigma]}$ locates its generators.

Now, let us derive the Chern classes of $\mathcal{E}$, using the resolution (5). First consider the bundle $\alpha \otimes \mathcal{E}$, where $\alpha$ is an edge of the fan. The first Chern class is clearly

$$
c_{1}(\alpha \otimes \mathcal{E})=\sum_{i} i \operatorname{dim} E^{[\alpha]}(i) D_{\alpha}+\sum_{\alpha^{\prime} \neq \alpha} f\left(\alpha^{\prime}\right)(\operatorname{dim} E) D_{\alpha^{\prime}}
$$

Using the splitting principle, we can derive the total Chern class of $\alpha \otimes \mathcal{E}$ :

$$
c(\alpha \otimes \mathcal{E})=\prod_{\chi \in \hat{T}_{\alpha}}\left[1+\sum_{\alpha^{\prime} \in|\Sigma|} f_{\chi}^{\alpha}\left(\alpha^{\prime}\right) D_{\alpha^{\prime}}\right]^{\operatorname{dim} E^{[\alpha]}(\chi)}
$$


where

$$
f_{\chi}^{\sigma}(\alpha)= \begin{cases}f(\alpha) & \alpha \notin|\sigma| \\ \langle\chi, \alpha\rangle & \alpha \in|\sigma|\end{cases}
$$

and where $\hat{T}_{\sigma}$ is the weight lattice $M=\operatorname{Hom}\left(T, \mathbf{C}^{\times}\right)$modulo the sublattice $\sigma^{\perp} \cap M$. (More formally, define $T_{\sigma}$ to be the subgroup of $T$ stabilizing the subvariety corresponding to $\sigma$ (the subgroup of $T$ whose Lie algebra is generated by $\sigma$ and $-\sigma)$ then $\hat{T}_{\sigma}=$ Hom $\left(T_{\sigma}, \mathbf{C}^{\times}\right)$.) In other words, if we took the product over $\chi \in M$ rather than just $\chi \in \hat{T}_{\alpha}$, we would overcount.

It should now be clear that in general,

$$
c(\sigma \otimes \mathcal{E})=\prod_{\chi \in \hat{T}_{\sigma}}\left[1+\sum_{\alpha \in|\Sigma|} f_{\chi}^{\sigma}(\alpha) D_{\alpha}\right]^{\operatorname{dim} E^{[\sigma]}(\chi)}
$$

Now, we should get the same result for $c(\mathcal{E})$ if we increase each value of $f: D_{\alpha} \rightarrow \mathbf{Z}$, so the expression for $c(\mathcal{E})$ must be independent of the $f(\alpha)$ 's, so we can simply set them to zero and immediately recover the result

$$
c(\mathcal{E})=\prod_{\sigma \in \Sigma, \chi \in \hat{T}_{\sigma}}\left(1+\sum_{\alpha \in|\sigma|}\langle\chi, \alpha\rangle D_{\alpha}\right)^{(-)^{\operatorname{codim} \sigma} \operatorname{dim} E^{[\sigma]}(\chi)}
$$

(If the reasoning behind our omission of the $f(\alpha)$ 's seemed too loose, the reader is encouraged to check a few examples in detail.) Similarly, one can calculate the Chern character of $\mathcal{E}$ to be

$$
\operatorname{ch}(\mathcal{E})=\sum_{\sigma \in \Sigma, \chi \in \hat{T}_{\sigma}}(-)^{\operatorname{codim} \sigma} \operatorname{dim} E^{[\sigma]}(\chi) \exp \left(\sum_{\alpha \in|\sigma|}\langle\chi, \alpha\rangle D_{\alpha}\right)
$$

where $D_{\alpha}$ denotes the toric divisor associated with $\alpha$. Thus, for example,

$$
c_{1}(\mathcal{E})=\sum_{i \in \mathbf{Z}, \alpha \in|\Sigma|} i \operatorname{dim} E^{[\alpha]}(i) D_{\alpha}
$$

\subsubsection{Sheaf Cohomology Groups}

It is also straightforward to calculate sheaf cohomology groups $H^{p}(X, \mathcal{E})$. Since $\mathcal{E}$ is equivariant, the sheaf cohomology groups can be decomposed into groups each associated with an irreducible representation of the algebraic torus $^{12}$ :

$$
H^{p}(X, \mathcal{E})=\oplus_{\chi} H^{p}(X, \mathcal{E})_{\chi}
$$

\footnotetext{
${ }^{12}$ If $\mathcal{E}$ is not equivariant, then the sheaf cohomology groups have no such decomposition.
} 
In particular, all irreducible representations of the algebraic torus are onedimensional, given precisely by the characters $\chi$, and this decomposition of the sheaf cohomology is known as an isotypic decomposition. (Note that each $H^{p}(X, \mathcal{E})_{\chi}$ need not itself be one-dimensional; it is merely naturally associated with a (one-dimensional) irreducible representation.)

To calculate isotypic components of the sheaf cohomology groups $H^{p}(X, \mathcal{E})$ we use the complex ${ }^{13} C^{*}(\mathcal{E}, \chi)$ :

$$
0 \rightarrow \bigoplus_{\operatorname{dim} \sigma=n} E^{\sigma}(\chi) \rightarrow \cdots \rightarrow \bigoplus_{\operatorname{dim} \sigma=2} E^{\sigma}(\chi) \rightarrow \bigoplus_{\operatorname{dim} \sigma=1} E^{\sigma}(\chi) \rightarrow E \rightarrow 0
$$

where $n=\operatorname{dim} X, E$ is the generic fiber of the vector bundle. We will observe later (section 4.5.1) that $E^{\sigma}$ is a graded $\mathbf{C}\left[\sigma^{\vee}\right]$-module, equal to $\Gamma\left(U_{\sigma}, \mathcal{E}\right)$ (where $U_{\sigma}$ is the open set in $X$ determined by cone $\sigma$ ), so this is precisely a Cech cohomology calculation, and the differential is then defined in the usual fashion. Then,

$$
H^{p}(X, \mathcal{E})_{\chi}=H^{p}\left(C^{*}(\mathcal{E}, \chi)\right)
$$

It should be clear that

$$
\sum_{i}(-)^{i} \operatorname{dim} H^{i}(X, \mathcal{E})_{\chi}=\sum_{\sigma \in \Sigma}(-)^{\operatorname{codim} \sigma} \operatorname{dim} E^{\sigma}(\chi)
$$

and also ${ }^{14}$

$$
H^{0}(X, \mathcal{E})_{\chi}=\bigcap_{\alpha \in|\Sigma|} E^{\alpha}(\chi)
$$

In principle, calculating the sheaf cohomology of the restriction of $\mathcal{E}$ to a hypersurface in the toric variety is now straightforward. Suppose our hypersurface is specified by some divisor $\mathrm{D}$, then we have an exact sequence

$$
0 \rightarrow \mathcal{E}(-D) \rightarrow \mathcal{E} \rightarrow \mathcal{E} \otimes \mathcal{O}_{D} \rightarrow 0
$$

where $\mathcal{O}_{D}$ is a skyscraper sheaf with support on $D$. From this short exact sequence we can derive a long exact sequence describing sheaf cohomology of $\mathcal{E}$ restricted to the hypersurface in terms of sheaf cohomology on the toric variety. Since $\mathcal{E}$ is equivariant, $\mathcal{E}(-D)$ is also equivariant, so in principle this calculation can be performed via the above technology.

\footnotetext{
${ }^{13}$ In references $[1,2,3,4]$, this complex is ordered in the opposite direction and denoted $C_{*}(\mathcal{E}, \chi)$. We have chosen different conventions for clarity.

${ }^{14}$ The next result holds only for reflexive, not for more general torsion-free sheaves.
} 


\subsection{Alternate Gauge Groups}

So far in this paper we have discussed equivariant holomorphic vector bundles whose structure group is $G L(n, \mathbf{C})$. In principle, however, principal bundles with other (algebraic) gauge groups can be discussed equally easily in Klyachko's language, as was noted in [4].

When discussing $G L(n, \mathbf{C})$ bundles, we can speak in terms either of filtrations of a vector space or in terms of parabolic subgroups of $G L(n, \mathbf{C})$ which preserve the filtration (as in equation (3)) (paired with ample line bundles, as mentioned in section 4.1). For more general structure groups $G$ there is no filtration description, but the description in terms of parabolics still holds.

In other words, to discuss principal bundles with arbitrary structure group $G$, one associates a parabolic subgroup $P^{\alpha} \subset G$ to each toric divisor $\alpha$, together with an ample line bundle $L_{\alpha}$ on $G / P^{\alpha}$.

The compatibility condition on the parabolic subgroups associated to each toric divisor is very nearly the same as equation (4). Rather than demand the parabolics intersect in a maximal torus of $G L(n, \mathbf{C})$, one demands the parabolics intersect in a maximal torus of $G$. In other words, for all cones $\sigma \in \Sigma$, if $P^{\alpha}$ denotes the parabolic associated with edge $\alpha$, then

$$
\bigcap_{\alpha \in|\sigma|} P^{\alpha} \text { contains a maximal torus of } G
$$

Now, let us consider the ample line bundles $L_{\alpha}$ paired with the parabolics $P^{\alpha}$ more closely. First, we shall describe a construction of vector bundles on flag manifolds $G / P^{\alpha}$, then we shall describe how the ample line bundles are derived for $G L(r, \mathbf{C})$ bundles.

There is a natural way to construct vector bundles on $G / P$. First, note that $G \rightarrow G / P$ is a principal $P$-bundle over $G / P$. Then, there exists a family of vector bundles over $G / P$ (associated to the principal $P$-bundle $G \rightarrow G / P$ ), corresponding to representations of $P$. In other words, given a representation of $P$, consisting of a vector space $V$ and a $P$-action $\lambda: P \times V \rightarrow V$, we can construct a vector bundle associated to $G \rightarrow G / P$. The total space of this vector bundle is just $G \times{ }_{P} V$. In particular, line bundles constructed in this fashion automatically come with a canonical $G$-linearization (in fact, if $G$ is semisimple then the $G$-linearization is unique). (This is the setup of the Bott-Borel-Weil theorem [103], which states - among other things - that the sections of an ample line bundle associated to $G \rightarrow G / P$ form an irreducible representation of $G$.) 
Now, we shall describe how to construct the ample line bundles $L_{\alpha}$ for $G=G L(r, \mathbf{C})$ bundles. First, associate $r=$ rank $\mathcal{E}$ integers to the filtration $E^{\alpha}$. These integers are the integers $i$ such that $E^{[\alpha]}(i)$ is nonzero, counted with multiplicity equal to $\operatorname{dim} E^{[\alpha]}(i)$. (In other words, these are the same integers that Kaneyama associates with a toric divisor, as described in section 4.2.) These integers define a point in the weight lattice of $G$. If we put them in increasing order, then they correspond to a weight in the fundamental Weyl chamber, and so describe an ample line bundle. Denote this line bundle on $G / P^{\alpha}$ by $L_{\alpha}$. As promised in section 4.1, the ample line bundles $L_{\alpha}$ encode information about the filtrations that is missed by only specifying parabolics.

At this point we should mention an interesting subtlety. Irreducible representations of a parabolic $P$ are in one-to-one correspondence with irreducible representations of a Levi factor of the parabolic, so when the parabolic is a Borel subgroup, any Levi factor is a product of $\mathbf{C}^{\times}$'s, and so any set of integers defines the dominant weight of a one-dimensional irreducible representation of $P$ - and in particular defines a line bundle on $G / P$. When $P$ is not a Borel, however, not any set of integers will be the dominant weight for a one-dimensional irreducible representation of $P$. In such a case how can we be guaranteed of getting a line bundle on $G / P$ (as opposed to some higher rank vector bundle) in the prescription above? For simplicity, consider the case that only two of the integers associated to divisor $\alpha$ coincide. Then, a Levi factor of the corresponding parabolic will be the product of several $\mathbf{C}^{\times}$'s with a $G L(2, \mathbf{C})$. The two coincident integers define a weight of $G L(2, \mathbf{C})$. However, since the integers are identical, the semisimple part of $G L(2, \mathbf{C})$, namely $S L(2, \mathbf{C})$, acts trivially on the representation defined. The only part of $G L(2, \mathbf{C})$ that acts nontrivially is the overall $\mathbf{C}^{\times}$factor, and so we clearly have defined a one-dimensional irreducible representation of $P$. It should be clear in general that the prescription above will always yield one-dimensional irreducible representations of $P$, and in particular line bundles on $G / P$.

Principal $G$-bundles are uniquely identified by a set of parabolics obeying the compatibility condition (10), together with a set of ample line bundles, up to an overall simultaneous $G$ rotation of the parabolics.

The reader may wonder why vector bundles with structure group $G$ do not have a description in terms of filtrations analogous to that discussed earlier. Given some vector space $V$ acted on by a representation of $G$ inside some $G L(n, \mathbf{C})$, we can certainly associate parabolic subgroups of $G L(n, \mathbf{C})$ to filtrations, and then we could intersect those parabolic subgroups of $G L(n, \mathbf{C})$ with the image of $G$. Unfortunately those intersections need not be parabolic 
subgroups, and in fact in general to describe a vector bundle with structure group $G$ in this fashion takes a great deal of work, dependent upon both $G$ and the representation chosen. We shall not examine such constructions in detail in this paper.

\subsection{Equivariant Torsion-free Sheaves}

In this section we will discuss torsion-free sheaves on arbitrary toric varieties. (Note that our previous discussions of bundles and reflexive sheaves were limited to smooth varieties). In section 4.5.1 we discuss the basic principles on which our description is based. In section 4.5.2 we discuss torsion-free sheaves on smooth toric varieties. In section 4.5.3 we discuss how global Ext groups can be calculated in principle for equivariant sheaves on toric varieties. In section 4.5.4 we discuss torsion-free sheaves on the singular toric variety, $\mathbf{P}_{1,1,2}^{2}$. Torsion-free sheaves are rather more complicated to discuss on singular toric varieties, but this two example should sufficiently illustrate the general method. Finally, in section 4.5 .5 we discuss the distinction between Cartier and Weil divisors, and how it can be seen explicitly in this framework.

Much of section 4.5.2 was originally discussed in [4], however the rest of the material in this section is new.

\subsubsection{Basic Principles}

In principle, coherent sheaves can be constructed over varieties by associating to each element Spec $R_{i}$ of an affine cover a finite-rank $R_{i}$-module $M_{i}$, in a "mutually compatible" way (see [107, section II.5]). Compatibility is defined by associating a module to every intersection of the affine cover, together with restriction maps. In particular, to build a coherent sheaf on a toric variety, one must associate a finite-rank module to each cone.

When the coherent sheaf is equivariant, we can refine the description further. The module associated to each cone $\sigma$ has a weight space decomposition under the algebraic torus $T$ whose compactification is the toric variety. For an $n$-dimensional toric variety, each module is $M$-graded, where $M \cong \mathbf{Z}^{n}$ is the weight lattice of $T$. (This is essentially an isotypic decomposition of the module under the action of the algebraic torus, which holds only for the modules appearing in equivariant sheaves.) Each (isotypic) component of 
the module is a vector space ${ }^{15}$, and in fact we shall see each component is a vector subspace of some fixed vector space. We shall denote the component of a module $E$ associated to character $\chi$ by $E(\chi)$ :

If the module is torsion-free ${ }^{16}$ then the action of the underlying monoid algebra $\mathbf{C}\left[\sigma^{\vee}\right]$ induces inclusion maps between the graded components of the module. Let us work through this a little more carefully. Let $E^{\sigma}$ denote the module associated to a cone $\sigma$, then for $\mu \in \sigma^{\vee}$ and for all $\chi \in M$ we have maps $E^{\sigma}(\chi) \rightarrow E^{\sigma}(\chi+\mu)$. Since the module is torsion-free, these maps are injective. In fact, we can identify spaces with their images, and regard these maps as inclusions.

In passing, note that for any $\rho$ in the interior of $\sigma^{\vee}$, it is true that for all components of $E^{\sigma}$,

$$
E^{\sigma}(\chi) \subseteq \lim _{n \rightarrow \infty} E^{\sigma}(n \rho)
$$

This is because for all $\chi \in M$, there exists $N \in \mathbf{N}$ such that $\langle\chi+N \rho, \nu\rangle>0$ for all $\nu \in \sigma^{\vee}$, i.e., $\chi+N \rho \in \sigma^{\vee}$. In particular, this means that each component $E^{\sigma}(\chi)$ is a subset of some fixed vector space, and in fact this vector space is independent of $\sigma$, as expected.

To clarify these remarks, consider the case that the affine space is of the form

$$
\text { Spec } \mathbf{C}\left[x_{1}^{a_{11}} x_{2}^{a_{21}} \cdots x_{n}^{a_{n 1}}, \cdots, x_{1}^{a_{1 k}} x_{2}^{a_{2 k}} \cdots x_{n}^{a_{n k}}\right]
$$

then the inclusions are

$$
\begin{aligned}
E^{\sigma}\left(i_{1}, \cdots, i_{n}\right) & \hookrightarrow E^{\sigma}\left(i_{1}+a_{11}, i_{2}+a_{21}, \cdots, i_{n}+a_{n 1}\right) \\
& \hookrightarrow E^{\sigma}\left(i_{1}+a_{12}, i_{2}+a_{22}, \cdots, i_{n}+a_{n 2}\right) \\
& \cdots \\
& \hookrightarrow E^{\sigma}\left(i_{1}+a_{1 k}, i_{2}+a_{2 k}, \cdots, i_{n}+a_{n k}\right)
\end{aligned}
$$

In order to recover decreasing filtrations on smooth spaces, the conventions used earlier in this paper, merely work on the smooth space

$$
\text { Spec } \mathbf{C}\left[x_{1}^{-1}, x_{2}^{-1}, \cdots, x_{n}^{-1}\right]
$$

\footnotetext{
${ }^{15}$ Each isotypic component of an $A$-module $M$, where both $A$ and $M$ have $T$ actions compatible with the ring action, is a module over the weight zero part of $A$. In the cases relevant here, the weight zero part of the ring will always be the field $\mathbf{C}$. A module over a field is precisely a vector space, thus each isotypic component of the module is a vector space.

${ }^{16}$ Usually one states a sheaf is torsion-free when each stalk is torsion-free, meaning, when each localization of the module is torsion-free. However, over the rings we shall encounter in toric varieties, torsion-free is a local property, in the sense that if it is true for all localizations of a module, then it is true for the entire module. (See for example [84, exercise 3.13].) The same statement is also true of reflexivity and local freedom.
} 
To make this perspective more clear, let us consider some examples. The structure sheaf on $\mathbf{C}^{2}=\operatorname{Spec} \mathbf{C}\left[x_{1}, x_{2}\right]$ is equivariant, and has bigraded module precisely $\mathbf{C}\left[x_{1}, x_{2}\right]$, meaning

$$
E\left(i_{1}, i_{2}\right)= \begin{cases}\mathbf{C} & i_{1} \geq 0 \text { and } i_{2} \geq 0 \\ 0 & \text { otherwise }\end{cases}
$$

for the trivial choice of equivariant structure. Now, consider an ideal ${ }^{17}$ sheaf on $\mathbf{C}^{2}=$ Spec $\mathbf{C}\left[x_{1}, x_{2}\right]$. In fact, consider the rank 1 ideal sheaf vanishing to order 1 at $x_{1}=x_{2}=0$. The module defining the sheaf is precisely the ideal with generators $\left(x_{1}, x_{2}\right)$ inside $\mathbf{C}\left[x_{1}, x_{2}\right]$. This ideal sheaf is equivariant ${ }^{18}$, and we have the bigraded module

$$
E\left(i_{1}, i_{2}\right)= \begin{cases}\mathbf{C} & i_{1}>0 \text { and } i_{2} \geq 0 \\ \mathbf{C} & i_{1} \geq 0 \text { and } i_{2}>0 \\ 0 & \text { otherwise }\end{cases}
$$

As expected, the ideal sheaf sits naturally inside the structure sheaf.

Now, consider the case that a cone $\sigma$ is not maximal, for example, that Spec $\mathbf{C}\left[\sigma^{\vee}\right]=\mathbf{C} \times \mathbf{C}^{\times}$. In such an example, we have inclusion maps of the form

$$
\begin{aligned}
E^{\sigma}\left(i_{1}, i_{2}\right) & \hookrightarrow E^{\sigma}\left(i_{1}+1, i_{2}\right) \\
& \hookrightarrow E^{\sigma}\left(i_{1}, i_{2}+1\right) \\
& \hookrightarrow E^{\sigma}\left(i_{1}, i_{2}-1\right)
\end{aligned}
$$

Clearly, $E^{\sigma}\left(i_{1}, i_{2}\right)$ is independent of $i_{2}$. A more nearly invariant way to say this is that the module associated to a cone $\sigma$ is nontrivial only over the lattice $\hat{T}_{\sigma}$, where $\hat{T}_{\sigma}$ is simply the weight lattice $M$ of the algebraic group modulo the subgroup $\sigma^{\perp}$ - in other words, $E^{\sigma}$ is constant along directions in $\sigma^{\perp}$.

So far we have described modules associated to any individual cone; how are modules over distinct cones related? If $\tau$ is a subcone of $\sigma$, then by definition $^{19}$

$$
E^{\tau}=E^{\sigma} \otimes_{\mathbf{C}\left[\sigma^{\vee}\right]} \mathbf{C}\left[\tau^{\vee}\right]
$$

We can show that if $\tau$ is a subcone of $\sigma$ and $\rho$ is in the interior of $\tau^{\perp} \cap \sigma^{\vee}$, then

$$
E^{\tau}(\chi)=\lim _{n \rightarrow \infty} E^{\sigma}(\chi+n \rho)
$$

\footnotetext{
${ }^{17}$ For an introduction to ideal sheaves see the first appendix of [13].

${ }^{18}$ Technical fiends will note that we can define equivariant Hilbert schemes.

${ }^{19} E^{\tau}$ is simply the restriction of the module $E^{\sigma}$ associated to affine space $\operatorname{Spec} \mathbf{C}\left[\sigma^{\vee}\right]$ to $\operatorname{Spec} \mathbf{C}\left[\tau^{\vee}\right] \subset \operatorname{Spec} \mathbf{C}\left[\sigma^{\vee}\right]$.
} 
for all $\chi \in M$. (In particular, this shows the maximal vector space $E$ is independent of $\sigma$, as claimed earlier.) The point is to show that for all $\mu \in \tau^{\vee}$, there exists $N \in \mathrm{N}$ such that $\mu+N \rho \in \sigma^{\vee}$. This follows from the definition of $\rho$ : for any fixed $\mu \in \tau^{\vee},\langle\mu, \nu\rangle$ is bounded from below as we vary $\nu \in \sigma^{\vee}$, so as $\langle\rho, \nu\rangle \geq 0$ for all $\nu \in \sigma^{\vee}$, there exists $N \in \mathbf{N}$ such that

$$
N\langle\rho, \nu\rangle+\langle\mu, \nu\rangle \geq 0
$$

for all $\nu \in \sigma^{\vee}$. Thus, for any $\mu \in \tau^{\vee}$, there exists $N \in \mathbf{N}$ such that $N \rho+\mu \in \sigma^{\vee}$, and so we have an inclusion map

$$
E^{\sigma}(\chi-\mu) \hookrightarrow E^{\sigma}(\chi+N \rho)
$$

for all $\chi \in M$, so in particular we find

$$
E^{\tau}(\chi)=\lim _{n \rightarrow \infty} E^{\sigma}(\chi+n \rho)
$$

We can summarize these results as follows. Suppose $\tau, \sigma$ are both cones, and $\tau \subset \sigma$. For any $\chi \in \hat{T}_{\sigma}$, we have the inclusion

$$
E^{\sigma}(\chi) \subseteq E^{\tau}(\chi)
$$

where on the right side of the equation above we can interpret $\chi$ as an element of $\hat{T}_{\tau}$, using the natural projection $\hat{T}_{\sigma} \rightarrow \hat{T}_{\tau}$. Moreover, for any $\chi \in \hat{T}_{\tau}$,

$$
E^{\tau}(\chi)=\bigcup_{\widetilde{\chi}} E^{\sigma}(\widetilde{\chi})
$$

where the union is over $\tilde{\chi}$ that project to $\chi$ under $\hat{T}_{\sigma} \rightarrow \hat{T}_{\tau}$.

In the special case that a sheaf is reflexive, this description can be simplified. The module $E^{\sigma}$ associated to any cone $\sigma$ is the intersection of the modules associated to the toric divisors in the cone:

$$
E^{\sigma}(\chi)=\bigcap_{\alpha \in|\sigma|} E^{\alpha}(\chi)
$$

(This result is stated without proof in [4] for the special case of smooth varieties.) In fact, somewhat more generally, for any equivariant torsion-free sheaf with modules $\left\{E^{\sigma}\right\}$, its bidual has modules

$$
\left(E^{\vee \vee}\right)^{\sigma}(\chi)=\bigcap_{\alpha \in|\sigma|} E^{\alpha}(\chi)
$$

The reader should find this result intuitively reasonable, for the following reason. For any torsion-free sheaf $\mathcal{E}$, there is an inclusion $\mathcal{E} \rightarrow \mathcal{E}^{\vee \vee}$. In other 
words, any torsion-free sheaf should naturally map into its bidual. Now, for any set of modules $\left\{E^{\sigma}\right\}$, it is always true that

$$
E^{\sigma}(\chi) \subseteq \bigcap_{\alpha \in|\sigma|} E^{\alpha}(\chi)
$$

so the expression given for $\left(E^{\vee \vee}\right)^{\sigma}$ should seem extremely natural.

We shall now demonstrate the result (11) for reflexive sheaves explicitly. In particular, we will show that for any torsion-free sheaf $\mathcal{E}$, its dual $\mathcal{E}^{\vee}$ (which is always reflexive) has the property stated. Let $X$ be an affine toric variety with principal cone $\sigma$ and edges $\left\{\tau_{\alpha}\right\}$. Let $A^{\sigma}$ be an equivariant module over $\mathbf{C}\left[\sigma^{\vee}\right]$, and $\left\{A^{\alpha}\right\}$ be the modules associated to the toric divisors. Let $\rho_{\alpha}$ be in the interior of $\tau_{\alpha}^{\perp} \cap \sigma^{\vee}$. Let

$$
\left(A^{\vee}\right)^{\sigma}=\operatorname{Hom}_{\mathbf{C}\left[\sigma^{\vee}\right]}\left(A^{\sigma}, \mathbf{C}\left[\sigma^{\vee}\right]\right)
$$

with associated $T$-action. Now, consider $\left(A^{\vee}\right)^{\sigma}(\chi)$, which is to say equivariant maps $A^{\sigma} \rightarrow \mathbf{C}\left[\sigma^{\vee}\right]^{[-\chi]}$, where the superscript indicates the grading is shifted. For each $\mu \in M, \nu \in \sigma^{\vee}$, we have the commutative diagram

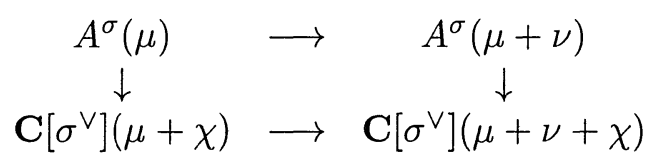

so as $\nu$ gets infinitely deep in $\sigma^{\vee}$, the right edge converges to a map $E \rightarrow \mathbf{C}$, where $E$ is the vector space in which each component of the module $A^{\sigma}$ embeds. More generally for any $\mu$ we have the composition $A^{\sigma}(\mu) \rightarrow E \rightarrow$ $\mathbf{C}$ - thus, an element of $E^{*}=\operatorname{Hom}(E, \mathbf{C})$ completely determines a map $A^{\sigma} \rightarrow \mathbf{C}\left[\sigma^{\vee}\right]^{[-\chi]}$. Note that not any random element of $E^{*}$ can be associated with a map $A^{\sigma} \rightarrow \mathbf{C}\left[\sigma^{\vee}\right]^{[-\chi]}$ : if $\mu \notin \sigma^{\vee}-\chi$, then the image of $A^{\sigma}(\mu)$ must vanish, because the lower left corner of diagram (12) vanishes. Thus, each component $\left(A^{\vee}\right)^{\sigma}(\chi)$ of $\left(A^{\vee}\right)^{\sigma}$ is identified with a subspace of $E^{*}$ that kills certain components of $A^{\sigma}$. Now, this means that

$$
\begin{aligned}
\left(A^{\vee}\right)^{\sigma}(\chi) & =\bigcap_{\mu \notin \sigma^{\vee}}\left(A^{\sigma}(\mu-\chi)\right)^{\perp} \\
& =\bigcap_{\alpha}\left[\bigcap_{\mu \text { s.t. }\left\langle\mu, \tau_{\alpha}\right\rangle<0}\left(A^{\sigma}(\mu-\chi)\right)^{\perp}\right]
\end{aligned}
$$

where the elements on the right hand side should be thought of as subspaces of $E^{*}$. Since $A^{\sigma}(\mu-\chi) \subseteq A^{\alpha}(\mu-\chi)$, we have that $\left(A^{\sigma}(\mu-\chi)\right)^{\perp} \supseteq\left(A^{\alpha}(\mu-\right.$ $\chi))^{\perp}$. More precisely, we have $A^{\sigma}(\mu-\chi) \subseteq A^{\sigma}\left(\mu-\chi+m \rho_{\alpha}\right)$, and for sufficiently large $m$ we have $A^{\sigma}\left(\mu-\chi+m \rho_{\alpha}\right)=A^{\alpha}(\mu-\chi)$, so

$$
\left(A^{\vee}\right)^{\sigma}(\chi)=\bigcap_{\alpha}\left[\bigcap_{\mu \text { s.t. }\left\langle\mu, \tau_{\alpha}\right\rangle<0}\left(A^{\alpha}(\mu-\chi)\right)^{\perp}\right]
$$


Now, we know

$$
\begin{aligned}
\left(A^{\vee}\right)^{\alpha}(\chi) & =\left(A^{\vee}\right)^{\sigma}\left(\chi+m \rho_{\alpha}\right) \text { for large } m \\
& =\bigcap_{\beta}\left[\bigcap_{\mu \text { s.t. }\left\langle\mu, \tau_{\beta}\right\rangle<0}\left(A^{\beta}\left(\mu-\chi-m \rho_{\alpha}\right)\right)^{\perp}\right]
\end{aligned}
$$

Now, for $m$ sufficiently large, we can make $\left\langle\tau_{\beta}, \mu-\chi-m \rho_{\alpha}\right\rangle$ arbitrarily negative if $\alpha \neq \beta$. Thus, for sufficiently large $m$, if $\alpha \neq \beta$ then $A^{\beta}(\mu-\chi-$ $\left.m \rho_{\alpha}\right)=0$, and so $\left(A^{\beta}\left(\mu-\chi-m \rho_{\alpha}\right)\right)^{\perp}=E^{*}$. Thus,

$$
\bigcap_{\mu \text { s.t. }\left\langle\mu, \tau_{\alpha}\right\rangle<0}\left(A^{\alpha}(\mu-\chi)\right)^{\perp}=\left(A^{\vee}\right)^{\alpha}(\chi)
$$

and so plugging into equation (15) we recover

$$
\left(A^{\vee}\right)^{\sigma}(\chi)=\bigcap_{\alpha}\left(A^{\vee}\right)^{\alpha}(\chi)
$$

as was to be shown.

Note that the modules $E^{\alpha}$ associated to toric divisors are all completely specified by filtrations. This is because the affine space associated to each toric divisor is of the form $\mathbf{C} \times\left(\mathbf{C}^{\times}\right)^{k}$ for some $k$, so each $\hat{T}_{\alpha}$ is completely specified by a single index. Thus, even on a singular variety, reflexive sheaves are specified by associating a filtration to each toric divisor.

To review, on both smooth and singular toric varieties, we specify a reflexive sheaf (and also a $G L(r, \mathbf{C})$ bundle) by associating a filtration to each toric divisor. Modules associated with larger cones are then obtained by intersecting the modules associated to toric divisors.

Now, given some reflexive sheaf, how do we determine if it is locally free? On a smooth toric variety, we already know the result - the parabolic subgroups associated to each filtration must satisfy equation (4). On a singular variety, this is necessary but not sufficient. In order for a reflexive sheaf to be locally free, on each element of an affine cover it must look like a direct sum of line bundles. (One line bundle for each generator of the associated freely-generated module.) On a smooth variety, any divisor will define a line bundle, but on a singular toric variety only some divisors (the so-called Cartier divisors) define line bundles. Thus, on a singular toric variety, in addition to checking that equation (4) is satisfied, one must also check that the sheaf splits into a direct sum of line bundles on each maximal cone. The most efficient way to do this is to check that, for any maximal cone, the integers associated to toric divisors à la Kaneyama can be associated with a 
set of Cartier divisors. (Cartier divisors will be studied in greater detail in subsection 4.5.5.)

In order to help clarify these remarks, let us consider an example on $\mathbf{P}^{2}$. Let the fan describing $\mathbf{P}^{2}$ as a toric variety have edges $(1,0),(0,1)$, and $(-1,-1)$, and denote by cone 1 the cone spanned by $(1,0),(0,1)$, cone 2 spanned by $(0,1),(-1,-1)$, and cone 3 spanned by $(-1,-1),(1,0)$. The coordinate charts corresponding to maximal cones are as follows:

$$
\begin{aligned}
U_{1} & =\operatorname{Spec} \mathbf{C}[x, y] \\
U_{2} & =\operatorname{Spec} \mathbf{C}\left[x^{-1}, x^{-1} y\right] \\
U_{3} & =\operatorname{Spec} \mathbf{C}\left[y^{-1}, x y^{-1}\right]
\end{aligned}
$$

Now, to be specific, let us describe an ideal sheaf, call it $\mathcal{I}$, vanishing to order 1 at the origin of cone 1 . The module associated to cone 1 , call it $M_{1}$, is the ideal $(x, y) \subset \mathbf{C}[x, y]$ :

\begin{tabular}{c|cccccc} 
& -2 & -1 & 0 & 1 & 2 & 3 \\
\hline & & & $\vdots$ & & & \\
3 & & 0 & $\mathbf{C}$ & $\mathbf{C}$ & $\mathbf{C}$ & \\
2 & & 0 & $\mathbf{C}$ & $\mathbf{C}$ & $\mathbf{C}$ & \\
1 & $\cdots$ & 0 & $\mathbf{C}$ & $\mathbf{C}$ & $\mathbf{C}$ & $\cdots$ \\
0 & & 0 & 0 & $\mathbf{C}$ & $\mathbf{C}$ & \\
-1 & & 0 & 0 & 0 & 0 & \\
& & & $\vdots$ & & &
\end{tabular}

Denote the module over $U_{2}$ by $M_{2}$. As the ideal sheaf is trivial in cone 2, $M_{2}$ should correspond to the structure sheaf on $U_{2}$ (meaning, $M_{2}=$ $\left.\mathbf{C}\left[x^{-1}, x^{-1} y\right]\right)$ :

\begin{tabular}{c|ccccccc} 
& -4 & -3 & -2 & -1 & 0 & 1 & 2 \\
\hline & & & & $\vdots$ & & & \\
3 & & $\mathbf{C}$ & 0 & 0 & 0 & 0 & \\
2 & & $\mathbf{C}$ & $\mathbf{C}$ & 0 & 0 & 0 & \\
1 & $\cdots$ & $\mathbf{C}$ & $\mathbf{C}$ & $\mathbf{C}$ & 0 & 0 & $\cdots$ \\
0 & & $\mathbf{C}$ & $\mathbf{C}$ & $\mathbf{C}$ & $\mathbf{C}$ & 0 & \\
-1 & & 0 & 0 & 0 & 0 & 0 & \\
& & & & $\vdots$ & & &
\end{tabular}

Denote the module over $U_{3}$ by $M_{3}$. As for cone 2, the module $M_{3}$ should also correspond to the structure sheaf on $U_{3}$ (meaning, $M_{3}=\mathbf{C}\left[y^{-1}, x y^{-1}\right]$ ): 


\begin{tabular}{c|cccccc} 
& -2 & -1 & 0 & 1 & 2 & 3 \\
\hline & & & $\vdots$ & & & \\
1 & & 0 & 0 & 0 & 0 & \\
0 & $\cdots$ & 0 & $\mathbf{C}$ & 0 & 0 & $\cdots$ \\
-1 & & 0 & $\mathbf{C}$ & $\mathbf{C}$ & 0 & \\
-2 & & 0 & $\mathbf{C}$ & $\mathbf{C}$ & $\mathbf{C}$ & \\
& & & $\vdots$ & & &
\end{tabular}

Now, the edge at the intersection of cones 1,2 corresponds to the affine variety

$$
U_{12}=\operatorname{Spec} \mathbf{C}\left[x, x^{-1}, y\right]
$$

and similarly for the other edges

$$
\begin{aligned}
& U_{13}=\operatorname{Spec} \mathbf{C}\left[y, y^{-1}, x\right] \\
& U_{23}=\operatorname{Spec} \mathbf{C}\left[x^{-1} y, x y^{-1}, x^{-1}\right]
\end{aligned}
$$

The module $M_{12}$ corresponding to $U_{12}$ is simply

\begin{tabular}{c|ccccc} 
& -2 & -1 & 0 & 1 & 2 \\
\hline & & & $\vdots$ & & \\
2 & & $\mathbf{C}$ & $\mathbf{C}$ & $\mathbf{C}$ & \\
1 & & $\mathbf{C}$ & $\mathbf{C}$ & $\mathbf{C}$ & \\
0 & $\cdots$ & $\mathbf{C}$ & $\mathbf{C}$ & $\mathbf{C}$ & $\cdots$ \\
-1 & & 0 & 0 & 0 & \\
& & & $\vdots$ & &
\end{tabular}

where we have written it out over $M$ rather than $\hat{T}_{12}$. (It should be clear that the module is constant along directions in $v_{12}^{\perp}$, where $v_{12}$ is the edge of the fan bordering cones 1,2 .)

The module $M_{13}$ corresponding to $U_{13}$ is simply

\begin{tabular}{r|ccccc} 
& -2 & -1 & 0 & 1 & 2 \\
\hline & & & $\vdots$ & & \\
1 & & 0 & $\mathbf{C}$ & $\mathbf{C}$ & \\
0 & $\cdots$ & 0 & $\mathbf{C}$ & $\mathbf{C}$ & $\cdots$ \\
-1 & & 0 & $\mathbf{C}$ & $\mathbf{C}$ & \\
& & & $\vdots$ & &
\end{tabular}


The module $M_{23}$ corresponding to $U_{23}$ is simply

\begin{tabular}{c|ccccccccc} 
& -4 & -3 & -2 & -1 & 0 & 1 & 2 & 3 & 4 \\
\hline & & & & & $\vdots$ & & & & \\
1 & & $\mathbf{C}$ & $\mathbf{C}$ & $\mathbf{C}$ & 0 & 0 & 0 & 0 & \\
0 & $\cdots$ & $\mathbf{C}$ & $\mathbf{C}$ & $\mathbf{C}$ & $\mathbf{C}$ & 0 & 0 & 0 & $\cdots$ \\
-1 & & $\mathbf{C}$ & $\mathbf{C}$ & $\mathbf{C}$ & $\mathbf{C}$ & $\mathbf{C}$ & 0 & 0 & \\
-2 & & $\mathbf{C}$ & $\mathbf{C}$ & $\mathbf{C}$ & $\mathbf{C}$ & $\mathbf{C}$ & $\mathbf{C}$ & 0 & \\
& & & & & $\vdots$ & & & &
\end{tabular}

It is easy to check that the inclusion relations advertised are satisfied in each case. It is also easy to check that $\mathcal{I}^{\vee \vee} \cong \mathcal{O}$, the structure sheaf, as expected.

In the case of a smooth compact toric variety, as above, we can perform an $S L(2, \mathbf{Z})$ transformation on each maximal cone to rotate them to all be of the same form. Given cones in such a standard form, we can then work somewhat more elegantly. Such an approach is described in the next subsection.

In later subsections we will speak to singular toric varieties at greater length.

\subsubsection{Torsion-free Sheaves on Smooth Toric Varieties}

In this section we will specialize to torsion-free sheaves on smooth toric varieties, as has been discussed in [4].

According to [4], to describe a torsion-free sheaf, we generalize the filtrations discussed earlier, to multifiltrations

$$
E^{\sigma}(I)=E^{\left(\alpha_{1}, \alpha_{2}, \ldots, \alpha_{k}\right)}\left(i_{1}, i_{2}, \ldots, i_{k}\right)
$$

for $\sigma=\left(\alpha_{1}, \alpha_{2}, \ldots, \alpha_{k}\right) \in \Sigma$ and $I=\left(i_{1}, i_{2}, \ldots, i_{k}\right), i \in \mathbf{Z}$. These filtrations are nonincreasing: for all $k$,

$$
E^{\sigma}\left(i_{1}, \ldots, i_{k}, \ldots, i_{p}\right) \supseteq E^{\sigma}\left(i_{1}, \ldots, i_{k}+1, \ldots, i_{p}\right)
$$

These multifiltrations must obey the compatibility condition that for every pair of cones $\tau \subseteq \sigma, \tau=\left(\alpha_{1}, \alpha_{2}, \ldots, \alpha_{p}\right), \sigma=\left(\alpha_{1}, \alpha_{2}, \ldots, \alpha_{p}, \beta_{1}, \ldots, \beta_{q}\right)$,

$$
E^{\tau}\left(i_{1}, i_{2}, \ldots, i_{p}\right)=E^{\sigma}\left(i_{1}, i_{2}, \ldots, i_{p},-\infty, \ldots,-\infty\right)
$$


This compatibility condition is not related to the compatibility condition for bundles, but rather is the compatibility condition on inclusion morphisms usually used to define sheaves.

Chern class computations, for example, can be carried out using simple modifications of the formulae given earlier. Instead of calculating $E^{\sigma}\left(i_{1}, \ldots i_{k}\right)$ using formula (6), use the multifiltration defining the torsion-free sheaf. Formula (7) must be generalized to

$$
E^{[\sigma]}\left(i_{1}, i_{2}, \ldots, i_{p}\right)=\triangle_{1} \triangle_{2} \cdots \triangle_{p} E^{\sigma}\left(i_{1}, i_{2}, \ldots, i_{p}\right)
$$

where $\triangle_{k}$ is a difference operator yielding a formal difference of vector spaces $\triangle_{k} E^{\sigma}\left(i_{1}, \ldots, i_{k}, \ldots, i_{p}\right)=E^{\sigma}\left(i_{1}, \ldots, i_{k}, \ldots, i_{p}\right)-E^{\sigma}\left(i_{1}, \ldots, i_{k}+1, \ldots, i_{p}\right)$

For example,

$\operatorname{dim} \triangle_{k} E^{\sigma}\left(i_{1}, \ldots, i_{k}, \ldots, i_{p}\right)=\operatorname{dim} E^{\sigma}\left(i_{1}, \ldots, i_{k}, \ldots, i_{p}\right)-\operatorname{dim} E^{\sigma}\left(i_{1}, \ldots, i_{k}+1, \ldots, i_{p}\right)$

In particular, the dimension of $E^{[\sigma]}(I)$ may be negative. Given these redefinitions, one may then use equations (9), (8) to calculate the Chern character and total Chern class, respectively.

Sheaf cohomology can be calculated precisely as before, with the note that each $E^{\sigma}$ should be reinterpreted as the multifiltration associated to cone $\sigma$, rather than the intersection of the filtrations on the toric divisors bounding $\sigma$.

How are reflexive sheaves described in this language? According to [4], given a torsion-free sheaf $\mathcal{E}$ described by a family of compatible multifiltrations $E^{\sigma}$, its bidual sheaf $\mathcal{E}^{\vee \vee}$ is given by the family of multifiltrations

$$
E^{\vee \vee\left(\alpha_{1}, \alpha_{2}, \ldots, \alpha_{p}\right)}\left(i_{1}, i_{2}, \ldots, i_{p}\right)=E^{\alpha_{1}}\left(i_{1}\right) \cap \cdots \cap E^{\alpha_{p}}\left(i_{p}\right)
$$

In particular, if $\mathcal{E}$ is reflexive $\left(\mathcal{E}=\mathcal{E}^{\vee \vee}\right)$, then the multifiltration is completely specified by a set of ordinary filtrations.

How does a reflexive sheaf differ from a locally free sheaf (i.e., a vector bundle)? The filtrations defining a vector bundle must satisfy a compatibility condition (not related to the compatibility condition for torsion-free sheaves), whereas the filtrations defining a reflexive sheaf are not required to satisfy any compatibility conditions - any random set of filtrations defines a reflexive sheaf.

In particular, note that a locally free sheaf is a special case of a reflexive sheaf. 
Also note that if we view a reflexive sheaf as a locally free sheaf with singularities, then the singularities are located at the intersections of toric divisors not satisfying the bundle compatibility conditions. As filtrations along any two toric divisors automatically satisfy the bundle compatibility conditions, we implicitly verify that singularities of a reflexive sheaf are located at codimension 3 .

As a check, note that we have verified the standard fact that reflexive sheaves on a smooth complex surface are locally free, at least for equivariant sheaves on toric surfaces. In this language, this follows from the fact that if the maximal cones in the fan are two-dimensional, then the compatibility condition on the filtrations is obeyed trivially - any pair of filtrations is automatically compatible in the sense needed to define a vector bundle, and so on a toric surface reflexive sheaves are defined by precisely the same data as vector bundles.

In general, given a torsion free sheaf $\mathcal{E}$, there is a natural map $\mathcal{E} \rightarrow \mathcal{E}^{\vee \vee}$. How can this map be seen in the present language? First, note that the sheaf compatibility conditions in equation (16) mean $\tau \subseteq \sigma$ implies

$$
E^{\sigma}\left(i_{1}, \ldots, i_{p}, j_{1}, \ldots, j_{k}\right) \subseteq E^{\tau}\left(i_{1}, \ldots, i_{p}\right)
$$

and in particular, for all $k$

$$
E^{\left(\alpha_{1}, \cdots, \alpha_{p}\right)}\left(i_{1}, \ldots, i_{p}\right) \subseteq E^{\alpha_{k}}\left(i_{k}\right)
$$

so clearly

$$
E^{\left(\alpha_{1}, \cdots, \alpha_{p}\right)}\left(i_{1}, \ldots, i_{p}\right) \subseteq E^{\alpha_{1}}\left(i_{1}\right) \cap \cdots \cap E^{\alpha_{p}}\left(i_{p}\right)=E^{\vee \vee\left(\alpha_{1}, \cdots, \alpha_{p}\right)}\left(i_{1}, \ldots, i_{p}\right)
$$

This technology now allows us to gain a better grasp of the sheaves appearing in extremal transitions (previous studies of this matter have appeared in [13]). Given a set of compatible filtrations defining a vector bundle, by blowing down one of the toric divisors we are clearly left with a set of filtrations, which no longer necessarily satisfy the usual (bundle) compatibility conditions - so we get a reflexive sheaf. In other words, when blowing down a toric divisor, there exists a natural transformation of (equivariant) bundles into (equivariant) reflexive sheaves.

Note that the sheaves obtained in this manner are not the same sheaves one obtains by pushforward along the blowdown morphism. For example, consider an equivariant line bundle on the Hirzebruch surface $\mathbf{F}_{1}$, the blowup of $\mathbf{P}^{2}$ at a point. In Klyachko's formalism, if we blowdown $\mathbf{F}_{1} \rightarrow \mathbf{P}^{2}$, we recover another equivariant line bundle (as reflexive sheaves on a surface 
are locally free). By contrast, if we pushforward the line bundle along the blowdown morphism, we will get a torsion-free sheaf, which in general will not be locally free.

\subsubsection{Global Ext}

In principle it is possible to calculate global Ext groups for equivariant torsion-free sheaves on smooth toric varieties, with a calculation analogous to the sheaf cohomology group calculation in section 4.3. For completeness it is mentioned here.

The group (global) $\operatorname{Ext}^{i}(\mathcal{E}, \mathcal{F})$ is given by the limit of the spectral sequence whose first-level terms are

$$
E_{1}^{p, q}=\bigoplus_{\text {codim } \sigma=p} \operatorname{Ext}^{q}\left(\left.\mathcal{E}\right|_{U_{\sigma}},\left.\mathcal{F}\right|_{U_{\sigma}}\right)
$$

where $\sigma$ denotes a cone, and $\left.\mathcal{E}\right|_{U_{\sigma}}$ denotes the module of sections of $\mathcal{E}$ over the open set $U_{\sigma}$. As noted earlier, when $\mathcal{E}$ is equivariant and torsion-free, this module of sections is precisely the graded module $E^{\sigma}$ defining the equivariant torsion-free sheaf $\mathcal{E}$.

Like the sheaf cohomology groups, the groups $\operatorname{Ext}^{i}(\mathcal{E}, \mathcal{F})$ have an isotypic decomposition when $\mathcal{E}, \mathcal{F}$ are both equivariant:

$$
\text { (global) } \operatorname{Ext}^{i}(\mathcal{E}, \mathcal{F})=\oplus_{\chi} \operatorname{Ext}^{i}(\mathcal{E}, \mathcal{F})_{\chi}
$$

If we let $\left\{E^{\sigma}\right\},\left\{F^{\sigma}\right\}$ denote the corresponding sets of data, then the group $\operatorname{Ext}^{i}(\mathcal{E}, \mathcal{F})_{\chi}$ is given by the limit of the spectral sequence with first-level terms

$$
E_{1}^{p, q}=\bigoplus_{\text {codim } \sigma=p} \operatorname{Ext}^{q}\left(E^{\sigma}, F^{\sigma}\right)_{\chi}
$$

Unfortunately, it is not clear at present how this can be made more computationally effective.

As a check, note that when $\mathcal{E}$ is locally free, we recover the sheaf cohomology of $\mathcal{E}^{\vee} \otimes \mathcal{F}$. When $\mathcal{E}$ is locally free, $E^{\sigma}$ is a freely generated module for all $\sigma$, and so

$$
\operatorname{Ext}^{q}\left(E^{\sigma}, F^{\sigma}\right)=0
$$

for all $q>0$. Recall that the differential

$$
d_{r}: E_{r}^{p, q} \rightarrow E_{r}^{p+r, q-r+1}
$$




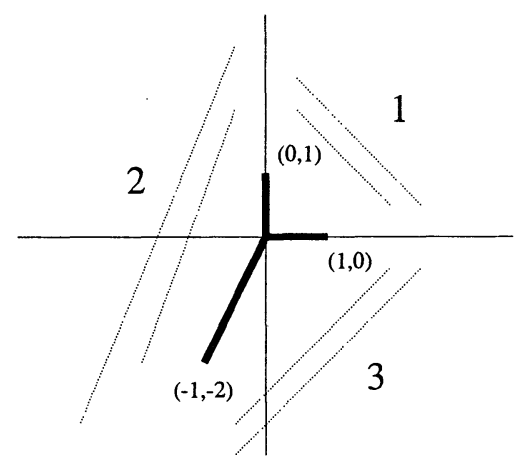

Figure 1: A fan describing the weighted projective space $\mathbf{P}_{1,1,2}^{2}$ as a toric variety.

so in particular

$$
d_{1}: E_{1}^{p, q} \rightarrow E_{1}^{p+1, q}
$$

and our spectral sequence degenerates to the complex defining sheaf cohomology in section 4.3.2.

\subsubsection{An Example on $\mathbf{P}_{1,1,2}^{2}$}

First, we shall make some general remarks on the equivariant structure of modules describing torsion-free sheaves on $\mathbf{P}_{1,1,2}^{2}$. The fan for $\mathbf{P}_{1,1,2}^{2}$ can be taken to have edges $(1,0),(0,1)$, and $(-1,-2)$, as shown in figure 1 . Denote as cone 1 the cone spanned by $(1,0)$ and $(0,1)$. Denote as cone 2 the cone spanned by $(0,1)$ and $(-1,-2)$. Denote as cone 3 the cone spanned by $(-1,-2)$ and $(1,0)$.

To be specific, we shall describe an ideal sheaf on $\mathbf{P}_{1,1,2}^{2}$ which vanishes to order 1 at the origin of cone 1.

The affine space $U_{1}$ corresponding to cone 1 is Spec $\mathbf{C}[x, y]$, and a torsionfree module $E^{\sigma_{1}}$ is a bifiltration with inclusions

$$
\begin{aligned}
E^{\sigma_{1}}\left(i_{1}, i_{2}\right) & \hookrightarrow E^{\sigma_{1}}\left(i_{1}+1, i_{2}\right) \\
& \hookrightarrow E^{\sigma_{1}}\left(i_{1}, i_{2}+1\right)
\end{aligned}
$$

The module $M_{1}$ on $U_{1}$ for our example is the ideal $(x, y) \subset \mathbf{C}[x, y]$ : 


\begin{tabular}{c|cccccc} 
& -2 & -1 & 0 & 1 & 2 & 3 \\
\hline & & & $\vdots$ & & & \\
2 & & 0 & $\mathbf{C}$ & $\mathbf{C}$ & $\mathbf{C}$ & \\
1 & & 0 & $\mathbf{C}$ & $\mathbf{C}$ & $\mathbf{C}$ & \\
0 & $\cdots$ & 0 & 0 & $\mathbf{C}$ & $\mathbf{C}$ & $\cdots$ \\
-1 & & 0 & 0 & 0 & 0 & \\
& & & $\vdots$ & & &
\end{tabular}

The affine space $U_{2}$ corresponding to cone 2 is $\operatorname{Spec} \mathbf{C}\left[x^{-1}, x^{-2} y\right]$, and a torsion-free module $E^{\sigma_{2}}$ is a bifiltration with inclusions

$$
\begin{aligned}
E^{\sigma_{2}}\left(i_{1}, i_{2}\right) & \hookrightarrow E^{\sigma_{2}}\left(i_{1}-1, i_{2}\right) \\
& \hookrightarrow E^{\sigma_{2}}\left(i_{1}-2, i_{2}+1\right)
\end{aligned}
$$

The module $M_{2}$ on $U_{2}$ for our example should describe the structure sheaf on $U_{2}$, and in fact $M_{2}=\mathbf{C}\left[x^{-1}, x^{-2} y\right]$ :

\begin{tabular}{c|cccccccc} 
& -5 & -4 & -3 & -2 & -1 & 0 & 1 & 2 \\
\hline & & & & $\vdots$ & & & & \\
2 & & $\mathbf{C}$ & 0 & 0 & 0 & 0 & 0 & \\
1 & & $\mathbf{C}$ & $\mathbf{C}$ & $\mathbf{C}$ & 0 & 0 & 0 & \\
0 & $\cdots$ & $\mathbf{C}$ & $\mathbf{C}$ & $\mathbf{C}$ & $\mathbf{C}$ & $\mathbf{C}$ & 0 & $\cdots$ \\
-1 & & 0 & 0 & 0 & 0 & 0 & 0 & \\
& & & & $\vdots$ & & & &
\end{tabular}

The affine space $U_{3}$ corresponding to cone 3 is Spec $\mathbf{C}\left[y^{-1}, x y^{-1}, x^{2} y^{-1}\right]$, which contains a $\mathbf{C}^{2} / \mathbf{Z}_{2}$ singularity. A torsion-free module $E^{\sigma_{3}}$ is a bifiltration with inclusions

$$
\begin{aligned}
E^{\sigma_{3}}\left(i_{1}, i_{2}\right) & \hookrightarrow E^{\sigma_{3}}\left(i_{1}, i_{2}-1\right) \\
& \hookrightarrow E^{\sigma_{3}}\left(i_{1}+1, i_{2}-1\right) \\
& \hookrightarrow E^{\sigma_{3}}\left(i_{1}+2, i_{2}-1\right)
\end{aligned}
$$

The module $M_{3}$ on $U_{3}$ for our example should describe the structure sheaf on $U_{3}$, and in fact $M_{3}=\mathbf{C}\left[y^{-1}, x y^{-1}, x^{2} y^{-1}\right]$ : 


\begin{tabular}{c|cccccccccc} 
& -2 & -1 & 0 & 1 & 2 & 3 & 4 & 5 & 6 & 7 \\
\hline & & & & & $\vdots$ & & & & & \\
1 & & 0 & 0 & 0 & 0 & 0 & 0 & 0 & 0 & \\
0 & & 0 & $\mathbf{C}$ & 0 & 0 & 0 & 0 & 0 & 0 & \\
-1 & $\cdots$ & 0 & $\mathbf{C}$ & $\mathbf{C}$ & $\mathbf{C}$ & 0 & 0 & 0 & 0 & $\cdots$ \\
-2 & & 0 & $\mathbf{C}$ & $\mathbf{C}$ & $\mathbf{C}$ & $\mathbf{C}$ & $\mathbf{C}$ & 0 & 0 & \\
-3 & & 0 & $\mathbf{C}$ & $\mathbf{C}$ & $\mathbf{C}$ & $\mathbf{C}$ & $\mathbf{C}$ & $\mathbf{C}$ & $\mathbf{C}$ & \\
& & & & & $\vdots$ & & & & &
\end{tabular}

How are these three bifiltrations glued together? We shall examine modules over the intersections of the cones.

The affine space $U_{12}$ at the intersection of cones 1 and 2 is Spec $\mathbf{C}\left[x, x^{-1}, y\right]$, and a torsion-free module $E^{\sigma_{12}}$ over this space is a bifiltration with inclusions

$$
\begin{aligned}
E^{\sigma_{12}}\left(i_{1}, i_{2}\right) & \hookrightarrow E^{\sigma_{12}}\left(i_{1}+1, i_{2}\right) \\
& \hookrightarrow E^{\sigma_{12}}\left(i_{1}-1, i_{2}\right) \\
& \hookrightarrow E^{\sigma_{12}}\left(i_{1}, i_{2}+1\right)
\end{aligned}
$$

For our example, the module $M_{12}$ associated to $U_{12}$ is the ring $\mathbf{C}\left[x, x^{-1}, y\right]$ :

\begin{tabular}{c|ccccc} 
& -2 & -1 & 0 & 1 & 2 \\
\hline & & & $\vdots$ & & \\
2 & & $\mathbf{C}$ & $\mathbf{C}$ & $\mathbf{C}$ & \\
1 & $\cdots$ & $\mathbf{C}$ & $\mathbf{C}$ & $\mathbf{C}$ & $\cdots$ \\
0 & & $\mathbf{C}$ & $\mathbf{C}$ & $\mathbf{C}$ & \\
-1 & & 0 & 0 & 0 & \\
& & & $\vdots$ & &
\end{tabular}

The affine space $U_{13}$ at the intersection of cones 1 and 3 is $\operatorname{Spec} \mathbf{C}\left[x, y, y^{-1}\right]$, and a torsion-free module $E^{\sigma_{13}}$ over this space is a bifiltration with inclusions

$$
\begin{aligned}
E^{\sigma_{13}}\left(i_{1}, i_{2}\right) & \hookrightarrow E^{\sigma_{13}}\left(i_{1}+1, i_{2}\right) \\
& \hookrightarrow E^{\sigma_{13}}\left(i_{1}, i_{2}+1\right) \\
& \hookrightarrow E^{\sigma_{13}}\left(i_{1}, i_{2}-1\right)
\end{aligned}
$$

For our example, the module $M_{13}$ associated to $U_{13}$ is the ring $\mathbf{C}\left[x, y, y^{-1}\right]$ : 


\begin{tabular}{c|cccccc} 
& -2 & -1 & 0 & 1 & 2 & 3 \\
\hline & & & $\vdots$ & & & \\
1 & & 0 & $\mathbf{C}$ & $\mathbf{C}$ & $\mathbf{C}$ & \\
0 & $\cdots$ & 0 & $\mathbf{C}$ & $\mathbf{C}$ & $\mathbf{C}$ & $\cdots$ \\
-1 & & 0 & $\mathbf{C}$ & $\mathbf{C}$ & $\mathbf{C}$ & \\
& & & $\vdots$ & & &
\end{tabular}

The affine space $U_{23}$ at the intersection of cones 2 and 3 is Spec $\mathbf{C}\left[x^{-2} y, x^{2} y^{-1}, x y^{-1}\right]$, and a torsion-free module $E^{\sigma_{23}}$ over this space is a bifiltration with inclusions

$$
\begin{aligned}
E^{\sigma_{23}}\left(i_{1}, i_{2}\right) & \hookrightarrow E^{\sigma_{23}}\left(i_{1}-2, i_{2}+1\right) \\
& \hookrightarrow E^{\sigma_{23}}\left(i_{1}+2, i_{2}-1\right) \\
& \hookrightarrow E^{\sigma_{23}}\left(i_{1}+1, i_{2}-1\right)
\end{aligned}
$$

For our example, the module $M_{23}$ associated to $U_{23}$ is the ring $\mathbf{C}\left[x^{-2} y, x^{2} y^{-1}, x y^{-1}\right]$ :

\begin{tabular}{c|ccccccccc} 
& -5 & -4 & -3 & -2 & -1 & 0 & 1 & 2 & 3 \\
\hline & & & & & $\vdots$ & & & & \\
2 & & $\mathbf{C}$ & 0 & 0 & 0 & 0 & 0 & 0 & \\
1 & & $\mathbf{C}$ & $\mathbf{C}$ & $\mathbf{C}$ & 0 & 0 & 0 & 0 & \\
0 & $\cdots$ & $\mathbf{C}$ & $\mathbf{C}$ & $\mathbf{C}$ & $\mathbf{C}$ & $\mathbf{C}$ & 0 & 0 & $\cdots$ \\
-1 & & $\mathbf{C}$ & $\mathbf{C}$ & $\mathbf{C}$ & $\mathbf{C}$ & $\mathbf{C}$ & $\mathbf{C}$ & $\mathbf{C}$ & \\
& & & & & $\vdots$ & & & &
\end{tabular}

In passing we should note that toric varieties can have singularities worse than orbifold singularities - for example, an affine conifold singularity in three dimensions is toric. For completeness, we very briefly review the conifold singularity below.

The affine conifold singularity in three dimensions is the hypersurface $a d-b c=0$ in $\mathbf{C}[a, b, c, d]$. It can be described as a (noncompact) toric variety. The fan has edges $(-1,0,1),(0,-1,1),(-1,1,0)$, and $(1,-1,0)$, describing a single cone in three dimensions (in the region $z \geq 0, x+z \geq 0$, $y+z \geq 0$, and $x+y+z \geq 0)$. Its dual cone has the edges $(0,0,1),(1,0,1)$, $(0,1,1)$, and $(1,1,1)$.

Any torsion-free sheaf on this affine space will be described in bulk by a $\mathbf{Z}^{3}$-graded module $E$, with inclusions

$$
\begin{aligned}
E\left(i_{1}, i_{2}, i_{3}\right) & \hookrightarrow E\left(i_{1}, i_{2}, i_{3}+1\right) \\
& \hookrightarrow E\left(i_{1}+1, i_{2}, i_{3}+1\right)
\end{aligned}
$$




$$
\begin{aligned}
& \hookrightarrow E\left(i_{1}, i_{2}+1, i_{3}+1\right) \\
& \hookrightarrow E\left(i_{1}+1, i_{2}+1, i_{3}+1\right)
\end{aligned}
$$

\subsubsection{Cartier Divisors vs Weil Divisors}

On a smooth variety, for any divisor there exists a corresponding line bundle, a standard fact well-known to physicists. On singular varieties, not all divisors correspond to line bundles. In general, an arbitrary divisor is called a Weil divisor, and a Weil divisor that happens to correspond to a line bundle (rather than some other rank 1 sheaf) is called a Cartier divisor ${ }^{20}$. Essentially, a divisor will not necessarily define a line bundle when it intersects a singularity.

Put another way, on any normal variety (of which toric varieties are examples) an arbitrary (Weil) divisor will yield a reflexive rank 1 sheaf [57, 58]. In the special case that the reflexive rank 1 sheaf is a line bundle, we say the Weil divisor is Cartier. On a smooth variety, all reflexive rank 1 sheaves are line bundles, thus on a smooth variety all Weil divisors are Cartier.

In brief, for a $T$-invariant divisor $D$ on an affine toric space $U_{\sigma}$ to be Cartier, there must exist a character ${ }^{21} \chi$ such that

$$
D=\sum_{\alpha}\left\langle v_{\alpha}, \chi\right\rangle D_{\alpha}
$$

where $D_{\alpha}$ is the divisor associated to the edge of the fan $v_{\alpha}$.

Let us work out a specific example in detail. Consider the affine space $U=\mathbf{C}^{2} / \mathbf{Z}_{2}$ described as a toric variety - the corresponding fan has edges

$$
\begin{aligned}
& v_{1}=(2,-1) \\
& v_{2}=(0,1)
\end{aligned}
$$

The affine space $U=\operatorname{Spec} \mathbf{C}\left[x, x y, x y^{2}\right]=\operatorname{Spec} \mathbf{C}[x, y, z] /\left(x y=z^{2}\right)$. The affine space corresponding to divisor 1 is $U_{1}=\operatorname{Spec} \mathbf{C}\left[x y^{2}, x^{-1} y^{-2}, y^{-1}\right]$, and the affine space corresponding to divisor 2 is $U_{2}=\operatorname{Spec} \mathbf{C}\left[x, x^{-1}, y\right]$.

Now, let us consider the ideal sheaf defined by the principal ideal $(x)$. Since this ideal sheaf is defined by a principal ideal, it should correspond to a Cartier divisor. Indeed, the corresponding module on $U$ is

\footnotetext{
${ }^{20}$ Technical fiends will note we are deliberately being sloppy, for reasons of readability.

${ }^{21}$ Technical fiends will recognize that the character $\chi$ is the location of the generator of the principal fractional ideal.
} 


\begin{tabular}{c|cccccc} 
& -2 & -1 & 0 & 1 & 2 & 3 \\
\hline & & & $\vdots$ & & & \\
4 & & 0 & 0 & 0 & $\mathbf{C}$ & \\
3 & & 0 & 0 & 0 & $\mathbf{C}$ & \\
2 & & 0 & 0 & $\mathbf{C}$ & $\mathbf{C}$ & \\
1 & $\cdots$ & 0 & 0 & $\mathbf{C}$ & $\mathbf{C}$ & $\cdots$ \\
0 & & 0 & $\mathbf{C}$ & $\mathbf{C}$ & $\mathbf{C}$ & \\
-1 & & 0 & 0 & 0 & 0 & \\
& & & $\vdots$ & & &
\end{tabular}

It is manifestly obvious that this module is freely generated (and therefore a line bundle), with generator located at $i_{1}=i_{2}=0$.

As this ideal sheaf is a line bundle, it should be defined by some divisor on the toric variety. Which divisor? To determine the corresponding divisor, we first find the modules $M_{1}, M_{2}$ over the affine spaces $U_{1}, U_{2}$. The module $M_{1}$ is given by

\begin{tabular}{c|ccccccc} 
& -2 & -1 & 0 & 1 & 2 & 3 & 4 \\
\hline & & & & $\vdots$ & & & \\
4 & & 0 & 0 & 0 & 0 & $\mathbf{C}$ & \\
3 & & 0 & 0 & 0 & 0 & $\mathbf{C}$ & \\
2 & & 0 & 0 & 0 & $\mathbf{C}$ & $\mathbf{C}$ & \\
1 & & 0 & 0 & 0 & $\mathbf{C}$ & $\mathbf{C}$ & \\
0 & $\cdots$ & 0 & 0 & $\mathbf{C}$ & $\mathbf{C}$ & $\mathbf{C}$ & $\ldots$ \\
-1 & & 0 & 0 & $\mathbf{C}$ & $\mathbf{C}$ & $\mathbf{C}$ & \\
-2 & & 0 & $\mathbf{C}$ & $\mathbf{C}$ & $\mathbf{C}$ & $\mathbf{C}$ & \\
-3 & & 0 & $\mathbf{C}$ & $\mathbf{C}$ & $\mathbf{C}$ & $\mathbf{C}$ & \\
-4 & & $\mathbf{C}$ & $\mathbf{C}$ & $\mathbf{C}$ & $\mathbf{C}$ & $\mathbf{C}$ & \\
& & & & $\vdots$ & & &
\end{tabular}

and the module $M_{2}$ is given by

\begin{tabular}{c|ccccc} 
& -2 & -1 & 0 & 1 & 2 \\
\hline & & & $\vdots$ & & \\
2 & & $\mathbf{C}$ & $\mathbf{C}$ & $\mathbf{C}$ & \\
1 & & $\mathbf{C}$ & $\mathbf{C}$ & $\mathbf{C}$ & \\
0 & $\cdots$ & $\mathbf{C}$ & $\mathbf{C}$ & $\mathbf{C}$ & $\cdots$ \\
-1 & & 0 & 0 & 0 & \\
& & & $\vdots$ & &
\end{tabular}


Now, in each case, to determine the component of the divisor along $D_{i}$ we compute $\left\langle v_{i}, \chi_{i}\right\rangle$ where $\chi_{i}$ is the location of the generator of $M_{i}$. (Each generator is only defined up to an element of $v_{i}^{\perp}$, but of course that will not matter for getting a number.) It is easy to show that

$$
\begin{aligned}
& \left\langle v_{1}, \chi_{1}\right\rangle=2 \\
& \left\langle v_{2}, \chi_{2}\right\rangle=0
\end{aligned}
$$

so the ideal sheaf defined by the ideal $(x)$ is precisely the line bundle with divisor $-2 D_{1}$. (Note we can choose $\chi_{1}=\chi_{2}$, in accordance with the fact this divisor is Cartier.) Indeed, it is an exercise in [8] that $-2 D_{1}$ is a Cartier divisor in this example.

Now, in this example, the divisor $-D_{1}$ is not Cartier, but merely Weil. What sheaf does this correspond to? We shall simply state the result that the corresponding module on $U$ is

\begin{tabular}{c|cccccc} 
& -1 & 0 & 1 & 2 & 3 & 4 \\
\hline & & & $\vdots$ & & & \\
4 & & 0 & 0 & 0 & $\mathbf{C}$ & \\
3 & & 0 & 0 & $\mathbf{C}$ & $\mathbf{C}$ & \\
2 & & 0 & 0 & $\mathbf{C}$ & $\mathbf{C}$ & \\
1 & $\cdots$ & 0 & $\mathbf{C}$ & $\mathbf{C}$ & $\mathbf{C}$ & $\cdots$ \\
0 & & 0 & $\mathbf{C}$ & $\mathbf{C}$ & $\mathbf{C}$ & \\
-1 & & 0 & 0 & 0 & 0 & \\
& & & $\vdots$ & & &
\end{tabular}

This module is precisely the ideal $(x, x y)$. It should be obvious that this module is not freely generated, but rather is defined by two generators (at $(1,0)$ and $(1,1))$ and one relation. (Since the module is not freely generated, the rank 1 sheaf does not correspond to a line bundle.) Although this module is not freely generated, it is easy to check that the modules $M_{i}$ associated to either toric divisor are freely generated. Also, note that this module is reflexive. On a smooth variety, a reflexive rank 1 sheaf is precisely a line bundle - here we see explicitly that on a singular variety we can have reflexive rank 1 sheaves which are not bundles.

\section{Moduli Spaces of Equivariant Sheaves}

To construct a moduli space of, say, equivariant reflexive sheaves, we should take the space of all filtrations of fixed Chern classes and quotient by $G L(r, \mathbf{C})$ 
equivalences. In doing so, we would find that some of the sheaves should be omitted before quotienting in order to get a well-behaved (for example, Hausdorff) result. This modified quotient is known formally as a GIT (Geometric Invariant Theory) quotient, and the sheaves omitted before quotienting are labelled "unstable."

In many ways GIT quotients ${ }^{22}$ are the prototypes for moduli spaces, in that many features of moduli space problems have analogues within GIT quotients. In this section we shall see that in the special case of moduli spaces of equivariant sheaves, the moduli spaces are precisely GIT quotients.

In section 5.1 we begin by describing Mumford-Takemoto stability - in other words, we describe the sheaves we wish to include in any given moduli space. In section 5.2 we work out the moduli space of equivariant reflexive sheaves, as a GIT quotient of a closed subset of a product of flag manifolds, in considerable detail. In particular we show explicitly that the GIT quotient realizes Mumford-Takemoto stability. In section 5.3 we describe moduli spaces of equivariant bundles, and study orbifold singularities occurring in the moduli space of $G$-bundles. In section 5.4 we briefly speak to moduli spaces of equivariant torsion-free sheaves, and in section 5.5 we remark on the interrelation of the moduli spaces of equivariant reflexive and torsion-free sheaves.

Much of section 5.2.1 is a generalization of results of A. A. Klyachko [3].

\section{$5.1 \quad$ Stability}

In order to get a well-behaved moduli space, one often must first remove some nongeneric, badly-behaved objects. This is true for moduli spaces of sheaves, for example. Sheaves are classified as being stable, semistable, or unstable, and the unstable sheaves are removed from consideration before forming the moduli space.

More precisely, when forming a moduli space we intuitively want to quotient some space, call it $\mathcal{T}$, by the action of some reductive algebraic group $G$. In performing the quotient, we often find that some (nongeneric) points are fixed by a subgroup of $G$ of dimension greater than zero. The orbits of such points are in the closure of other orbits, so if we included them when taking the quotient, the resulting space would not be Hausdorff. To get a well-behaved moduli space we omit them. As these points are typically nongeneric, they are labelled "unstable," and other points are either

\footnotetext{
${ }^{22}$ See appendix C.
} 
"semistable" or just "stable." (Intuitively, a generic perturbation of an unstable point will yield a semistable point, thus the notation.) We shall define the relevant notion of stability (Mumford-Takemoto stability) for sheaves momentarily, and then later in this section will demonstrate explicitly that Mumford-Takemoto stability is realized in our construction of moduli spaces.

Recall a torsion-free sheaf $\mathcal{E}$ on a Kähler variety is said to be MumfordTakemoto stable $[38,39]$ precisely when for any proper coherent subsheaf $\mathcal{F} \subset \mathcal{E}$ such that $0<\operatorname{rank} \mathcal{F}<\operatorname{rank} \mathcal{E}$ and $\mathcal{E} / \mathcal{F}$ is torsion-free, we have

$$
\frac{c_{1}(\mathcal{F}) \cup J^{n-1}}{\operatorname{rank} \mathcal{F}}<\frac{c_{1}(\mathcal{E}) \cup J^{n-1}}{\operatorname{rank} \mathcal{E}}
$$

and semistable if

$$
\frac{c_{1}(\mathcal{F}) \cup J^{n-1}}{\operatorname{rank} \mathcal{F}} \leq \frac{c_{1}(\mathcal{E}) \cup J^{n-1}}{\operatorname{rank} \mathcal{E}}
$$

where $J$ is the Kähler form, and $n$ is the complex dimension of the Kähler variety. The right side of the equation above is often called the slope of $\mathcal{E}$, and is often denoted $\mu(\mathcal{E})$.

Note this means that if $\mathcal{E}$ is an $S L(r, \mathbf{C})$ bundle $\left(c_{1}(\mathcal{E})=0\right)$, then if $\mathcal{E}$ has sections it can be at best semistable, not strictly stable. This is because the section defines a map $\mathcal{O} \rightarrow \mathcal{E}$, so we have a subbundle $\mathcal{F}=\mathcal{O}$ such that $\mu(\mathcal{F})=0=\mu(\mathcal{E})$.

In addition to Mumford-Takemoto stability, there are other (inequivalent) notions of stability for torsion-free coherent sheaves (perhaps most prominently, Gieseker stability). However, these other notions are less relevant for physics than Mumford-Takemoto stability (see section 2), so we will not discuss them here.

\subsection{Moduli Spaces of Equivariant Reflexive Sheaves}

The simplest moduli spaces to study are moduli spaces of equivariant reflexive sheaves, so that is where we shall begin. After describing the construction abstractly, we will work through a specific example in great detail.

In passing, we should clarify a point that may confuse the reader. As the moduli spaces we construct here are moduli spaces of equivariant sheaves (of fixed equivariant structure), the reader may wonder how these are related to moduli spaces of not-necessarily-equivariant sheaves. In fact, the forgetful map that "forgets" the equivariant structure is one-to-one on individual components (of fixed equivariant structure) of a moduli space. More 
precisely [2], if we denote a trivial line bundle with equivariant structure defined by a character $\chi$ by $^{23} \mathcal{O}(\chi)$, then if $\mathcal{E}$ is an indecomposable ${ }^{24}$ sheaf, all equivariant structures on $\mathcal{E}$ can be obtained by tensoring with characters as $\mathcal{E} \otimes \mathcal{O}(\chi)$. (For example, the line bundles $\mathcal{O}\left(D_{x}\right)$ and $\mathcal{O}\left(D_{y}\right)$ on $\mathrm{P}^{2}$ have distinct equivariant structures, but we can get $\mathcal{O}\left(D_{y}\right)$ from $\mathcal{O}\left(D_{x}\right)$ by tensoring with $\mathcal{O}\left(D_{y}-D_{x}\right)$, which is simply the structure sheaf with a nontrivial equivariant structure.) Thus, a moduli space of equivariant sheaves of fixed equivariant structure sits naturally inside a moduli space of not-necessarilyequivariant sheaves.

Subsection 5.2.1 has been described previously by A. A. Klyachko [3] for the special case of equivariant vector bundles on $\mathbf{P}^{2}$.

\subsubsection{Generalities}

By now the reader should be able to make a rough approximation to the form of such moduli spaces. One associates a parabolic subgroup (paired with an ample line bundle on the corresponding partial flag manifold) to each toric divisor, of a general form fixed by the Chern classes. The space of such parabolics on a single toric divisor is a (partial) flag manifold, so the space of all the parabolics is a product of (partial) flag manifolds (one for each toric divisor). Define $\mathcal{T}_{r} \subset \prod_{\alpha} G / P^{\alpha}$, associated to parabolics defining reflexive sheaves of fixed Chern classes. (Although any set of parabolics will define a reflexive sheaf, for certain nongeneric flags the Chern classes will change, and these we shall exclude.) Then, recall that equivalent reflexive sheaves are defined by sets of parabolics differing by an overall $G$-rotation, so loosely speaking the moduli space should look like the quotient of $\mathcal{T}_{r}$ by $G$.

This description is almost correct - the only problem is that we have been too loose in our description of the quotient. The correct quotient to take is not an ordinary quotient, but rather a GIT quotient ${ }^{25}$, in which some "bad" points are omitted. In particular, we do not want to consider all possible equivariant reflexive sheaves, but only those which are Mumford-Takemoto semistable.

Note that to construct $\mathcal{T}_{r}$, we have excluded certain nongeneric points

\footnotetext{
${ }^{23}$ The trivial bundle $\mathcal{O}(\chi)$ has $T$-invariant divisor $D=\sum_{\alpha}\left\langle v_{\alpha}, \chi\right\rangle D_{\alpha}$, where the $v_{\alpha}$ are the fan edges corresponding to the toric divisors $D_{\alpha}$.

${ }^{24}$ For a sheaf to be indecomposable means it cannot be written globally as the direct sum of two subsheaves.

${ }^{25}$ See appendix C.
} 
(whose Chern classes differ) before taking the GIT quotient. The resulting moduli space of equivariant reflexive sheaves is noncompact.

The description of Mumford-Takemoto stability in the equivariant language presented earlier should be clear. Given an equivariant reflexive sheaf $\mathcal{E}$ defined by filtrations $E^{\alpha}(i)$, we say $\mathcal{E}$ is stable if for any nontrivial subspace $^{26} 0 \neq F \subset E$, we have

$$
\begin{aligned}
& \frac{1}{\operatorname{dim} F} \sum_{i \in \mathbf{Z}, \alpha \in|\Sigma|} i \operatorname{dim} F^{[\alpha]}(i)\left(D_{\alpha} \cup J^{n-1}\right) \\
& <\frac{1}{\operatorname{dim} E} \sum_{i \in \mathbf{Z}, \alpha \in|\Sigma|} i \operatorname{dim} E^{[\alpha]}(i)\left(D_{\alpha} \cup J^{n-1}\right)
\end{aligned}
$$

and similarly for semistable reflexive sheaves. We have defined

$$
F^{\alpha}(i)=F \cap E^{\alpha}(i)
$$

and

$$
F^{[\alpha]}(i)=\frac{F^{\alpha}(i)}{F^{\alpha}(i+1)}
$$

The reader may wonder why it is sufficient to check equivariant subsheaves, rather than all subsheaves, in order to test stability. The demonstration of this is quite straightforward [3]. Let $\mathcal{E}$ be an equivariant sheaf, then its Harder-Narasimhan filtration ${ }^{27}$ is also equivariant (in the sense that each subsheaf in the filtration is equivariant). (Were this not the case, then by acting on each element of the filtration with the $\left(\mathbf{C}^{\times}\right)^{n}$ defining the toric variety, we could produce a family of Harder-Narasimhan filtrations of $\mathcal{E}$, but the Harder-Narasimhan filtration is unique.) Now, the Harder-Narasimhan filtration is trivial precisely when $\mathcal{E}$ is semistable, so as all its elements are equivariant, it clearly suffices to check equivariant subsheaves when testing stability.

Now, we want to define a GIT quotient of $\mathcal{T}_{r}$ that acts by first restricting to the Mumford-Takemoto semistable sheaves in $\mathcal{T}_{r}$, then mods out by $G$. In fact, this is a straightforward generalization of a standard example of GIT quotients (see [46, section 4.4] or [47, section 4.6]). The example in the references cited corresponds to a GIT quotient of the space of reflexive sheaves on $\mathbf{P}^{N-1}$, such that on each toric divisor $\alpha, E^{[\alpha]}(i)$ is nontrivial

\footnotetext{
${ }^{26}$ When testing stability of a reflexive sheaf, it is sufficient to check reflexive subsheaves [108], because if $S$ is a destabilizing subsheaf of a reflexive sheaf $\mathcal{E}$, then $S^{\vee \vee}$ is also a destabilizing subsheaf of $\mathcal{E}$, and $S^{\vee \vee}$ is reflexive.

${ }^{27}$ See appendix D.
} 
only for $i=0,1$, i.e., that the parabolic associated to $\alpha$ is maximal. In particular, Mumford-Takemoto stability is realized naturally - the notion of stability defining the GIT quotient (see appendix C) is identical to MumfordTakemoto stability.

The generalization of this example to describe moduli spaces of other reflexive sheaves on arbitrary toric varieties is straightforward. Recall (appendix C) that to construct a GIT quotient of a compact space we must specify an ample, $G$-linearized line bundle on the space. As $\mathcal{T}_{r} \subset \prod_{\alpha} G / P^{\alpha}$, to construct an ample line bundle on $\mathcal{T}_{r}$ we will first specify a line bundle on each factor. The relevant $G$-linearized ample line bundle on $G / P^{\alpha}$ is precisely the ample line bundle $L_{\alpha}$ which together with $P^{\alpha}$ encodes all the information in the filtration (as described in sections 4.1, 4.4).

To take into account the choice of Kähler form $J$, tensor together pullbacks of the $L_{\alpha}$ to $\mathcal{T}_{r}$ weighted by different factors. By working in a dense subset $^{28}$ of the Kähler cone, we can demand that there exist a fixed $\lambda \in \mathbf{R}$ such that for all $\alpha, D_{\alpha} \cup J^{n-1}=\lambda n_{\alpha}$, where $n_{\alpha} \in \mathbf{Z}$.

We can now describe the ample $G$-linearized line bundle on $\mathcal{T}_{r}$ defining the relevant GIT quotient. Let $\pi_{\alpha}: \prod_{\beta} G / P^{\beta} \rightarrow G / P^{\alpha}$ be the canonical projection, then the ample $G$-linearized line bundle $L$ on $\mathcal{T}_{r}$ is

$$
L=\bigotimes_{\alpha} \pi_{\alpha}^{*} L_{\alpha}^{n_{\alpha}}
$$

Note that this is equivalent to composing the Segre embedding of a project of projective spaces with the projective embeddings defined by (tensor powers of) the ample line bundles $L_{\alpha}$.

\subsubsection{A Simple Example}

Before going on, we shall work through a simple example ([46, section 4.4], $[47$, section 4.6]) in detail, in which we shall demonstrate explicitly that the GIT quotient described correctly reproduces Mumford-Takemoto stability. (Our presentation closely follows that of both references. As these methods are new to the physics literature, however, we felt it appropriate to restate the (standard) derivation.) Let us consider equivariant rank- $(n+1)$ reflexive sheaves on $\mathbf{P}^{N-1}$, with equivariant structure such that for all toric divisors $\alpha$

$$
\operatorname{dim} E^{[\alpha]}(i)= \begin{cases}q+1 & i=1 \\ n-q & i=0 \\ 0 & i \neq 0,1\end{cases}
$$

\footnotetext{
${ }^{28}$ More precisely, the subset intersecting Num $\otimes \mathbf{z} \mathbf{Q}$ rather than Num $\otimes \mathbf{z} \mathbf{R}$.
} 
Clearly, each parabolic subgroup $P^{\alpha}$ is maximal, and $G / P^{\alpha}$ is the Grassmannian $G_{q+1}\left(\mathbf{C}^{n+1}\right)$ of $(q+1)$-dimensional subspaces of $\mathbf{C}^{n+1}$ (or, equivalently, the space of $q$-dimensional linear subspaces of $\mathbf{P}^{n}$ ). The ample line bundle $\mathcal{L}$ defined over each $G / P^{\alpha}$ is in fact the very ample line bundle defining the Plücker embedding. We shall assume that the Kähler form $J$ is such that $D_{\alpha} \cup J^{N-2}=1$ for all toric divisors $\alpha$.

Now, we want to describe a GIT quotient that reproduces MumfordTakemoto stability, and in fact this will be quite straightforward.

First, recall the definition of the Plücker embedding

$$
p: G_{q+1}\left(\mathbf{C}^{n+1}\right) \rightarrow \mathbf{P}^{d}
$$

where

$$
d=\left(\begin{array}{c}
n+1 \\
q+1
\end{array}\right)-1
$$

Let $L$ be a $(q+1)$-plane in $\mathbf{C}^{n+1}$, spanned by vectors $v_{0}, \cdots, v_{q} \in \mathbf{C}^{n+1}$, then the Plücker embedding sends this plane to the multivector $v_{0} \wedge \cdots \wedge v_{q}$. Equivalently, if $\left(v_{j 0}, \cdots, v_{j q}\right)$ are the coordinates of the vectors defining $L$, forming a $(q+1) \times(n+1)$ matrix $\Lambda$, then $p_{i_{0} \cdots i_{q}}\left(0 \leq i_{0}<i_{1}<\cdots<i_{q} \leq n\right)$ is the determinant of the $(q+1) \times(q+1)$ submatrix of $\Lambda$ formed with columns $i_{0}, \cdots i_{q}$. Note the $p_{i_{0}, \cdots, i_{q}}$ are homogeneous coordinates of the projective space in which the Grassmannian is embedded.

Now, in order to see explicitly that the choice of polarization ${ }^{29}$ above yields Mumford-Takemoto stability, we shall use the numerical criterion for stability (appendix C). Moreover, as $\mathcal{T}_{r} \subset \prod G_{q+1}\left(\mathbf{C}^{n+1}\right)$, if $x_{\alpha} \in$ $G_{q+1}\left(\mathbf{C}^{n+1}\right) \forall \alpha$, then we know (appendix C)

$$
\mu\left(\left\{x_{\alpha}\right\}, \lambda\right)=\sum_{\alpha} \mu\left(x_{\alpha}, \lambda\right)
$$

so we shall first compute $\mu\left(x_{\alpha}, \lambda\right)$ for a single Grassmannian. (The reader should not confuse the $\mu$ appearing in the numerical criterion of stability with the $\mu$ defining the slope of a bundle. The notation is unfortunate, but standard in the mathematics literature, so we have decided to stay with their conventions.)

The group whose action we wish to $\bmod$ out is $G L(n+1, \mathbf{C})$; however it should be clear that the overall $\mathbf{C}^{\times}$breathing mode will simply act to

\footnotetext{
${ }^{29}$ Recall from appendix $\mathrm{C}$ that when computing the GIT quotient of a variety $\mathcal{T}$ by an algebraic group $G$, the ample line bundle (with $G$-linearization) on $\mathcal{T}$ defining the GIT quotient is known as the polarization.
} 
multiply all the homogeneous coordinates of any projective embedding by an overall factor, which of course is irrelevant. Thus, it is sufficient to consider only $S L(n+1, \mathbf{C})$.

Let $\lambda$ be a one-parameter subgroup of $S L(n+1, \mathbf{C})$ defined by

$$
\lambda(t)=\operatorname{diag}\left(t^{r_{0}}, t^{r_{1}}, \cdots t^{r_{n}}\right)
$$

with $\sum r_{i}=0$, and $r_{0} \geq r_{1} \geq \cdots \geq r_{n}$, with not all the $r_{i}=0$. For given $L \in G_{q+1}\left(\mathbf{C}^{n+1}\right)$, by definition of $\mu$ we have

$$
\mu(L, \lambda)=\max \left\{-\sum_{j=0}^{q} r_{i_{j}} \mid p_{i_{0} \cdots i_{q}}(L) \neq 0\right\}
$$

(Note that this result for $\mu$ depends implicitly upon our choice of projective embedding (here, the Plücker embedding), and so in particular $\mu$ depends on $\mathcal{L}$.)

In order to compute explicitly, we shall work in coordinates. Let $e_{0}, \cdots, e_{n}$ be a basis for $\mathbf{C}^{n+1}$, and define subspaces $L_{i} \in \mathbf{C}^{n+1}$ as

$$
\begin{aligned}
L_{-1} & =\emptyset \\
L_{i} & =\text { subspace generated by images of } e_{0}, \cdots, e_{i} \text { in } \mathbf{C}^{n+1}
\end{aligned}
$$

Note that for any $L \in G_{q+1}\left(\mathbf{C}^{n+1}\right)$ and any integer $j, 0 \leq j \leq q$, there exists a unique integer $\mu_{j}$ such that $\operatorname{dim}\left(L \cap L_{\mu_{j}}\right)=j+1$ and $\operatorname{dim}(L \cap$ $\left.L_{\mu_{j}-1}\right)=j$. Clearly

$$
0 \leq \mu_{0}<\mu_{1}<\cdots<\mu_{q} \leq n
$$

and $L$ is spanned by the rows of a matrix of the form

$$
\left[\begin{array}{cccccccccc}
a_{00} & \cdots & a_{0 \mu_{0}} & 0 & \cdots & & & & \cdots & 0 \\
a_{10} & \cdots & & \cdots & a_{1 \mu_{1}} & 0 & \cdots & & \cdots & 0 \\
\vdots & & & & & & & & \\
a_{q 0} & \cdots & & & & \cdots & a_{q \mu_{q}} & 0 & \cdots & 0
\end{array}\right]
$$

with $a_{j \mu_{j}} \neq 0 \forall j$. In this choice of coordinates, $p_{i_{0} \cdots i_{q}}(L)=0$ if $i_{j}>\mu_{j}$ for any value of $j$ (as, in that event, the $j$ th row would be a linear combination of the previous rows, as all its entries past the $j$ th column would be zero, so the determinant must vanish). It should also be clear that $p_{\mu_{0} \cdots \mu_{q}}(L) \neq 0$, and in fact we can now compute

$$
\mu(L, \lambda)=-\sum_{j=0}^{q} r_{\mu_{j}}
$$




$$
\begin{aligned}
& =-\sum_{i=0}^{n} r_{i}\left[\operatorname{dim}\left(L \cap L_{i}\right)-\operatorname{dim}\left(L \cap L_{i-1}\right)\right] \\
& =-(q+1) r_{n}+\sum_{i=0}^{n-1}\left[\operatorname{dim}\left(L \cap L_{i}\right)\right]\left(r_{i+1}-r_{i}\right)
\end{aligned}
$$

Now, recall that $\mathcal{T}_{r}$ is not a subset of a single Grassmannian, but rather a product of them. As a result, the $\mu$ we need to consider to apply the numerical criterion for stability is

$$
\begin{gathered}
\mu\left(\left\{L^{(1)}, \cdots, L^{(N)}\right\}, \lambda\right)=\sum_{j=1}^{N} \mu\left(L^{(j)}, \lambda\right) \\
=-N(q+1) r_{n}+\sum_{i=0}^{n-1}\left\{\sum_{j=1}^{N}\left[\operatorname{dim}\left(L^{(j)} \cap L_{i}\right)\right]\right\}\left(r_{i+1}-r_{i}\right)
\end{gathered}
$$

In order for a set of subspaces $\left\{L^{(1)}, \cdots, L^{(N)}\right\} \in \mathcal{T}_{r}$ to be stable, we must check that $\mu$ is positive. In fact, as $\mu$ is linear in the $r_{i}$, it will be positive for all allowable $r_{i}$ (meaning, for all one-parameter subgroups of $S L(n+1, \mathbf{C})$ ) precisely when it is positive for the extreme cases

$$
r_{0}=\cdots=r_{p}=n-p, r_{p+1}=\cdots=r_{n}=-(p+1)
$$

for $0 \leq p \leq n+1$.

Thus, $\mu>0$ for all one-parameter subgroups precisely when

$$
N(q+1)(p+1)-(n+1) \sum_{j=1}^{N}\left[\operatorname{dim}\left(L^{(j)} \cap L_{p}\right)\right]>0
$$

for all $0 \leq p \leq n-1$.

Since every $(p+1)$-dimensional subspace of $\mathbf{C}^{n+1}$ is equivalent under $S L(n+1, \mathbf{C})$ to $L_{p}$, we have that a set of flags $\left\{L^{(1)}, \cdots, L^{(N)}\right\} \in \mathcal{T}_{r}$ is stable precisely when for all proper subspaces $L$ of $\mathbf{C}^{n+1}$,

$$
\frac{1}{\operatorname{dim} L} \sum_{j=1}^{N}\left[\operatorname{dim}\left(L^{(j)} \cap L\right)\right]<\frac{1}{n+1}(q+1)(N)
$$

and the reader should immediately recognize this as the statement of MumfordTakemoto stability for the example in question.

If the reader found this derivation overly technical, he may wish to repeat it for the special case that the Grassmannians are all projective spaces, in which case the Plücker embedding is the identity map. 


\subsection{Moduli Spaces of Equivariant Bundles}

These moduli spaces are closely related to the moduli spaces of equivariant reflexive sheaves. Both bundles and reflexive sheaves are described by associating parabolic subgroups of $G$ (paired with ample line bundles on corresponding partial flag manifolds) to each toric divisor. The difference is that in the case of bundles, there is a compatibility condition that must be satisfied (equation (4) for smooth toric varieties).

To construct a moduli space of equivariant bundles, therefore, we proceed almost as in the previous section, except that instead of performing a GIT quotient of $\mathcal{T}_{r} \subset \prod G / P^{\alpha}$, we perform a GIT quotient of the subspace $\mathcal{T}_{b} \subseteq$ $\mathcal{T}_{r}$ consisting of tuples of parabolic subgroups satisfying the compatibility condition. The ample $G$-linearized line bundle defining the appropriate GIT quotient is then just the restriction to $\mathcal{T}_{b}$ of the line bundle constructed in the previous section.

In passing, we should note that in general, $\mathcal{T}_{b}$ will not even be of the same dimension as $\mathcal{T}_{r}$. (In fact, $\mathcal{T}_{b}$ will never be a dense subset of $\mathcal{T}_{r}$ except in the special case $\mathcal{T}_{b}=\mathcal{T}_{r}$.) In many cases, equivariant bundles will be highly nongeneric in a moduli space of equivariant reflexive sheaves.

Let us make a few observations on singularities in the moduli space of equivariant $G$-bundles [106], relevant for generic ${ }^{30}$ Kähler forms. When $G=G L(n, \mathbf{C})$, the moduli space is a GIT quotient of a closed subset of a product of partial flag manifolds, and this will be nonsingular. When $G$ is any other reductive group, the corresponding GIT quotient will have orbifold singularities. For example, when $G$ is (the complexification of) $\operatorname{Spin}(n)$ or $S p(n)$, the moduli space will have $\mathbf{Z}_{2}$ orbifold singularities. For $E_{6}$ or $G_{2}$, the moduli space will have $\mathbf{Z}_{2}$ and $\mathbf{Z}_{3}$ singularities. For $E_{7}$ and $F_{4}$ the moduli space will have $\mathbf{Z}_{2}, \mathbf{Z}_{3}$, and $\mathbf{Z}_{4}$ orbifold singularities. For $E_{8}$ the moduli space will have $\mathbf{Z}_{2}, \mathbf{Z}_{3}, \mathbf{Z}_{4}, \mathbf{Z}_{5}$, and $\mathbf{Z}_{6}$ orbifold singularities. More precisely, for each group the largest singular subvarieties will have the orbifold singularities indicated, but higher codimension substrata may have worse orbifold singularities than indicated above.

In fact we have been rather sloppy. The singularities described above

\footnotetext{
${ }^{30}$ For certain nongeneric Kähler forms, a moduli space of principal $G$-bundles, for any $G$, (and for that matter a moduli space of reflexive sheaves) will have singularities much worse than the orbifold singularities described above. The problem essentially arises when, for certain nongeneric Kähler forms, one gets extra strictly semistable sheaves. For example, we will discuss in section 6 how moduli spaces of rank 2 sheaves on surfaces vary depending upon which subcone of the Kähler cone a Kähler form is in. These moduli spaces are typically birational to one another, and so are extremely singular on a chamber wall.
} 
are singularities expected in a GIT quotient of a product of flag manifolds, but a moduli space of bundles is a subvariety of such a GIT quotient. Just as a hypersurface in a weighted projective space need not have precisely the same orbifold singularities as the ambient weighted projective space, the singularities occurring in a moduli space of bundles may not be precisely those indicated above. However, the singularities listed should be an excellent first approximation.

How are these singularities derived? We will not work through the details (see instead [106]), but the general idea is as follows. In general, when forming the GIT quotient of a product of flag manifolds $K / T$ by $K$, the stabilizers are intersections of tori. Only finite intersections are semistable it can be shown the rest are unstable. Furthermore, these finite intersections turn out to be those subgroups equal to their double centralizers, and it can be shown these are generated by elements corresponding to vertices of the Weyl alcove, whose orders in the adjoint group are the coefficients of the highest root in a basis of simple roots.

In particular, note the singularities above do not quite correspond to singularities of moduli spaces of bundles on elliptic curves [36, 37], One might have expected that the singularities in a moduli space of $G$-bundles on, for example, $\mathbf{P}^{2}$ would also appear in any moduli space of $G$-bundles on a Calabi-Yau hypersurface in $\mathbf{P}^{2}$, but here we see that might not necessarily happen. In fact, there is no reason why it must - in general, the restriction of a stable sheaf to a hypersurface need not be stable.

\subsection{Moduli Spaces of Equivariant Torsion-free Sheaves}

To describe moduli spaces of equivariant torsion-free sheaves involves significantly more technical machinery, and so this has been deferred to a future publication [16].

\subsection{Relations Between Reflexive, Torsion-free Moduli Spaces}

If the reader studies the possible moduli spaces, he will naively come to an apparent contradiction. Let us consider an example to spell out this difficulty. Consider a rank 2 torsion-free sheaf defined over $\mathbf{C}^{2}$. This sheaf is defined by a $\mathbf{Z}^{2}$-graded module, specified by some bifiltration. Suppose 
that the nontrivial part of the bifiltration has the form

\begin{tabular}{c|ccccccc} 
& & -1 & 0 & 1 & 2 & 3 & \\
\hline & & & & $\vdots$ & & & \\
3 & & 0 & 0 & 0 & 0 & 0 & \\
2 & & 1 & 1 & $1^{*}$ & 0 & 0 & \\
1 & $\cdots$ & 2 & 2 & 2 & $1^{*}$ & 0 & $\cdots$ \\
0 & & 2 & 2 & 2 & 1 & 0 & \\
-1 & & 2 & 2 & 2 & 1 & 0 & \\
& & & & $\vdots$ & & &
\end{tabular}

The numbers on the outer edges are the indices of the bifiltration. Those in the middle are the dimensions of elements of the bifiltration. Those marked with a $(*)$ are generators of this module. The numbers indicated are the dimensions of elements of the bifiltration. If the pair of one-dimensional vector spaces marked with a $(*)$ is generic (inside the rank 2 vector space), then their intersection will vanish, and so this bifiltration will correspond to the intersection of two ordinary filtrations, each of which has the form

$$
\operatorname{dim} E(i)= \begin{cases}2 & i \leq 1 \\ 1 & i=2 \\ 0 & i \geq 3\end{cases}
$$

In particular, the torsion-free sheaf will be reflexive, and even a vector bundle.

Now consider the limit that the two one-dimensional vector subspaces marked with a $(*)$ coincide. In this limit the intersection of the two limit filtrations is a bifiltration whose nontrivial components have dimensions

\begin{tabular}{c|ccccccc} 
& & -1 & 0 & 1 & 2 & 3 & \\
\hline & & & & $\vdots$ & & & \\
3 & & 0 & 0 & 0 & 0 & 0 & \\
2 & & 1 & 1 & $1^{*}$ & $1^{\dagger}$ & 0 & \\
1 & $\cdots$ & 2 & 2 & $2^{\dagger}$ & $1^{*}$ & 0 & $\cdots$ \\
0 & & 2 & 2 & 2 & 1 & 0 & \\
-1 & & 2 & 2 & 2 & 1 & 0 & \\
& & & & $\vdots$ & & &
\end{tabular}

Clearly, this bifiltration is not the same as the one indicated in diagram (20). For example, the (one-dimensional) generators are now located in positions marked by $(\dagger)$. In particular, in this limit the torsion-free sheaf indicated in diagram (20) is no longer reflexive. 
The apparent problem arises when we now consider the same limit, but only within the set of data defining a reflexive sheaf. Reflexive sheaves are described only by a set of filtrations on each toric divisor, and in particular in all such limits (in which various vector spaces in filtrations coincide) one still gets a reflexive sheaf. Yet we saw above that when we work in data defining torsion-free sheaves, we recover a sheaf that is torsion-free but specifically not reflexive in this limit!

Thus, the precise sheaf appearing in various limits, depends upon whether we are describing sheaves using only reflexive data or more general torsionfree data.

How can this possibly be consistent? The answer is that in the limit that the two one-dimensional vector subspaces coincide, the Chern classes change $^{31}$. Since we only want to consider moduli spaces of fixed Chern classes, such limiting cases should be omitted. This point has been made obliquely several times previously within this paper, but we felt it sufficiently important to warrant repeating.

These limiting points of reflexive sheaves in which the Chern classes change are excluded from the product of partial flag manifolds before computing any moduli space $\mathcal{M}_{r}$ of equivariant reflexive sheaves as a GIT quotient. As a result, any such moduli space $\mathcal{M}_{r}$ is noncompact. To compactify the moduli space, we must add torsion-free sheaves.

\section{Structure Within The Kähler Cone}

\subsection{Generalities}

Note that Mumford-Takemoto stability depends implicitly upon the choice of Kähler form $[63,64,65,66,67,68,69,70]$. This choice is extremely important - sheaves that are stable with respect to one Kähler form may not be stable with respect to another. In general, for fixed Chern classes a moduli space of sheaves will not have the same form everywhere inside the Kähler cone, but rather will have walls along which extra sheaves become semistable. When these walls are real codimension one in the classical Kähler cone, they stratify the Kähler cone into subcones in each of which the notion of stability

\footnotetext{
${ }^{31}$ To be precise, if the label the toric divisors on $\mathbf{C}^{2}$ as $D_{1}, D_{2}$, then the reflexive sheaf in diagram (20) is precisely $\mathcal{O}\left(D_{1}+2 D_{2}\right) \oplus \mathcal{O}\left(2 D_{1}+D_{2}\right)$ for generic flags, whereas the reflexive sheaf in diagram (21) is precisely $\mathcal{O}\left(D_{1}+D_{2}\right) \oplus \mathcal{O}\left(2 D_{1}+2 D_{2}\right)$. The first Chern classes are identical, but the second Chern classes differ.
} 
is constant. (In fact this phenomenon is quite similar to the behavior of GIT quotients under change of polarization, as discussed in $[55,56]$.)

The most extreme case of this phenomenon, namely when the walls are real codimension one and stratify the Kähler cone into inequivalent subcones, is known to occur for the case of rank 2 sheaves on surfaces. (Other cases are less well understood at present.) In this section we shall make some general remarks on rank $r$ sheaves on algebraic surfaces [80, section 4.C], and indicate when stronger statements can be made by restricting to $r=2$. Again, our remarks are specific to surfaces - for varieties of dimension not equal to two, there are nontrivial differences, which are discussed to some extent in the literature.

Define the discriminant ${ }^{32}$ of a coherent sheaf $\mathcal{E}$ on an algebraic Kähler surface to be

$$
\Delta=2 r c_{2}(\mathcal{E})-(r-1) c_{1}(\mathcal{E})^{2}
$$

where $r$ is the rank of $\mathcal{E}$. It can be shown [80, section 3.4] that when $\mathcal{E}$ is semistable, $\Delta(\mathcal{E}) \geq 0$.

Walls inside the Kähler cone $K$ are specified by divisors $\zeta$ such that $-\frac{r^{2}}{4} \Delta \leq \zeta^{2}<0$. (Not all such divisors define walls along which extra sheaves become semistable; this is a necessary but not sufficient condition.) For a divisor $\zeta$, the corresponding wall is

$$
W_{\zeta}=\{J \in K \mid \zeta \cdot J=0\}
$$

(Note that by the Hodge index theorem, on an algebraic Kähler surface the positive definite part of $H^{1,1}$ is one-dimensional, so the intersection form on $H^{1,1}$ has signature $(+,-, \cdots-)$.) More precisely [80, section 4.C], if $\mathcal{E}$ is a rank $r$ torsion-free coherent sheaf and $\mathcal{E}^{\prime}$ is a subsheaf of rank $r^{\prime}$, with $\mu_{J}(\mathcal{E})=\mu_{J}\left(\mathcal{E}^{\prime}\right)$, then $J$ lies on a wall $W_{\zeta} \operatorname{defined}^{33}$ by $\zeta=r c_{1}\left(\mathcal{E}^{\prime}\right)-r^{\prime} c_{1}(\mathcal{E})$.

\footnotetext{
${ }^{32}$ The discriminant is a special case of a "logarithmic invariant" of a coherent sheaf $\mathcal{E}$. More generally, the logarithmic invariants $\Delta_{i}(\mathcal{E}) \in H^{2 i}(\mathbf{Z})$ are given by

$$
\ln c h(\mathcal{E})=\ln r+\sum_{i=1}(-)^{i+1} \Delta_{i}(\mathcal{E})
$$

where $r$ is the rank of $\mathcal{E}$.

${ }^{33}$ We need to check that $\zeta \cdot J=0$ and $-\frac{r^{2}}{4} \Delta \leq \zeta^{2}<0$. The former is clear. To show the latter, first define $\mathcal{E}^{\prime \prime}=\mathcal{E} / \mathcal{E}^{\prime}$. Note $\mathcal{E}^{\prime \prime}$ is a semistable (as all subsheaves of $\mathcal{E}$ descend to subsheaves of $\mathcal{E} / \mathcal{E}^{\prime}$ ) torsion-free coherent sheaf, of rank $r^{\prime \prime}=r-r^{\prime}$. Use the identity

$$
\Delta(\mathcal{E})-\frac{r}{r^{\prime}} \Delta\left(\mathcal{E}^{\prime}\right)-\frac{r}{r^{\prime \prime}} \Delta\left(\mathcal{E}^{\prime \prime}\right)=-\frac{\zeta^{2}}{r^{\prime} r^{\prime \prime}}
$$

and the fact that $\zeta \cdot J=0$ implies $\zeta^{2} \leq 0$, with equality only when $\zeta=0$, by the Hodge index theorem.
} 
Again, not all divisors $\zeta$ such that $-\frac{r^{2}}{4} \Delta \leq \zeta^{2}<0$ specify walls along which the moduli space changes.

In the special case $r=2$, the precise condition for a divisor $\zeta$ to define a wall along which the moduli space changes is known $[63,64,65,66]$. In addition to the constraint $-\frac{r^{2}}{4} \Delta \leq \zeta^{2}<0$, one must also demand $\zeta-c_{1}=2 F$ for some divisor $F$. For $r=2$, these are the precise conditions for a divisor $\zeta$ to define a wall along which one finds additional strictly semistable sheaves.

Moduli spaces associated to distinct chambers of a Kähler cone are often, but not always, birational to one another. For example it can be shown [80, section 4.C] that if $\Delta \gg 0$, then for any two polarizations $J, J^{\prime}$, the moduli spaces of sheaves of fixed rank $r$ and Chern classes are birational.

In passing we should comment on the physics associated to this phenomenon. First, we should warn that it is possible that this phenomenon might not be seen in heterotic compactifications. Stability is a necessary but not sufficient condition for a consistent compactification, so it is possible (though highly unlikely, in our opinion) that sheaves that are stable in only part of a Kähler cone can not be used in compactifications. Assuming that this does not happen, then we should also remark on Kähler forms in string theory. As is well-known, in string compactifications the Kähler form is complexified, and we expect the imaginary part (the theta angle) will allow us to analytically continue from one moduli space to another, effectively smoothing over the transition.

There is also a closely related topological (rather than algebro-geometric) version of this same phenomenon. See [71, 72] for recent discussions.

\subsection{Application to F Theory}

Suppose we have an elliptic K3 with section. Let $S$ denote the section, and $F$ the (elliptic) fiber, so $S^{2}=-2, F^{2}=0$, and $S \cdot F=1$. Write a divisor $J=a S+b F$, then $J$ is ample precisely when $a>0, b>2 a$. See for example figure 2 .

Clearly by staying sufficiently close to the outer boundaries of the Kähler cone it is possible to never cross a chamber wall. For example, in [29, 30, 31 it is implicitly assumed that the fiber is very small compared to the section. In particular, this assumption means working close to one of the outer boundaries of the Kähler cone, and so the authors of [29, 30,31] never had to worry about crossing a chamber boundary. For the other outer Kähler boundary, we can be slightly more precise. Consider the ample divisor 


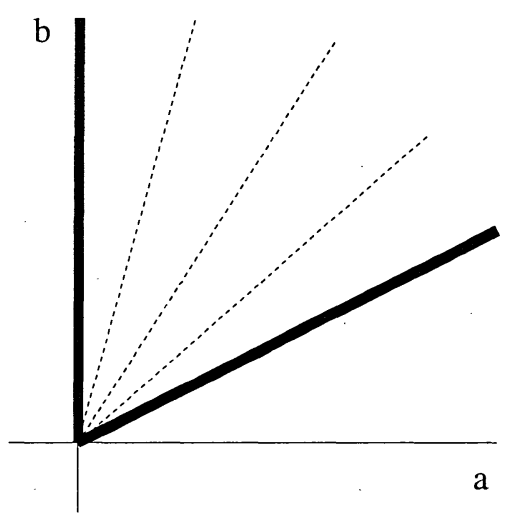

Figure 2: Schematically illustrated is a Kähler cone of a generic elliptic K3 with section. The outer boundaries of the Kähler cone are shown in bold lines, and a few of the chamber boundaries are shown as dotted lines.

$J=S+r F$. According to [63], all such $J$ with $r>1+\left(4 c_{2}-c_{1}^{2}\right) / 2$ are in a single chamber.

For example, consider rank 2 sheaves of $c_{1}=0$ on an elliptic K3 with section. For $c_{2}=4$ and $c_{2}=5$, there are two chambers, with a chamber wall defined by $J=a S+b F$ with $b=3 a$.

Although we can locate the chamber boundaries mathematically, locating them in $\mathrm{F}$ theory is much more difficult. First, F theory does not directly sense the distinction between complex and Kähler structures on the K3, but only the choice of Riemannian metric, which makes the problem of seeing the K3 Kähler cone in F theory somewhat subtle. Secondly, worldsheet instanton corrections will smooth over transitions between chambers when $\alpha^{\prime}>0$, so in order to clearly see a boundary one must go to an entirely classical limit.

\subsection{Kähler Cone Substructure and Equivariant Moduli Spaces}

Let us see explicitly how this phenomenon appears in moduli spaces of equivariant reflexive sheaves. These moduli spaces are constructed as GIT quotients, in which essentially the same phenomena occur under change of polarization. In these moduli spaces we will be able to see the chamber structure explicitly.

Let us consider a specific example, a variation on the example considered in section 5.2.2. Let us work on a Hirzebruch surface $\mathbf{F}_{n}$. A fan for such a 


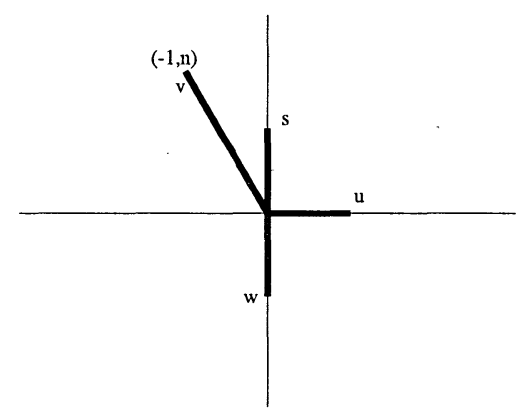

Figure 3: A fan describing the Hirzebruch surface $\mathbf{F}_{n}$ as a toric variety.

surface is shown in figure 3 . In our notation, $D_{u}=\{u=0\}$, for example, and there are the relations $D_{u}=D_{v}, D_{w}=n D_{u}+D_{s} . H^{2}(\mathbf{Z})$ is generated by $D_{u}$, $D_{s}$, and $H^{4}(\mathbf{Z})$ is generated by $D_{u} \cdot D_{s}$, with $D_{u}^{2}=0$ and $D_{s}^{2}=-n D_{u} \cdot D_{s}$.

Our example is defined by filtrations associated to each toric divisor of the form

$$
\operatorname{dim} E^{[\alpha]}(i)= \begin{cases}1 & i=1 \\ r-1 & i=0 \\ 0 & i \neq 0,1\end{cases}
$$

For generic flags the Chern classes of this bundle are $c_{1}=2 D_{s}+(n+2) D_{u}$ and $c_{2}=4 D_{u} \cdot D_{s}$, so $\Delta=8 D_{u} \cdot D_{s}$. Note that $\mathcal{T}_{r} \subset \prod \mathbf{P}^{r-1}$. Expand the Kähler form ${ }^{34}$ as $J=a D_{u}+b D_{s}$, then the Kähler cone is given by $b>0$, $a>n b$, and we have the intersections with divisors

$$
\begin{aligned}
D_{u} \cdot J & =b \\
D_{v} \cdot J & =b \\
D_{w} \cdot J & =a \\
D_{s} \cdot J & =a-n b
\end{aligned}
$$

What chamber structure can we expect in the case $r=2$ ? Using the analysis described earlier, it is easy to show that for $n=1$, the Kähler cone is split into two chambers, with the chamber wall defined by $J=a D_{u}+b D_{s}$ with $2 a=3 b$. For $n=2,3$, there are no nontrivial chamber walls - the only chamber is the entire Kähler cone. We will momentarily check these results by an explicit computation.

For reasons of notation, define $k_{\alpha}=D_{\alpha} \cdot J$. Then, the GIT quotient is defined by the ample $G$-linearized line bundle

$$
\bigotimes_{\alpha} \pi_{\alpha}^{*}\left(\mathcal{O}_{\mathbf{P}^{n}}\left(k_{\alpha}\right)\right)
$$

\footnotetext{
${ }^{34}$ In Num rather than Num $\otimes \mathbf{R}$.
} 
on $\mathcal{T}_{r}$. Note that this bundle is ample precisely when all the $k_{\alpha}>0$, which is true precisely because $J$ lies in the ample cone. In other words, the fact that $J$ defines a nondegenerate Kahler form is intimately connected to the fact that the GIT quotient is well-defined. Now, let $\left\{L^{(\alpha)}\right\} \in \mathcal{T}_{r}$, and it should be clear that this point is Mumford-Takemoto semistable precisely when for all linear subspaces $W \subset \mathbf{P}^{r-1}$,

$$
\frac{1}{\operatorname{dim} W+1} \sum_{\alpha \text { s.t. } L^{(\alpha)} \in W} k_{\alpha} \leq \frac{1}{r} \sum_{\alpha} k_{\alpha}
$$

Now suppose there exists a subspace $W$ such that

$$
\frac{1}{\operatorname{dim} W+1} \sum_{\alpha \text { s.t. } L^{(\alpha)} \in W} k_{\alpha}=\frac{1}{r} \sum_{\alpha} k_{\alpha}
$$

Let us specialize to the case $r=2$, and explicitly reproduce the chamber structure described above. (In fact our calculation is similar to calculations presented in [55].) In this case, in order to get the Chern classes described, one must be careful about the filtrations chosen. In particular, the only times a pair of $L^{(\alpha)}$ can coincide as subspaces of $\mathbf{C}^{2}$ is when the toric divisors do not intersect. In particular, this means that the pair $L^{(u)}, L^{(v)}$ can coincide, as well as the pair $L^{(w)}, L^{(s)}$, but no others.

On $\mathbf{F}_{1}$, the condition for strict semistability is that there exists a subspace $W \subset \mathbf{P}^{1}$ such that

$$
\begin{aligned}
\sum_{\alpha \text { s.t. } L^{(\alpha)} \in W} k_{\alpha} & =\frac{1}{2} \sum_{\alpha} k_{\alpha} \\
& =a+\frac{1}{2} b
\end{aligned}
$$

In the case that $W$ contains only a single $L^{(\alpha)}$, we get no information. Now, the only time a subspace $W$ can contain more than a single $L^{(\alpha)}$ is when the toric divisors are the pair $D_{u}, D_{v}$, or the pair $D_{w}, D_{s}$. In the first case,

$$
\sum_{\alpha \text { s.t. } L^{(\alpha)} \in W} k_{\alpha}=2 b
$$

In the second case,

$$
\sum_{\alpha \text { s.t. } L^{(\alpha)} \in W} k_{\alpha}=2 a-b
$$

and in either event, for strict semistability we immediately derive

$$
a=\frac{3}{2} b
$$


This is precisely the chamber wall expected. Similarly, for $\mathbf{F}_{2}$ and $\mathbf{F}_{3}$, it is easy to show that there are no chamber walls inside the Kähler cone, as claimed earlier. Thus, we can see explicitly the chamber structure predicted by the more abstract analysis given earlier.

\section{Moduli Spaces of Not-necessarily-equivariant Sheaves}

So far in this paper we have primarily studied only equivariant sheaves and their moduli spaces. The reader may well wonder what can be said about moduli spaces of not-necessarily-equivariant sheaves, and how much information about the general case can be obtained by studying only equivariant objects.

In fact, a great deal of information about more general moduli spaces can be gained by studying only the equivariant objects. Full moduli spaces have natural stratifications, the Białynicki-Birula stratifications, in which each stratum is a vector bundle over a moduli space of equivariant sheaves. This is discussed in more detail in subsection 7.1. (This has also been discussed previously in $[1,2,3,4]$.)

In addition, the reader may wonder if any examples of not-necessarilyequivariant moduli spaces are known explicitly. In fact, a few examples are indeed known explicitly, and we examine one in detail in subsection 7.2.

Finally, as an aside, in subsection 7.3 we show how the ADHM construction can be recovered given the results in subsection 7.2, following [75].

The material in this section is not used in other sections, so on a first reading the reader may want to skip to the next section.

In passing, we should clarify a few points that may confuse the reader. First, we have been somewhat sloppy in our use of the word "equivariant." We have implicitly assumed throughout that if a sheaf is equivariant, in our sense of the word, then we are necessarily able to give it an equivariant structure. In fact, this is not quite correct, there is a minor subtlety. Consider for example the tautological bundle on $\mathbf{P}^{1}$. The space $\mathbf{P}^{1}$ can be realized as the coset $S L(2, \mathbf{C}) / B, B$ a Borel subgroup of $S L(2, \mathbf{C})$, so $\mathbf{P}^{1}$ has a natural $S L(2, \mathbf{C})$ action. The center of $S L(2, \mathbf{C})$ acts trivially on $\mathbf{P}^{1}-$ the algebraic torus of which $\mathbf{P}^{1}$ is a compactification is actually

$$
\frac{\text { maximal torus of } S L(2, \mathbf{C})}{\mathbf{Z}_{2}}
$$


The center of $S L(2, \mathrm{C})$ does not act trivially on the total space of the tautological bundle, however - it acts as (-) on the fibers. Thus, the algebraic torus action on $\mathbf{P}^{1}$ does not lift to an action on the tautological bundle, but a finite extension of it does. Throughout this paper, we have assumed that any such extensions are performed whenever needed. In other words, our definition of equivariant sheaf is not rigorous, and in a more mathematical treatment should be replaced with a definition that specifies a lift of the algebraic torus action.

As the moduli spaces we construct here are moduli spaces of equivariant sheaves (of fixed equivariant structure), the reader may wonder how these are related to moduli spaces of not-necessarily-equivariant sheaves. In fact, the forgetful map that "forgets" the equivariant structure is one-to-one on individual components (of fixed equivariant structure) of a moduli space. More precisely [2], if we denote a trivial line bundle with equivariant structure defined by a character $\chi$ by $^{35} \mathcal{O}(\chi)$, then if $\mathcal{E}$ is an indecomposable ${ }^{36}$ sheaf, all equivariant structures on $\mathcal{E}$ can be obtained by tensoring with characters as $\mathcal{E} \otimes \mathcal{O}(\chi)$. (For example, the line bundles $\mathcal{O}\left(D_{x}\right)$ and $\mathcal{O}\left(D_{y}\right)$ on $\mathbf{P}^{2}$ have distinct equivariant structures, but we can get $\mathcal{O}\left(D_{y}\right)$ from $\mathcal{O}\left(D_{x}\right)$ by tensoring with $\mathcal{O}\left(D_{y}-D_{x}\right)$, which is simply the structure sheaf with a nontrivial equivariant structure.) Thus, a moduli space of equivariant sheaves of fixed equivariant structure sits naturally inside a moduli space of not-necessarily-equivariant sheaves.

\subsection{Białynicki-Birula Stratifications}

Any compact variety with a $\mathbf{C}^{\times}$action has a canonical stratification, the Białynicki-Birula stratification [49]. We explain the B-B stratification first and then apply it to moduli spaces of sheaves on toric varieties.

Let $X$ be a projective complex variety with a $\mathbf{C}^{\times}$action. Let $F$ be the set of fixed-point components of this action. For $f \in F$, define the stratum $X_{f}^{0}$ corresponding to $f$ as

$$
X_{f}^{0}=\left\{x \in X \mid \lim _{z \rightarrow 0} z \cdot x \in f\right\}
$$

This limit exists precisely because $X$ is projective. This plainly contains all the points of $f$ and has a natural retraction onto $f$. If $X$ is smooth, then $X_{f}^{0}$

\footnotetext{
${ }^{35}$ In other words, $\mathcal{O}(\chi)$ is the trivial line bundle with equivariant structure defined by the $T$-invariant divisor $D=\sum_{\alpha}\left\langle v_{\alpha}, \chi\right\rangle D_{\alpha}$ where $v_{\alpha}$ is the edge of the fan associated to toric divisor $D_{\alpha}$.

${ }^{36}$ For a sheaf to be indecomposable means it cannot be written globally as the direct sum of two subsheaves.
} 
is in fact a vector bundle over $f[81,82,50]$. The fixed-point components contain much of the information of the variety. For example, the birational geometry and all the Hodge numbers can be computed given those of the fixed-point components [50].

In many cases this stratification can also be understood in terms of Morse theory. If the action of the $U(1)$ inside $\mathbf{C}^{\times}$is Hamiltonian - in particular, if $X$ is smooth (or even just normal) and projective - then the critical points for the Hamiltonian are exactly the fixed points, and the B-B stratum $X_{f}^{0}$ is precisely the points of $X$ that limit to $f$ under gradient ${ }^{37}$ flow (known as the "unstable manifold" of $f$ in mathematics circles).

It is straightforward to determine the rank of a bundle describing a stratum over a component $f$ of $\mathcal{M}^{T}$. Let $\lambda$ be a one-parameter subgroup determining the stratification, then the rank of the bundle over $f$ equals the number of positive weights in the representation of $\lambda$ in a tangent space to any point of $f$.

For example, consider the Riemann sphere $\mathbf{P}^{1}=\mathbf{C} \cup\{\infty\}$. This space is a toric variety and so has a natural action (by multiplication) of $\mathbf{C}^{\times}$, of which it is a compactification. The $\mathbf{C}^{\times}$action has two fixed points: 0 and $\infty$. The action on the tangent space at 0 has weight 1 , and on the tangent space at $\infty$ has weight -1 . We can then view $\mathbf{P}^{1}$ as a disjoint union of $\{0\} \times \mathbf{C}$ and $\{\infty\}-$ so, as promised, $\mathbf{P}^{1}$ looks like a disjoint union of vector bundles over the components of the fixed-point locus.

In the case of a torus action, in principle there are many B-B stratifications, one for each one-dimensional subgroup of the torus. (In fact, even for a one-dimensional torus, there are actually two distinct stratifications, depending upon the isomorphism chosen with $\mathbf{C}^{\times}$.) However, there are only finitely many distinct stratifications $[54,55,56]$. These distinct stratifications can be described pictorially be a fan of some compact toric variety, and we shall denote this fan the stratification fan.

To describe the stratification fan, first we must discuss a fan naturally associated to the moduli space $\mathcal{M}$ of not-necessarily-equivariant bundles. As is well-known $[59,60]$ the image of a moment map associated with a set of commuting circle-generating Hamiltonian actions is a convex polytope. As the algebraic torus acts on $\mathcal{M}$, we can restrict to $S^{1}$ 's to find a set of commuting circle-generating Hamiltonian actions, and so (for a fixed projective embedding of $\mathcal{M}$ ) we can naturally associate a convex polyhedron $\mathcal{P}$ with $\mathcal{M}$.

\footnotetext{
${ }^{37}$ with respect to a $U(1)$-invariant metric
} 
In principle $[59,60]$ this polytope $\mathcal{P}$ is the convex hull of the images (under the moment map) of the fixed points of the circle actions. (Note that sometimes some of the fixed points will lie in the interior of the polytope, but typically they will be vertices of the polytope.) Such polytopes $\mathcal{P}$ may be of use in understanding $(0,2)$ mirror symmetry, as will be discussed later.

Now, given the convex polyhedron $\mathcal{P}$ derived above, it can be shown [54] that the fan describing distinct Białynicki-Birula stratifications of $\mathcal{M}$, is a refinement of the fan associated to $\mathcal{P}$. In particular, we can obtain the stratification fan by adding edges to the fan associated with $\mathcal{P}$, so that $v$ is an edge precisely when $-v$ is an edge. Intuitively this should be sensible. A one-parameter subgroup $\lambda$ borders two stratifications when it has more fixed points than do generic subgroups. In particular, if a one-parameter subgroup $\lambda$ borders two stratifications, then the same should be true of $\lambda^{-1}$. Thus, it should seem intuitively reasonable that $v$ is an edge of the fan precisely when $-v$ is an edge.

We now apply these ideas to normal, projectively embedded moduli spaces of torsion-free sheaves on toric varieties. This should be relatively clear. Recall the torus action on the toric variety induces a torus action on any moduli space of sheaves, as $\mathcal{E} \mapsto t^{*} \mathcal{E}$ for a torus action $t$. The fixed points of this torus action are precisely the equivariant sheaves, which we have studied in great detail earlier in this paper.

\subsection{Monads and Bundles on Projective Spaces}

In this section, we will describe moduli spaces of $S L(r, \mathbf{C})$ bundles on $\mathbf{P}^{2}$. These moduli spaces have been studied in great detail since the early 1970s, and are quite well understood.

The reader who is interested in the $c_{1} \neq 0$ case may wish to consult $[38,79]$.

To what extent can arbitrary bundles on toric varieties be described? It is known $[42,43]$ that any vector bundle $\mathcal{E}$ on $\mathbf{P}^{n}$ can be obtained as the cohomology of a monad ${ }^{38}$

$$
0 \rightarrow \mathcal{A} \rightarrow \mathcal{B} \rightarrow \mathcal{C} \rightarrow 0
$$

where $\mathcal{A}, \mathcal{C}$ are direct sums of line bundles and $\mathcal{B}$ is a vector bundle such that

$$
\begin{array}{lll}
H^{1}(\mathcal{B}(k))=H^{n-1}(\mathcal{B}(k))=0, & k \in \mathbf{Z} \\
H^{i}(\mathcal{B}(k))=H^{i}(\mathcal{E}(k)), & k \in \mathbf{Z}, 1<i<n-1
\end{array}
$$

\footnotetext{
${ }^{38} \mathrm{~A}$ short complex, such that the first and last pairs of maps are exact.
} 
Unfortunately, except for bundles over $\mathbf{P}^{2}$ and $\mathbf{P}^{3}$ the classification of such monads is not well-understood, and so this is not as useful for describing moduli spaces as one would wish.

To begin our discussion of $S L(r, \mathbf{C})$ bundles on $\mathbf{P}^{2}$, we will describe a result due to Beilinson [44]. Let $\mathcal{E}$ be a holomorphic rank $r$ bundle on $\mathbf{P}^{n}$. There are spectral sequences with $E_{1}$ terms

$$
\begin{aligned}
E_{1}^{p, q} & =H^{q}\left(\mathbf{P}^{n}, \mathcal{E}(p)\right) \otimes \Omega^{-p}(-p) \\
E_{1}^{\prime p, q} & =H^{q}\left(\mathbf{P}^{n}, \mathcal{E} \otimes \Omega^{-p}(-p)\right) \otimes \mathcal{O}(p)
\end{aligned}
$$

both of which converge to

$$
\mathcal{E}^{i}= \begin{cases}\mathcal{E} & i=0 \\ 0 & \text { otherwise }\end{cases}
$$

In other words, $E_{\infty}^{p, q}=0$ when $p+q \neq 0$, and $\oplus_{p} E_{\infty}^{-p, p}$ is the associated graded sheaf of a filtration of $\mathcal{E}$, for both spectral sequences. In this notation $\Omega^{p}$ denotes $\wedge^{p} T^{*} \mathbf{P}^{n}$.

In other words, just as Klyachko [1, 2, 3, 4] describes bundles by associating filtrations to divisors, Beilinson describes bundles in terms of spectral sequences. We shall see shortly that in special cases, the spectral sequence simplifies greatly.

Now, let us use Beilinson's result to derive a monad describing $S L(r, \mathbf{C})$ bundles on $\mathbf{P}^{2}$, following [38, section II.3.2]. Let $\mathcal{E}$ be a (properly) stable holomorphic $r$-bundle over $\mathbf{P}^{2}$.

Since $\mathcal{E}$ is properly stable, we can derive a few constraints on certain sheaf cohomology groups. In particular, for $k \geq 0, h^{0}\left(\mathbf{P}^{2}, \mathcal{E}(-k)\right)=0$. If this were not the case, then a section $s$ of $\mathcal{E}(-k)$ would define a map $\mathcal{O} \rightarrow \mathcal{E}(-k)$, and so we would have a map $\mathcal{O}(k) \rightarrow \mathcal{E}$. But then $\mathcal{O}(k)$ would be a subbundle of $\mathcal{E}$, with $\mu(\mathcal{O}(k))=k>\mu(\mathcal{E})=0$, violating the stability condition. Also, by Serre duality, this means $h^{0}\left(\mathbf{P}^{2}, \mathcal{E}^{*}(k)\right)=0$ for $k \geq-3$.

We can also derive a constraint on $c_{2}(\mathcal{E})$ for properly stable bundles on $\mathbf{P}^{2}$. Using Hirzebruch-Riemann-Roch it is easy to show $h^{1}\left(\mathbf{P}^{2}, \mathcal{E}\right)=c_{2}(\mathcal{E})-r$, so clearly $c_{2}(\mathcal{E}) \geq r$.

Apply one of Beilinson's spectral sequences to $\mathcal{E}(-1)$ :

$$
E_{1}^{p, q}=H^{q}\left(\mathbf{P}^{2}, \mathcal{E}(-1) \otimes \Omega^{-p}(-p)\right) \otimes \mathcal{O}(p)
$$


We can show

$$
E_{1}^{0, q}= \begin{cases}H^{1}\left(\mathbf{P}^{2}, \mathcal{E}(-1)\right) \otimes \mathcal{O} & q=1 \\ 0 & q=0,2\end{cases}
$$

By tensoring the short exact sequence

$$
0 \rightarrow \Omega^{1}(1) \rightarrow \mathcal{O}^{3} \rightarrow \mathcal{O}(1) \rightarrow 0
$$

with $\mathcal{E}(-1)$, we can show $h^{0}\left(\mathbf{P}^{2}, \mathcal{E} \otimes \Omega^{1}\right)=0$, and similarly $h^{2}\left(\mathbf{P}^{2}, \mathcal{E} \otimes \Omega^{1}\right)=$ 0 .

Using these results, one can then show

$$
\begin{aligned}
& E_{1}^{-1, q}= \begin{cases}H^{1}\left(\mathbf{P}^{2}, \mathcal{E} \otimes \Omega^{1}\right) \otimes \mathcal{O}(-1) & q=1 \\
0 & q=0,2\end{cases} \\
& E_{1}^{-2, q}= \begin{cases}H^{1}\left(\mathbf{P}^{2}, \mathcal{E}(-2)\right) \otimes \mathcal{O}(-2) & q=1 \\
0 & q=0,2\end{cases}
\end{aligned}
$$

so $E_{\infty}^{p, q}=E_{2}^{p, q}$, and $\mathcal{E}$ is the cohomology of the monad

$$
0 \rightarrow H \otimes \mathcal{O}(-1) \rightarrow K \otimes \mathcal{O} \rightarrow L \otimes \mathcal{O}(1) \rightarrow 0
$$

where

$$
\begin{aligned}
H & =H^{1}\left(\mathbf{P}^{2}, \mathcal{E}(-2)\right) \\
K & =H^{1}\left(\mathbf{P}^{2}, \mathcal{E} \otimes \Omega^{1}\right) \\
L & =H^{1}\left(\mathbf{P}^{2}, \mathcal{E}(-1)\right)
\end{aligned}
$$

For notational convenience define $n=c_{2}(\mathcal{E})$. Using Hirzebruch-RiemannRoch it is easy to show $\operatorname{dim} H=n=\operatorname{dim} L$, and $\operatorname{dim} K=2 n+r$.

It can be shown [38, p. 291] that two monads of the form of equation (22) define the same bundle $\mathcal{E}$ precisely when they differ by the action of $G L(n, \mathbf{C}), G L(2 n+r, \mathbf{C})$, and $G L(n, \mathbf{C})$ on $H, K$, and $L$, respectively.

The moduli space of rank $r$ bundles on $\mathbf{P}^{2}$ can be characterized a little more precisely by the use of Kronecker modules [76, 77, 78], but we will not do so here.

More precise characterizations of the moduli space of rank $r$ bundles on $\mathbf{P}^{2}$ are somewhat difficult to construct except in special cases. For example [38], the moduli space of stable rank 2 bundles on $\mathbf{P}^{2}$ of $c_{1}=0, c_{2}=2$ is 
$\mathbf{P} H^{0}\left(\mathbf{P}^{2 *}, \mathcal{O}(2)\right)=\mathbf{P}^{5}$ (the space of conics on the dual ${ }^{39}$ projective plane), modulo automorphisms of $\mathbf{P}^{2}$. (In fact almost any such conic can be related to almost any other by an automorphism of $\mathbf{P}^{2}$.)

In passing, one might have hoped that the moduli space of bundles on a Calabi-Yau hypersurface were related to the moduli space of bundles on the ambient space, whose top Chern class is minimal. Here, however, we see that this is wrong, as the moduli space of $S L(2, \mathbf{C})$ bundles on $T^{2}$ is $T^{2}$ [85]. (For that matter, the moduli space of flat $S U(2)$ connections on $T^{2}$ is $\mathbf{P}^{1}$.) $\mathbf{P}^{1}$. We will see in section 8 that all stable $S L(2, \mathbf{C})$ bundles on $\mathbf{P}^{2}$ restrict to semistable bundles on $T^{2}$, but there is no map of $\mathbf{P}^{5}$ onto $T^{2}$ (or $\mathbf{P}^{1}$ ), so clearly there are semistable bundles on $T^{2}$ not obtained by restriction from $\mathbf{P}^{2}$.

\subsection{An Aside: The ADHM Construction}

The ADHM construction of instantons on $\mathbf{R}^{4}$ can be derived from the moduli space of bundles on $\mathbf{P}^{2}$ [75]. Intuitively this should be clear: $\mathbf{P}^{2}$ looks like $\mathbf{C}^{2}=\mathbf{R}^{4}$ with a $\mathbf{P}^{1}$ at infinity. To derive the ADHM construction, one simply restricts to those bundles on $\mathbf{P}^{2}$ which are trivial on the line at infinity. (In principle, one can also derive the ADHM construction via Penrose transformations $[61,62]$ from bundles on $\mathbf{P}^{3}$, but we shall not do so here.)

For completeness, we briefly review the derivation of the ADHM construction. In equation (22), label the map $H \otimes \mathcal{O}(-1) \rightarrow K \otimes \mathcal{O}$ by $A$, and the map $K \otimes \mathcal{O} \rightarrow L \otimes \mathcal{O}(1)$ by $B$, and expand

$$
\begin{aligned}
& A=A_{x} x+A_{y} y+A_{z} z \\
& B=B_{x} x+B_{y} y+B_{z} z
\end{aligned}
$$

where $x, y$, and $z$ are homogeneous coordinates on $\mathbf{P}^{2}$.

Now, demanding that the bundle $\mathcal{E}$ be trivial on the line $z=0$ is equivalent to saying that over $z=0, \operatorname{ker} B / \mathrm{im} A$ is a fixed $r$-dimensional subspace. Given that $A$ is one-to-one and $B$ is onto, we can choose bases such that

$$
A_{x}=\left(\begin{array}{c}
I_{n \times n} \\
0_{n \times n} \\
0_{r \times n}
\end{array}\right), A_{y}=\left(\begin{array}{c}
0_{n \times n} \\
I_{n \times n} \\
0_{r \times n}
\end{array}\right), A_{z}=\left(\begin{array}{c}
\alpha_{1} \\
\alpha_{2} \\
a
\end{array}\right)
$$

\footnotetext{
${ }^{39}$ If $V$ is a rank 3 vector space, so $\mathbf{P}^{2}=\mathbf{P} V$, then the dual projective plane is $\mathbf{P}^{2 *}=$ $\mathbf{P} \wedge^{2} V$.
} 
where $I_{n \times n}$ denotes the identity $n \times n$ matrix, and $0_{r \times n}$ the zero $r \times n$ matrix, and

$$
B_{x}=\left(0_{n \times n}, I_{n \times n}, 0_{n \times r}\right), B_{y}=\left(-I_{n \times n}, 0_{n \times n}, 0_{n \times r}\right), B_{z}=\left(-\alpha_{2}, \alpha_{1}, b\right)
$$

The choice of bases above reduces the original $G L(n, \mathbf{C}), G L(2 n+r, \mathbf{C})$, $G L(n, \mathbf{C})$ symmetries to $G L(n, \mathbf{C})$ and $G L(r, \mathbf{C})$. However, the action of $G L(r, \mathbf{C})$ acts as a gauge transformation on the $\mathbf{P}^{1}$ at infinity, and in constructing the moduli space of instantons on $\mathbf{R}^{4}$ we restrict to gauge transformations which are trivial at infinity, so for the purposes of deriving the ADHM construction we have only a $G L(n, \mathbf{C})$ symmetry left to mod out.

The constraint $B A=0$ reduces to $B_{z} A_{z}=0 \Leftrightarrow\left[\alpha_{1}, \alpha_{2}\right]+b a=0$.

In particular, the moduli space of instantons on $\mathbf{R}^{4}$ can now be described as the quotient of the set of matrices $\left(\alpha_{1}, \alpha_{2}, a, b\right)$ satisfying

$$
\begin{aligned}
& \text { (1) }\left[\alpha_{1}, \alpha_{2}\right]+b a=0 \\
& \text { (2) } A \text { is one-to-one, } B \text { is onto }
\end{aligned}
$$

by the action of $G L(n, \mathbf{C})$ given by

$$
\begin{aligned}
\alpha_{i} & \rightarrow p \alpha_{i} p^{-1} \\
a & \rightarrow a p^{-1} \\
b & \rightarrow p b
\end{aligned}
$$

where $p \in G L(n, \mathbf{C})$.

Even more succinctly, the moduli space of instantons on $\mathbf{R}^{4}$ is the GIT quotient of the set of matrices $\left(\alpha_{1}, \alpha_{2}, a, b\right)$ satisfying

$$
\left[\alpha_{1}, \alpha_{2}\right]+b a=0
$$

by the action of $G L(n, \mathbf{C})$ indicated. With additional work, it can be shown this is equivalent to the symplectic quotient usually mentioned in the physics literature.

\section{Restriction to Hypersurfaces}

So far in this paper we have discussed sheaves on toric varieties. However, in order to get valid heterotic compactifications, we must restrict to CalabiYau hypersurfaces (or, more generally, Calabi-Yau complete intersections) in these toric varieties. 
In general, the restriction of a (semi)stable sheaf on a toric variety to a Calabi-Yau hypersurface need not be (semi)stable (with respect to the restriction of the Kähler form). However, there do exist conditions under which the restriction is known to necessarily be (semi)stable. There are two sets of results in this matter known in the mathematics literature, which we shall outline below.

Let $X$ be an $n$-dimensional normal projective variety, $L$ a very ample line bundle on $X$ defining Mumford-Takemoto stability, and let $\mathcal{E}$ be a semistable torsion-free sheaf of rank $r$ on $X$. Consider restricting the sheaf $\mathcal{E}$ to a generic hypersurface $H$ in the linear system $\left|L^{d}\right|$, with $d>0$.

The first result, due originally to Maruyama [74, 86, 73], is that the restriction of $\mathcal{E}$ to $H$ is semistable for arbitrary $d$ if $r<n$.

The second result, due to Flenner [87], says that the restriction of $\mathcal{E}$ to $H$ is semistable if

$$
\frac{\left(\begin{array}{c}
n+d \\
d
\end{array}\right)-d-1}{d}>\mu(L) \max \left\{\frac{r^{2}-1}{4}, 1\right\}
$$

where $\mu(L)$ is the Mumford-Takemoto slope of $L$, namely $c_{1}(L)^{n}$.

For example, Flenner's result (23) says that for a Calabi-Yau hypersurface in $\mathbf{P}^{2}$, with very ample line bundle $L=\mathcal{O}(1)$, the restriction of $\mathcal{E}$ is semistable if $r \leq 2$. For Calabi-Yau hypersurfaces in $\mathbf{P}^{3}$, the restriction of $\mathcal{E}$ is semistable if $r \leq 5$. For Calabi-Yau hypersurfaces in $\mathbf{P}^{4}$, the restriction of $\mathcal{E}$ is semistable if $r \leq 9$.

Flenner's result is a refinement of a slightly older result often cited in the mathematics literature, which we shall describe here for completeness. The older result is due to Mehta and Ramanathan [88], and says that for fixed semistable $\mathcal{E}$, there exists an integer $d_{0}$ such that for generic hyperplanes $H \in\left|L^{d}\right|, d \geq d_{0}$, the restriction of $\mathcal{E}$ to $H$ is semistable. Unfortunately, the integer $d_{0}$ depends very much upon the precise sheaf $\mathcal{E}$, not just numerical invariants such as its Chern classes. (A very slight refinement of their result also exists [89], which concerns whether the restriction of a stable sheaf is stable.)

Unfortunately all of these results are only valid for very specific Kähler forms. It would be very interesting to have analogous results for more general Kähler forms, but to our knowledge the mathematics community has not yet been so obliging. 


\section{Distler, Kachru's (0,2) Models}

\subsection{Review}

In addition to the recent description of vector bundles given by Friedman, Morgan, Witten [29, 30, 32, 33], there is another description of vector bundles $^{40}$ due originally to Maruyama [41] and generalized in work of Distler, Greene [20] and Distler, Kachru [17].

A Distler-Kachru model is a conformal field theory with global $(0,2)$ supersymmetry constructed as the IR limit of a gauged linear sigma model [21]. The linear sigma model in question describes a Calabi-Yau hypersurface in a toric variety. A sheaf $\mathcal{E}$ lies over the ambient toric variety, and $\mathcal{E}$ is often $^{41}$ specified by a short exact sequence of the form

$$
0 \rightarrow \mathcal{E} \rightarrow \oplus \mathcal{O}\left(n_{a}\right) \rightarrow \oplus \mathcal{O}\left(m_{i}\right) \rightarrow 0
$$

The sheaf over the hypersurface is simply the restriction of $\mathcal{E}{ }^{42}$

Some sheaves over the hypersurface in the toric variety - even some line bundles - may be unobtainable as restrictions of such sheaves over the toric ambient space. This is essentially because a single toric divisor can sometimes intersect a hypersurface more than once, so a single divisor in the ambient space can become multiple divisors on a complete intersection [25]. Moreover, it is not obvious that all bundles over the ambient toric variety can be described as kernels of short exact sequences. (Admittedly though for sheaves on projective toric varieties, the results described at the beginning of section 7.2 make this seem not completely unreasonable.)

Typically Distler-Kachru models are assumed to describe bundles whose structure group is $G L(n, \mathbf{C})$, though it has been observed in [15] that more

\footnotetext{
${ }^{40}$ Actually, Distler, Kachru [17] are more general and describe torsion-free sheaves, not just bundles.

${ }^{41}$ Either by a short exact sequence as shown, or as the cohomology of a monad, which is a short complex such that the first and last pairs of maps are exact. The monads of [17] are typically only defined over complete intersections in toric varieties, not over the entire ambient space. We will not consider such monads in this paper.

${ }^{42}$ In fact, we should be slightly more careful. Let $\iota: Y \hookrightarrow X$ denote the inclusion morphism mapping a Calabi-Yau hypersurface $Y$ into the ambient toric variety $X$. Strictly speaking, given a sheaf $\mathcal{E}$ defined over $X$ by the short exact sequence shown, when we restrict to the Calabi-Yau $Y$ we recover

$$
\cdots \rightarrow \operatorname{Tor}_{1}^{\mathcal{O}_{Y}}\left(\oplus \mathcal{O}\left(m_{i}\right), \mathcal{O}_{Y}\right) \rightarrow \iota^{*} \mathcal{E} \rightarrow \iota^{*} \oplus \mathcal{O}\left(n_{a}\right) \rightarrow \iota^{*} \oplus \mathcal{O}\left(m_{i}\right) \rightarrow 0
$$
}

In reasonably nice cases, however, $\operatorname{Tor}_{1}^{\mathcal{O}_{Y}}\left(\oplus \mathcal{O}\left(m_{i}\right), \mathcal{O}_{Y}\right)=0$, so we recover the naive short exact sequence. 
general structure groups can be obtained in principle, as special cases of $G L(n, \mathbf{C})$ structure groups. One of the advantages to Klyachko's approach is that we can describe bundles of arbitrary structure group with equal facility.

\subsection{Application to Distler-Kachru Models}

Recall in [17] sheaves $\mathcal{E}$ over toric varieties are often specified as the kernel of a short exact sequence

$$
0 \rightarrow \mathcal{E} \rightarrow \oplus \mathcal{O}\left(n_{a}\right) \rightarrow \oplus \mathcal{O}\left(m_{i}\right) \rightarrow 0
$$

Note that such sheaves $\mathcal{E}$ are not usually equivariant (as the maps are not).

As noted earlier, the moduli space $\mathcal{M}$ of torsion-free sheaves has a stratification, in which each stratum looks like a vector bundle over a component of $\mathcal{M}^{T}$. Thus, we shall begin by describing one way to construct an equivariant sheaf associated with a family of sheaves specified as above. Unfortunately it will usually be the case that our recipe will yield a badly behaved result - it seems at least naively that most $(0,2)$ models of Distler, Kachru do not have equivariant limits.

First, for each line bundle, pick a specific equivariant structure - in other words, write $\mathcal{O}(D)=\mathcal{O}\left(\sum a_{\alpha} D_{\alpha}\right)$, for some $\sum a_{\alpha} D_{\alpha}$ in the linear equivalence class of $D$. (In this notation, each $D_{\alpha}$ is a toric divisor.) In fact, each equivariant line bundle is uniquely written as such a sum, if an equivariant structure is taken into account [83]. Note that divisors differing by linear equivalence define line bundles with distinct equivariant structures, so it is necessary to fix specific divisors.

Let $x_{\alpha}$ denote the homogeneous coordinate corresponding to the toric divisor $D_{\alpha}$ [12]. Then the unique (up to rescalings) equivariant map $\mathcal{O}\left(\sum a_{\alpha} D_{\alpha}\right) \rightarrow$ $\mathcal{O}\left(\sum b_{\alpha} D_{\alpha}\right)$ consists of multiplying sections of $\mathcal{O}\left(\sum a_{\alpha} D_{\alpha}\right)$ by the monomial $x_{0}^{b_{0}-a_{0}} x_{1}^{b_{1}-a_{1}} \cdots x_{k}^{b_{k}-a_{k}}$, up to a scale factor.

This constraint can be made somewhat more intuitive as follows. In terms of a homogeneous coordinate description of a toric variety, the algebraic torus appears as rescalings of the individual homogeneous coordinates by $\mathbf{C}^{\times}$'s, modulo the $\mathbf{C}^{\times}$'s one mods out to recover the toric variety. Suppose that one of the maps $\mathcal{O}\left(\sum a_{\alpha} D_{\alpha}\right) \rightarrow \mathcal{O}\left(\sum b_{\alpha} D_{\alpha}\right)$ was a polynomial rather than a monomial, then it should be clear that under the algebraic torus action the coefficients of each monomial in the polynomial would vary, independently of one another. Clearly, when the maps are polynomials, the algebraic torus action deforms $\mathcal{E}$ - a necessary (but not sufficient) condition 


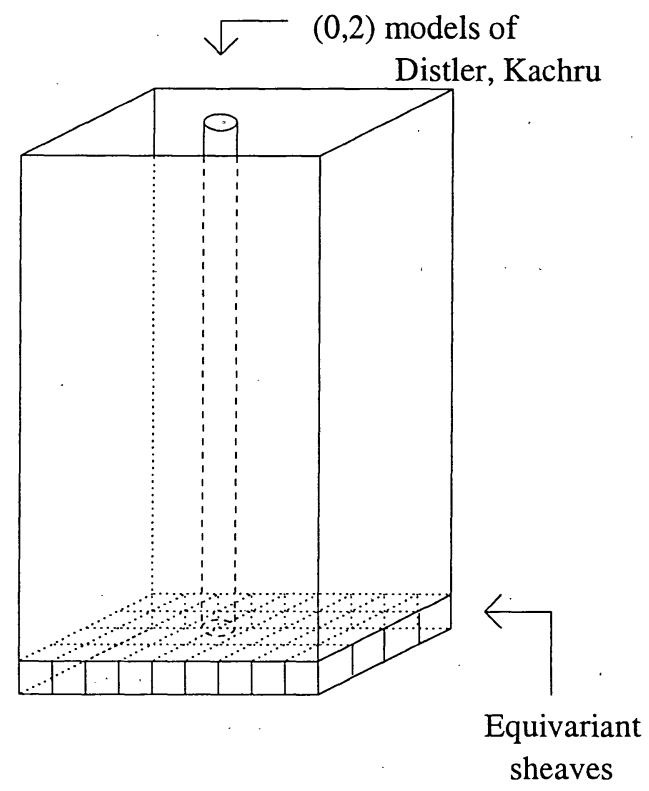

Figure 4: Schematically illustrated is a stratum of a moduli space of sheaves of fixed Chern classes on a toric variety. Equivariant sheaves are located in the shaded region at the bottom, and the region described by a DistlerKachru model is contained in the cylindrical region.

for $\mathcal{E}$ to be invariant under the algebraic torus is for each polynomial to be a monomial.

The sheaf $\mathcal{E}$ defined by these choices of equivariant structures and maps is an equivariant torsion-free sheaf.

Note that we can deform $\mathcal{E}$ equivariantly by varying scale factors in front of the monomials defining the maps $\oplus \mathcal{O}\left(n_{a}\right) \rightarrow \oplus \mathcal{O}\left(m_{i}\right)$. These scale factors maps out a Grassmannian, after removing points at which the scale factors no longer define an exact sequence (i.e., if the map drops rank) and modding out by rescalings of the homogeneous coordinates. This projective space is, in general, a subspace of the moduli space of equivariant torsion-free sheaves.

In figure 4 we have schematically drawn a picture of a stratum of a moduli space of sheaves of fixed Chern classes on some toric variety. The point it is meant to illustrate is that Distler-Kachru models and the equivariant sheaves discussed here scan different parts of the moduli space, and neither is contained within the other. In fact, the figure shows the two subspaces as intersecting, though in fact that seems to be quite rare - most Distler-Kachru models do not seem to have well-behaved equivariant limits. 
By deforming the maps $\oplus \mathcal{O}\left(n_{a}\right) \rightarrow \oplus \mathcal{O}\left(m_{i}\right)$ to more generic polynomials, we can also study non-equivariant sheaves.

In passing, we feel we should make an important point which has long been overlooked in the (physics) literature on heterotic compactifications. For some coherent sheaf $\mathcal{E}$, its deformations are parametrized by elements of (global) $\operatorname{Ext}^{1}(\mathcal{E}, \mathcal{E})$, which gets contributions from $\operatorname{both}^{43} H^{1}(H o m(\mathcal{E}, \mathcal{E}))$ and also $H^{0}\left(\operatorname{Ext}^{1}(\mathcal{E}, \mathcal{E})\right)$. In the special case that $\mathcal{E}$ is locally free (meaning, is a bundle), it is true that (global) $\operatorname{Ext}^{1}(\mathcal{E}, \mathcal{E})=H^{1}(\operatorname{Hom}(\mathcal{E}, \mathcal{E}))$, but more generally the contribution from $H^{0}\left(E x t^{1}(\mathcal{E}, \mathcal{E})\right)$ will be nonzero. This contribution has long been overlooked. Assuming that the additional directions in the moduli space are not lifted by quantum corrections, this should be a source of additional marginal operators overlooked in heterotic nonlinear sigma models. (In non-geometric models, such as heterotic LandauGinzburg orbifolds, marginal operators morally corresponding to elements of $H^{0}\left(E x t^{1}(\mathcal{E}, \mathcal{E})\right)$ presumably appear in twisted sectors.) In addition, other massless modes, traditionally counted by sheaf cohomology groups, are correctly counted with Ext groups [14, 91].

\section{$10(0,2)$ Mirror Symmetry}

For type II compactifications there is a well-known worldsheet duality called mirror symmetry. In mirror symmetry two distinct Calabi-Yaus (a "mirror pair") are described by the same conformal field theory.

Many people have conjectured that there exists a heterotic version of the same phenomenon, known as " $(0,2)$ mirror symmetry." The most conservative potential $(0,2)$ mirror symmetry is that there exist pairs of Calabi-Yaus and bundles that are described by the same conformal field theory. In passing, however, we should note that $(0,2)$ mirror symmetry may do far more. Unlike ordinary mirror symmetry, $(0,2)$ mirror symmetry is not constrained to be a single $\mathbf{Z}_{2}$ involution of the moduli space. For example, there may be multiple distinct $(0,2)$ mirror symmetries acting on the moduli space. Some of them might, for example, say that triples of heterotic compactifications are described by the same conformal field theory.

To date, the only hypothesized examples of $(0,2)$ mirrors have been constructed either as orbifolds [92, 93, 94] of Distler-Kachru models, or via

\footnotetext{
${ }^{43} \operatorname{Hom}(\mathcal{E}, \mathcal{E})=E n d \mathcal{E} \oplus \mathcal{O}$, so on a simply-connected Calabi-Yau, $H^{1}(\operatorname{Hom}(\mathcal{E}, \mathcal{E}))=$ $H^{1}(E n d \mathcal{E})$ Here End refers to traceless endomorphisms, as is standard in the physics literature.
} 
WZW models. No analogue of Batyrev's construction [90] is yet known for $(0,2)$ mirror symmetry.

One might ask whether equivariant sheaves are relevant to the $(0,2)$ generalization of mirror symmetry. In fact, we shall conjecture that there exists a $(0,2)$ mirror symmetry that at least sometimes carries (restrictions to Calabi-Yaus of) equivariant sheaves to other (restrictions to Calabi-Yaus of) equivariant sheaves.

A priori, this would not be expected. In principle, given some sheaf $\mathcal{E}$ over a Calabi-Yau hypersurface, it should be extremely difficult to determine if $\mathcal{E}$ is the restriction of an equivariant sheaf to the hypersurface. Thus, a priori, one might be surprised if some $(0,2)$ generalization of mirror symmetry relates sheaves obtained by restriction of equivariant sheaves. Although such a result is naively surprising, it is completely consistent with what we know about ordinary mirror symmetry, in which analogous surprises are well-known to occur. We will give two rather weak bits of evidence for our conjecture.

First, for at least some $(0,2)$ mirrors constructed as orbifolds of DistlerKachru models, it is easy to show that formally the mirror of the restriction of an equivariant sheaf, is the restriction of another equivariant sheaf.

We shall consider a very simple example just to illustrate the point. Our example is an equivariant sheaf on $\mathbf{P}^{2}$ constructed as the kernel of a short exact sequence

$$
0 \longrightarrow \mathcal{E} \longrightarrow \mathcal{O}\left(D_{x}\right) \oplus \mathcal{O}\left(D_{y}\right) \oplus \mathcal{O}\left(D_{z}\right) \stackrel{F_{a}}{\longrightarrow} \mathcal{O}\left(D_{x}+D_{y}+D_{z}\right) \longrightarrow 0
$$

where

$$
\begin{aligned}
& F_{x} \propto y z \\
& F_{y} \propto x z \\
& F_{z} \propto x y
\end{aligned}
$$

(In our notation, $D_{x}=\{x=0\}$. Note we have explicitly stated the equivariant structure on each line bundle above.) The (restriction of the) sheaf $\mathcal{E}$ defined above is closely related to a deformation of the tangent bundle of $T^{2}$. As discussed in [22] such a Distler-Kachru model suffers from certain poorly understood anomalies that prevent it from being used as a string compactification; however, it will serve admirably to illustrate the general idea.

To construct the orbifold mirror to this equivariant Distler-Kachru model, 
we mod out by a $\mathbf{Z}_{3}$ symmetry that acts on the Calabi-Yau as

$$
x \rightarrow x, y \rightarrow \alpha y, z \rightarrow \alpha^{2} z
$$

where $\alpha$ is a cube root of unity, and on the $\mathcal{O}(1)^{3}$ as

$$
\Lambda_{1} \rightarrow \Lambda_{1}, \Lambda_{2} \rightarrow \alpha \Lambda_{2}, \Lambda_{3} \rightarrow \alpha^{2} \Lambda_{3}
$$

(specifying an action on $\mathcal{O}(1)^{3}$ is sufficient to specify an action on the bundle). Now, given a Distler-Kachru model defined by a sheaf $\mathcal{E}$ of the form

$$
0 \longrightarrow \mathcal{E} \longrightarrow \bigoplus_{a} \mathcal{O}\left(n_{a}\right) \stackrel{F_{a}}{\longrightarrow} \mathcal{O}(m) \longrightarrow 0
$$

when orbifolding the $(0,2)$ model we must restrict to maps $F_{a}$ such that the lagrangian of the $(0,2)$ model is invariant under the orbifold symmetry. It is straightforward to check that in our example above the maps $F_{x}, F_{y}$, and $F_{z}$ (together with an appropriate choice of complex structure for the hypersurface) yield a Lagrangian invariant under the symmetry in equation (24).

Thus, in our example, the orbifold construction of $(0,2)$ mirror symmetry formally appears to carry (restrictions to hypersurfaces of) equivariant sheaves to (restrictions to hypersurfaces of) equivariant sheaves.

Our second bit of evidence for our conjecture comes from studying a special limit of ordinary mirror symmetry. Consider a limit of a mirror pair of Calabi-Yaus $X, Y$ in which all worldsheet instanton corrections on either side are suppressed to zero - in other words, consider the large Kähler modulus and large complex structure limit. In this limit the Calabi-Yau degenerates, typically in such a way that its tangent bundle becomes (stably equivalent to) the restriction of an equivariant sheaf. Ordinary mirror symmetry takes such a degenerate Calabi-Yau, into another similarly degenerate Calabi-Yau. In other words, in this special limit, ordinary mirror symmetry maps (restrictions of) equvariant sheaves to (restrictions of) equivariant sheaves.

If our conjecture is correct, it may give us information about the action of $(0,2)$ mirror symmetry on a moduli space of not-necessarily-equivariant sheaves. In particular, if we associate a polygon to each such moduli space as in section 7.1 , then this suggests that $(0,2)$ mirror symmetry may map the vertices of the polygons into each other.

If our conjecture is correct - if there exists a $(0,2)$ mirror symmetry mapping equivariant sheaves to equivariant sheaves - then in principle it should be possible to at least find a mirror map precisely relating moduli of equivariant sheaves. Unfortunately we have not yet been able to do so. 


\section{Conclusions}

In this paper we have presented an inherently toric description of a special class of sheaves on toric varieties, something that has long been considered extremely desirable. We have stated relevant results on when the restriction of sheaves to Calabi-Yau hypersurfaces are stable, a necessary (but not sufficient) condition for a consistent heterotic compactification. We have described nontrivial substructure in the classical Kähler cone. Finally, we have made some basic attempts to apply this technology to the study of $(0,2)$ mirror symmetry.

We believe that one of the most promising future directions for this work lies in building a nontrivial understanding of the $(0,2)$ generalization of mirror symmetry. Such insights would vastly improve our knowledge of heterotic compactifications, and surely lead to improvements in our understanding of nonperturbative effects in heterotic string theory.

In passing we should mention two other recent developments that may also lead to an understanding of $(0,2)$ mirror symmetry. First, in addition to the "traditional" construction of mirror symmetric pairs à la Batyrev [90], there has recently been an attempt to understand mirror symmetry from a very different perspective $[95,96,97,98]$, as a sort of T-duality on the fibers of a special Lagrangian fibration of a Calabi-Yau. At the moment this approach to understanding ordinary mirror symmetry is still in its infancy, but it holds great promise for giving insight into ordinary mirror symmetry and perhaps also $(0,2)$ mirror symmetry.

There have also recently been some very interesting attempts by mathematicians to apply technology originally designed for real 3- and 4-manifolds to Calabi-Yau 3- and 4-folds with stable holomorphic sheaves [99, 100, 101]. Their holomorphic versions of the Casson, Floer, and Donaldson invariants may well turn out to be very important in understanding heterotic compactifications.

Finally, as this paper was being finished, a paper appeared [102] which claims to give an intuitive understanding of ordinary mirror symmetry as a particular form of T-duality. It may be possible to apply their ideas to our technology to gain insight into $(0,2)$ mirror symmetry; work on this is in progress [16]. 


\section{Acknowledgements}

The authors would like to thank R. Friedman, T. Gomez, S. Kachru, J. Morgan, E. Silverstein, K. Uhlenbeck, and E. Witten for useful discussions. We would also like to thank A. A. Klyachko for supplying us with the preprints $[3,4]$ and for creating a beautiful mathematical theory.

\section{A Algebraic Groups}

An algebraic group is a group that also happens to be a variety, just as a Lie group is a group that also happens to be a manifold. Put another way, algebraic groups are to algebraic geometry what Lie groups are to differential geometry. In what follows we shall only discuss connected, complex subgroups of $G L(n, \mathbf{C})$. For more information, see for example [51, 52, 53].

Reductive algebraic groups are algebraic groups which are the complexification of a compact Lie group. Put another way, a reductive algebraic group is an algebraic group such that its maximal compact subgroup has half the $\mathbf{R}$ dimension of the algebraic group itself. Not all algebraic groups are reductive: a basic example is $\mathbf{C}$ under + . An algebraic group is called solvable if its maximal compact subgroup is a torus. An algebraic group is called unipotent if its maximal compact subgroup is a point (meaning, the group is contractible). Unipotent algebraic groups have the property that their Lie algebras can be represented by nilpotent matrices. A Borel subgroup of a reductive group $G$ is a maximal solvable subgroup. All Borel subgroups of $G$ are conjugate. A parabolic subgroup is a subgroup containing a Borel subgroup.

For example, for the algebraic group $G L(n, \mathbf{C})$, the subgroup consisting of all upper-triangular matrices is a Borel subgroup. A subgroup consisting of block-upper-triangular matrices is a parabolic subgroup. Parabolic subgroups of an algebraic group (other than the group itself) are not reductive.

Borel and parabolic subgroups can be understood efficiently in terms of the Lie algebra of an algebraic group $G$. For some choice of Cartan subalgebra and Weyl chamber, a Borel subgroup is a subgroup generated by the Cartan subalgebra and all the positive roots. To get a parabolic subgroup, add some of the negative simple roots and what they generate. In our conventions, both a Borel and $G$ itself are considered special cases of parabolic subgroups. Maximal parabolic subgroups $(\neq G)$ are in one-to-one correspondence with points on the Dynkin diagram. 
Parabolic subgroups have a (non-canonical) splitting known as the Levi decomposition. More precisely, any parabolic subgroup $P$ can be written $P=L U$ where $U$ is a unipotent subgroup, and $L$ (known as the Levi factor) is reductive. ( $U$ is unique, but $L$ is only unique up to conjugation by elements of $U$.)

A useful fact is that the intersection of any two parabolic subgroups contains a maximal torus of $G$. (Remember that any two parabolic subgroups both contain a Borel subgroup, but not necessarily the same Borel subgroup.)

(Partial) flag manifolds can be obtained as quotients of $G L(n, \mathbf{C})$ by parabolic subgroups. This is closely related to a description of flag manifolds in terms of Lie groups. For example, the Grassmannian of $k$ planes in $\mathbf{C}^{n}$ is

$$
\begin{aligned}
G_{k}\left(\mathbf{C}^{n}\right) & =G L(n, \mathbf{C}) / P_{k} \\
& =\frac{U(n)}{U(k) \times U(n-k)}
\end{aligned}
$$

where the $P_{k}$ are maximal parabolics of $G L(n, \mathbf{C})$. The $P_{k}$ are very closely related to $U(k) \times U(n-k)$ - the Levi factors of the $P_{k}$ are $G L(k, \mathbf{C}) \times G L(n-$ $k, \mathbf{C})$, the complexification of $U(k) \times U(n-k)$. One can define "generalized flag manifolds," which are coset spaces $G / P, P$ a parabolic subgroup of $G$, for arbitrary $G$.

Fix a Borel subgroup $B$ of $G$, and let $T$ be a maximal torus in $B \subset G$. Define $N=N_{G}(T)=\left\{x \in G \mid x T x^{-1}=T\right\}$, the normalizer of $T$ in $G$. The Weyl group is $W=N / T$. The Bruhat decomposition of $G$ is then

$$
G=\bigcup_{\sigma \in W} B \widetilde{\sigma} B
$$

where the union is a disjoint union, and each $\widetilde{\sigma}$ is a representative of a coset $\sigma \in N / T$. In other words, for each element $g \in G$ there is a unique $\sigma \in W$ such that $g$ lies inside $B \widetilde{\sigma} B$. The reader may enjoy proving this for $G L(n, \mathbf{C})$, taking the $\{\tilde{\sigma}\}$ to be permutation matrices (a single 1 in each row and column). In this case the Bruhat decomposition says that any invertible matrix can be brought to a unique permutation matrix using upward row and rightward column operations.

\section{B Sheaf Classifications}

A few general remarks are in order concerning sheaves. By a locally free sheaf we mean a sheaf associated to a vector bundle - the sheaf of local 
sections of the vector bundle. A torsion-free sheaf is a sheaf which, on a smooth variety, is locally free except along a codimension two subvariety so (on a smooth variety) a torsion-free sheaf can be thought of as a vector bundle with singularities at codimension two. (For example, a vector bundle in which an instanton has become small is associated with a torsion-free sheaf.)

Given a sheaf $\mathcal{E}$, we can define its dual $\mathcal{E}^{\vee}=\operatorname{Hom}(\mathcal{E}, \mathcal{O})$. A reflexive sheaf $\mathcal{E}$ is a sheaf isomorphic to its double dual $\mathcal{E}^{\vee \vee}$, i.e., $\mathcal{E}=\mathcal{E}^{\vee \vee}$. In general, for any torsion-free sheaf $\mathcal{E}, \mathcal{E}^{\vee \vee}$ and even $\mathcal{E}^{\vee}$ are reflexive, regardless of whether $\mathcal{E}$ was reflexive. On a smooth variety, reflexive sheaves are locally free except on a codimension three subvariety.

Thus, a reflexive sheaf is also torsion-free, and a locally free sheaf is both reflexive and torsion-free.

On a smooth variety, a reflexive rank 1 sheaf is precisely a line bundle.

Over singular varieties this description is sometimes misleading. For example, on a singular curve it is possible to have sheaves which are torsion-free but not locally free. Also, on a singular variety it is possible to have reflexive rank 1 sheaves which are not line bundles (see for example section 4.5.5).

The nomenclature "locally free," "torsion-free," and "reflexive" refers to properties of the stalks of the sheaf. For a sheaf to be locally free means precisely each stalk is a freely generated module, torsion-free means each stalk is torsion-free, and reflexive means each stalk is reflexive. (These definitions hold even on singular varieties.)

Deformations of a vector bundle $\mathcal{E}$ are classified by elements of $H^{1}($ End $\mathcal{E}$ ). When $\mathcal{E}$ is a more general sheaf, its deformations are classified by (global) $\operatorname{Ext}^{1}(\mathcal{E}, \mathcal{E})[41]$. (As the reader may guess, on a Calabi-Yau surface the Yoneda pairing $\operatorname{Ext}^{1}(\mathcal{E}, \mathcal{F}) \times \operatorname{Ext}^{1}(\mathcal{F}, \mathcal{G}) \rightarrow \operatorname{Ext}^{2}(\mathcal{E}, \mathcal{G})$ defines a symplectic structure on the moduli space of sheaves [45].)

For more information on sheaf theory and sheaf-theoretic homological algebra, see for example [38, 107] and also [13, 14].

\section{GIT Quotients}

Geometric Invariant Theory quotients are described in more detail in [46, $47,40]$ and [80, section 4.2]. 
A GIT quotient $\mathcal{T} / / G$ should be thought of as closely related to the quotient space $\mathcal{T} / G$. The difference is that before taking the quotient, some subset (the "unstable points") of $\mathcal{T}$ is removed. Less often, some properly semistable points are identified.

An elementary example should be useful. The space $\mathbf{P}^{n}$ can be described as a GIT quotient of the space $\mathbf{C}^{n+1}$ by $\mathbf{C}^{\times}$. More precisely,

$$
\mathbf{P}^{n}=\frac{\left(\mathbf{C}^{n+1}-0\right)}{\mathbf{C}^{\times}}
$$

To specify the unstable points of $\mathcal{T}$ (the points to be omitted before quotienting) one must first specify an ample line bundle $\mathcal{L}$, called the polarization. Then,

$$
\mathcal{T} / / G=\operatorname{Proj}\left(\bigoplus_{n \geq 0} H^{0}\left(\mathcal{T}, \mathcal{L}^{n}\right)^{G}\right)
$$

Points of $\mathcal{T}$ are classified as unstable, semistable, or stable. The unstable points are precisely the points omitted from $\mathcal{T}$ before quotienting by $G$. These unstable points are points $x$ such that for all $G$-invariant sections $\sigma$ of $\mathcal{L}^{n}$, for all $n>0, \sigma(x)=0$. Semistable points of $\mathcal{T}$ are points $x \in \mathcal{T}$ such that there exists a $G$-invariant section $\sigma \in H^{0}\left(\mathcal{T}, \mathcal{L}^{n}\right)$ for some $n$ such that $\sigma(x) \neq 0$. Stable points of $\mathcal{T}$ are precisely semistable points with finite stabilizer and the property that in the set of semistable points, their $G$-orbit is closed.

Intuitively, why is this definition sensible? Suppose that $\mathcal{L}$ is a very ample line bundle on $\mathcal{T}$, then more or less by definition of very ample,

$$
\mathcal{T} \cong \operatorname{Proj} \bigoplus_{n / g e q 0} H^{0}\left(\mathcal{T}, \mathcal{L}^{n}\right)
$$

If $G$ acts freely on $\mathcal{T}$, then

$$
\mathcal{T} / G \cong \operatorname{Proj}\left(\bigoplus_{n \geq 0} H^{0}\left(\mathcal{T}, \mathcal{L}^{n}\right)^{G}\right)
$$

It should now seem quite reasonable that when the action of $G$ is not so well-behaved, one recovers the GIT quotient outlined above.

Note that the language defined above coincides with the language used to define moduli spaces of sheaves. In both cases, one speaks of stable, semistable, and unstable objects, and in particular before forming the moduli space one removes the unstable objects. This is not a coincidence. Historically, GIT quotients were originally developed in large part to study moduli 
spaces of sheaves on curves. In particular, in the text we show that the moduli spaces of equivariant sheaves on a toric variety are precisely GIT quotients. This partially justifies the terrible notation, in which points with big (positive-dimensional) stabilizers in the group are the least "stable" !

In fact we have been somewhat sloppy. We must also specify a (linearized) action of $G$ on the ample line bundle $\mathcal{L}$. Let $\pi: \mathcal{L} \rightarrow \mathcal{T}$ be the bundle projection, then we say [47, p. 81] a linearized action of $G$ is an action such that $\mathcal{L}$ is $G$-equivariant, meaning

$$
\pi(g y)=g \pi(y) \text { for all } y \in \mathcal{L}, g \in G
$$

and in addition to being $G$-equivariant, for all $x \in \mathcal{T}, g \in G$, the map

$$
\mathcal{L}_{x} \rightarrow \mathcal{L}_{g x}: y \mapsto g y
$$

must be linear. The second condition is equivalent to saying that $G$ acts linearly on the homogeneous coordinates of the projective space in which $\mathcal{T}$ is embedded via $\mathcal{L}$ (or a tensor power of $\mathcal{L}$, if it is not very ample).

In practice there is a more nearly straightforward way to test stability of a point of $\mathcal{T}$, known as the numerical criterion for stability (see [47, section 4.2] or [46, section 2.1]). Let $x \in \mathcal{T}$, and $\hat{x} \in \mathrm{P}^{n}$ the image of $x$ under the projective embedding defined by $\mathcal{L}$. Write $\hat{x}$ in terms of homogeneous coordinates as $\hat{x}=\left(\hat{x}_{0}, \hat{x}_{1}, \cdots, \hat{x}_{n}\right)$. Let $\lambda$ be a one-parameter subgroup of $G$, whose action on $x$ is determined by its action on $\hat{x}$ as

$$
\lambda(t) \hat{x}=\left(t^{r_{0}} \hat{x}_{0}, t^{r_{1}} \hat{x}_{1}, \cdots, t^{r_{n}} \hat{x}_{n}\right)
$$

then define

$$
\begin{aligned}
\mu(x, \lambda) & =\text { unique integer } \mu \text { such that } \lim _{t \rightarrow 0} t^{\mu} \lambda(t) \hat{x} \text { exists and is nonzero } \\
& =\max \left\{-r_{i} \mid \hat{x}_{i} \neq 0\right\}
\end{aligned}
$$

(The reader should not confuse the $\mu$ above with the $\mu$ used to denote the slope of a sheaf. Unfortunately it is standard in the mathematics literature to use $\mu$ to denote both, and we have chosen to abide by their conventions.) Then, it can be shown that $x$ is semistable precisely when $\mu(x, \lambda) \geq 0$ for all one-parameter subgroups $\lambda$ of $G$, and $x$ is stable precisely when $\mu(x, \lambda)>0$ for all one-parameter subgroups $\lambda$ of $G$.

Intuitively, why should the numerical criterion for stability be correct? First, it should be intuitively clear ${ }^{44}$ that $x$ is semistable precisely when the

\footnotetext{
${ }^{44}$ In fact, it is rigorously correct.
} 
closure of the $G$-orbit of $\hat{x}$ does not intersect 0 . Then, note that if 0 is not in the closure of the $G$-orbit of $\hat{x}$, then for all one-parameter subgroups $\lambda(t)$ of $G$, it must be the case that

$$
\lim _{t \rightarrow 0} \lambda(t) \hat{x} \neq 0
$$

and from this the numerical criterion for stability follows immediately.

A useful fact is that if we have specified several identical spaces $\mathcal{T}$, with a projective embedding defined by composing the projective embeddings defined by the $\mathcal{L}$ 's over each $\mathcal{T}$ with a Segre embedding, then

$$
\mu\left(\left(x_{1}, \cdots, x_{N}\right), \lambda\right)=\sum_{i=1}^{N} \mu\left(x_{i}, \lambda\right)
$$

for all one-parameter subgroups $\lambda$ of $G$ ([47, lemma 4.12]). This is relatively straightforward to demonstrate, as we shall now outline. Let $\hat{x}_{1}, \hat{x}_{2}$ denote sets of homogeneous coordinate for either of two projective embeddings, and suppose we have a one-parameter subgroup action $\lambda(t)$ defined by

$$
\begin{aligned}
& \lambda(t) \hat{x}_{1}=\left(t^{r_{10}} \hat{x}_{10}, t^{r_{11}} \hat{x}_{11}, \cdots, t^{r_{1 n}} \hat{x}_{1 n}\right) \\
& \lambda(t) \hat{x}_{2}=\left(t^{r_{20}} \hat{x}_{20}, t^{r_{21}} \hat{x}_{21}, \cdots, t^{r_{2 m}} \hat{x}_{2 m}\right)
\end{aligned}
$$

The action of $\lambda$ on the homogeneous coordinates of the Segre embedding is clear:

$$
\lambda(t)\left(\hat{x}_{1 i} \hat{x}_{2 j}\right)=t^{r_{1 i}+r_{2 j}} \hat{x}_{1 i} \hat{x}_{2 j}
$$

so we can now compute

$$
\begin{aligned}
\mu(x, \lambda) & =\max \left\{-r_{1 i}-r_{2 j} \mid \hat{x}_{1 i} \hat{x}_{2 j} \neq 0\right\} \\
& =\mu\left(x_{1}, \lambda\right)+\mu\left(x_{2}, \lambda\right)
\end{aligned}
$$

just as stated.

The resulting GIT quotient depends not only upon the choice of ample line bundle $\mathcal{L}$, but also upon the choice of $G$-linearization [55, 56]-different choices of either the ample line bundle or $G$-linearization may yield distinct results.

For example, let us consider a simple example of a flop. Let $a_{1}, a_{2}, b_{1}, b_{2}$ be coordinates on $\mathbf{C}^{4}$. Define a $\mathbf{C}^{\times}$action $\lambda$ on $\mathbf{C}^{4}$ by

$$
\begin{aligned}
& a_{1,2} \rightarrow \lambda a_{1,2} \\
& b_{1,2} \rightarrow \lambda^{-1} b_{1,2}
\end{aligned}
$$


and define

$$
\begin{aligned}
& x=a_{1} b_{1} \\
& y=a_{2} b_{2} \\
& z=a_{1} b_{2} \\
& t=a_{2} b_{1}
\end{aligned}
$$

We fix the action of $G=\mathbf{C}^{\times}$on $\mathbf{C}^{4}$ and vary the $G$-linearization of $\mathcal{O}$; we shall see shortly that this reproduces the expected distinct quotients.

For convenience, we shall define some notation. Let $R=H^{0}\left(\mathbf{C}^{4}, \mathcal{O}\right)$, and give it a grading determined by weight under the action of $G=\mathbf{C}^{\times}$. Then,

$$
\begin{aligned}
R_{0} & =H^{0}\left(\mathbf{C}^{4}, \mathcal{O}\right)^{G} \\
& =\mathbf{C}[x, y, z, t] /(x y-z t)
\end{aligned}
$$

and also define the graded rings

$$
\begin{aligned}
R_{\geq 0} & =\bigoplus_{n \geq 0} R_{n} \\
& =\mathbf{C}[x, y, z, t]\left[a_{1}, a_{2}\right] /\left(a_{1} y-a_{2} z, a_{1} t-a_{2} x\right) \\
R_{\leq 0} & =\bigoplus_{n \geq 0} R_{-n} \\
& =\mathbf{C}[x, y, z, t]\left[b_{1}, b_{2}\right] /\left(b_{1} z-b_{2} x, b_{1} y-b_{2} t\right)
\end{aligned}
$$

where $a_{1}, a_{2}$ should be considered as homogeneous variables defining the grading of $R_{\geq 0}$, and $b_{1}, b_{2}$ defining the grading of $R_{\leq 0}$.

Now, although as line bundles $\mathcal{O}^{n}$ and $\mathcal{O}$ are identical for all $n \geq 0$, the $G$-linearizations of each differ (unless, of course, the $G$-linearization of $\mathcal{O}$ is trivial).

Formally, write

$$
\bigoplus_{n \geq 0} H^{0}\left(\mathbf{C}^{4}, \mathcal{O}^{n}\right)=R[z]
$$

where $z$ is a dummy variable defining the grading. Assume $z$ has weight $-k \in \mathbf{Z}$ under $G$, defining the $G$-linearization of $\mathcal{O}$. Then we have

$$
\begin{aligned}
\operatorname{Proj}\left[\bigoplus_{n \geq 0} H^{0}\left(\mathbf{C}^{4}, \mathcal{O}^{n}\right)\right]^{G} & =\operatorname{Proj} R[z]^{G} \\
& =\operatorname{Proj}\left[\bigoplus_{n \geq 0} R_{k n} z^{n}\right]
\end{aligned}
$$




$$
\begin{aligned}
& = \begin{cases}\text { Proj } \bigoplus_{n \geq 0} R_{n} z^{n} & k>0 \\
\text { Proj } R_{0}[z] & k=0 \\
\text { Proj } \oplus_{n \geq 0} R_{-n} z^{n} & k<0\end{cases} \\
& = \begin{cases}\text { Proj } R_{\geq 0} & k>0 \\
\text { Spec } R_{0} & k=0 \\
\text { Proj } R_{\leq 0} & k<0\end{cases}
\end{aligned}
$$

Clearly, the three possible GIT quotients above are precisely the conifold and its two small resolutions. For $k>0$, the unstable set on $\mathbf{C}^{4}$ is precisely $\left\{a_{1}=a_{2}=0\right\}$, and for $k<0$ the unstable set is precisely $\left\{b_{1}=b_{2}=0\right\}$.

Finally, we should mention that GIT quotients are equivalent to symplectic quotients (see [48] or the appendix to [46] for more information). Suppose we are constructing the GIT quotient of a space $\mathcal{T}$ by the action of a reductive algebraic group $G$ with maximal compact subgroup $K$. Let $\iota: \mathcal{T} \rightarrow \mathbf{P}^{n}$ denote the projective embedding defined by polarization $\mathcal{L}$. Then to define the equivalent symplectic quotient, we take the symplectic form ${ }^{45}$ to be the restriction of the Kähler form from the projective embedding, and the moment map is defined by the composition

$$
\mathcal{T} \stackrel{\iota}{\longrightarrow} \mathbf{P}^{n} \stackrel{\Phi_{1}}{\longrightarrow} p u(n+1)^{*} \stackrel{\Phi_{2}}{\longrightarrow} k^{*}
$$

where $\Phi_{1}$ is the moment map for the action of $P U(n+1)$ on $\mathbf{P}^{n}, \Phi_{2}$ is the transpose of the differential of the map of $K \subset G$ into $P U(n+1)$, and $k=$ Lie $K$. The corresponding symplectic quotient is then

$$
\frac{\left(\Phi_{2} \circ \Phi_{1} \circ \iota\right)^{-1}(0)}{K}
$$

Note that not all symplectic quotients have equivalent GIT quotients. For example, one necessary (but not sufficient) condition is that the symplectic form be (the image of) an element of $H^{2}(\mathbf{Z})$.

\section{Filtrations}

One important filtration of a coherent sheaf, often appearing in the mathematics literature, is called the Harder-Narasimhan filtration. Given a torsionfree coherent sheaf $\mathcal{E}$, a Harder-Narasimhan filtration of $\mathcal{E}$ is a filtration [74]

$$
0=\mathcal{E}_{0} \subset \mathcal{E}_{1} \subset \cdots \subset \mathcal{E}_{n}=\mathcal{E}
$$

\footnotetext{
${ }^{45}$ This symplectic form also happens to be a de Rham representative of the cohomology class $c_{1}(\mathcal{L})$. However, not all representatives of $c_{1}(\mathcal{L})$ yield possible symplectic forms.
} 
(where $\subset$ indicates proper subset) with the properties

(1) $\mathcal{E}_{i} / \mathcal{E}_{i-1}$ is semistable for $1 \leq i \leq n$

(2) $\mu\left(\mathcal{E}_{i} / \mathcal{E}_{i-1}\right)>\mu\left(\mathcal{E}_{i+1} / \mathcal{E}_{i}\right)$ for $1 \leq i \leq n-1$

For every torsion-free coherent sheaf, a Harder-Narasimhan filtration exists and is unique.

Note that the Harder-Narasimhan filtration is trivial (meaning, of the form $0=\mathcal{E}_{0} \subset \mathcal{E}_{1}=\mathcal{E}$ ) precisely when $\mathcal{E}$ is semistable. Intuitively, the Harder-Narasimhan filtration gives information about the "instability" of $\mathcal{E}$.

It is quite easy to derive the Harder-Narasimhan filtration for any torsionfree coherent sheaf $\mathcal{E}$. Let $\mathcal{E}_{1}$ be a destabilizing subsheaf (meaning, $\mu\left(\mathcal{E}_{1}\right)>$ $\mu(\mathcal{E})$ ) of greatest possible slope, such that among all destabilizing subsheaves of that slope, $\mathcal{E}_{1}$ has the greatest rank. Now, let $\mathcal{E}_{2}$ be a subsheaf of $\mathcal{E}$ such that $\mathcal{E}_{1} \subset \mathcal{E}_{2}$, and so that $\mathcal{E}_{2} / \mathcal{E}_{1}$ has maximal slope among subsheaves of $\mathcal{E} / \mathcal{E}_{1}$, and such that among such subsheaves of equal slope, it has maximal rank. Proceeding in this manner, one can construct each element of the Harder-Narasimhan filtration.

Another important kind of filtration worth mentioning is a Jordan-Hölder filtration. Just as the Narasimhan-Harder filtration splits any torsion-free coherent sheaf into semistable factors, a Jordan-Hölder filtration splits a semistable sheaf into stable factors.

More precisely, let $\mathcal{E}$ be a semistable sheaf. A Jordan-Hölder filtration of $\mathcal{E}$ is a filtration

$$
0=\mathcal{E}_{0} \subset \mathcal{E}_{1} \subset \cdots \subset \mathcal{E}_{n}=\mathcal{E}
$$

such that the factors $\mathcal{E}_{i} / \mathcal{E}_{i+1}$ are stable.

Jordan-Hölder filtrations always exist, but are not necessarily unique.

Although the Jordan-Hölder filtration is not necessarily unique, the associated graded filtration defined by $g r_{i}(\mathcal{E})=\mathcal{E}_{i} / \mathcal{E}_{i+1}$ is unique. Two semistable sheaves with isomorphic associated graded filtrations are S-equivalent.

What is S-equivalence? Recall from the discussion of GIT quotients that sometimes properly semistable points are identified in forming the quotient, so only equivalence classes of properly semistable objects appear in the GIT quotient. In a moduli space of sheaves, such an equivalence class of properly semistable sheaves is said to be an S-equivalence class, and two properly semistable sheaves belonging to the same S-equivalence class (meaning, appearing at the same point on the moduli space) are said to be S-equivalent. 
In the language of symplectic quotients, $S$-equivalence classes occur when one performs symplectic reduction at a nonregular value of the moment map.

Thus, Jordan-Hölder filtrations are useful for determining S-equivalence classes of properly semistable sheaves.

\section{References}

[1] A. A. Klyachko, "Toric Bundles and Problems of Linear Algebra," Funct. Anal. Appl. 23 (1989) 135.

[2] A. A. Klyachko, "Equivariant Bundles on Toral Varieties," Math. USSR Izvestiya 35 (1990) 337.

[3] A. A. Klyachko, "Stable Bundles, Representation Theory and Hermitian Operators," preprint.

[4] A. A. Klyachko, "Vector Bundles and Torsion Free Sheaves on the Projective Plane," preprint.

[5] A. A. Klyachko, "Spatial Polygons and Stable Configurations of Points in the Projective Line," in Algebraic Geometry and its Applications: Proceedings of the 8th Algebraic Geometry Conference, Yaroslavl' 1992, ed. A. Tikhomirov and A. Tyurin, Vieweg, Bonn, 1992.

[6] T. Kaneyama, "On Equivariant Vector Bundles on an Almost Homogeneous Variety," Nagoya Math. J. 57 (1975) 65.

[7] T. Kaneyama, "Torus-Equivariant Vector Bundles on Projective Spaces," Nagoya Math. J. 111 (1988) 25.

[8] W. Fulton, Introduction to Toric Varieties, Princeton University Press, 1993.

[9] T. Oda, Convex Bodies and Algebraic Geometry, Springer-Verlag, 1985.

[10] G. Kempf, F. Knudsen, D. Mumford, and B. Saint-Donat, Toroidal Embeddings I, Lecture Notes in Mathematics 339, Springer-Verlag, 1973.

[11] V. I. Danilov, "The Geometry of Toric Varieties," Russian Math Surveys 33:2 (1978) 97. 
[12] D. A. Cox, "The Homogeneous Coordinate Ring of a Toric Variety," J. Algebraic Geom. 4 (1995) 17, alg-geom/9210008.

[13] E. Sharpe, "Extremal Transitions in Heterotic String Theory," PUPT1707, hep-th/9705210.

[14] E. Sharpe, "Notes on Heterotic Compactifications," PUPT-1729, hep-th/9710031.

[15] E. Sharpe, "Extremal Transitions and $(0,2)$ Models," in preparation.

[16] A. Knutson, E. Sharpe, in preparation.

[17] J. Distler and S. Kachru, "(0,2) Landau-Ginzburg Theory," Nucl. Phys. B413 (1994) 213, hep-th/9309110; J. Distler, "Notes on $(0,2)$ Superconformal Field Theories," hep-th/9502012.

[18] X.-G. Wen and E. Witten, "World-Sheet Instantons and the PecceiQuinn Symmetry," Phys. Lett. 166B (1986) 397; M. Dine, N. Seiberg, X.-G. Wen, and E. Witten, "Nonperturbative Effects on the String World Sheet I, II," Nucl. Phys. B278 (1986) 769; Nucl. Phys. B289 (1987) 319.

[19] E. Silverstein and E. Witten, "Criteria for Conformal Invariance of $(0,2)$ Models," Nucl. Phys. B444 (1995) 161, hep-th/9503212.

[20] J. Distler and B. Greene, "Aspects of $(2,0)$ String Compactifications," Nucl. Phys. B304 (1988) 1.

[21] E. Witten, "Phases of $\mathrm{N}=2$ Theories in Two Dimensions," Nucl. Phys. B403 (1993) 159, hep-th/9301042.

[22] J. Distler and S. Kachru, "Quantum Symmetries and Stringy Instantons," Phys. Lett. B336 (1994) 368, hep-th/9406091.

[23] J. Distler, B. Greene, and D. Morrison, "Resolving Singularities in $(0,2)$ Models," Nucl. Phys. B481 (1996) 289, hep-th/9605222.

[24] M. Duff, R. Minasian, and E. Witten, "Evidence for Heterotic / Heterotic Duality," Nucl. Phys. B465 (1996) 413, hep-th/9601036.

[25] P. Aspinwall, B. Greene, and D. Morrison, "Calabi-Yau Moduli Space, Mirror Manifolds, and Spacetime Topology Change in String Theory," Nucl. Phys. B416 (1994) 414, hep-th/9309097.

[26] S. Kobayashi, Differential Geometry of Complex Vector Bundles, Princeton University Press, Princeton, 1987. 
[27] S. K. Donaldson, "Anti Self Dual Yang-Mills Connections over Complex Algebraic Surfaces and Stable Vector Bundles," Proc. London Math. Soc. (3) 50 (1985) 1.

[28] K. Uhlenbeck and S. T. Yau, "On the Existence of Hermitian YangMills Connections in Stable Vector Bundles," Comm. Pure Appl. Math. 39 (1986) S257.

[29] R. Friedman, J. Morgan, and E. Witten, "Vector Bundles and F Theory," hep-th/9701162

[30] R. Friedman, J. Morgan, and E. Witten, "Principal G-Bundles over Elliptic Curves," alg-geom/9707004.

[31] R. Friedman, J. Morgan, and E. Witten, "Vector Bundles over Elliptic Fibrations," alg-geom/9709029.

[32] M. Bershadsky, A. Johansen, T. Pantev, and V. Sadov, "On FourDimensional Compactifications of F-Theory," hep-th/9701165

[33] R. Donagi, "Principal Bundles on Elliptic Fibrations," alg-geom/9702002

[34] B. Andreas, G. Curio, and D. Lüst, "N=1 Dual String Pairs and their Massless Spectra," hep-th/9705174.

[35] P. Aspinwall and D. Morrison, "Point-like Instantons on $K 3$ Orbifolds," hep-th/9705104.

[36] E. Looijenga, "Root Systems and Elliptic Curves," Invent. Math. 38 (1977) 17.

[37] E. Looijenga, "Invariant Theory for Generalized Root Systems," Invent. Math. 61 (1980) 1.

[38] C. Okonek, M. Schneider, and H. Spindler, Vector Bundles on Complex Projective Spaces, Birkhauser, 1980.

[39] F. Takemoto, "Stable Vector Bundles on Algebraic Surfaces," Nagoya Math. J. 47 (1972) 29.

[40] M. Maruyama, "Moduli of Stable Sheaves, I," J. Math. Kyoto 17 (1977) 91.

[41] M. Maruyama, "Moduli of Stable Sheaves, II," J. Math. Kyoto 18 (1978) 557. 
[42] G. Horrocks, "Construction of Bundles on $\mathbf{P}^{n}$," in Les Équations de Yang-Mills, ed. by A. Douady, J.-L. Verdier, Séminaire E.N.S 19771978, 1980.

[43] W. Barth and K. Hulek, "Monads and Moduli of Vector Bundles," Manuscripta Math. 25 (1978) 323.

[44] A. A. Beilinson, "Coherent Sheaves on $\mathrm{P}^{n}$ and Problems of Linear Algebra," Funct. Anal. Appl. 12 (1978) 214.

[45] S. Mukai, "Symplectic Structure of the Moduli Space of Sheaves on an Abelian or K3 Surface," Invent. Math. 77 (1984) 101.

[46] D. Mumford, J. Fogarty, and F. Kirwan, Geometric Invariant Theory, third edition, Springer-Verlag, 1994.

[47] P. E. Newstead, Lectures on Introduction to Moduli Problems and Orbit Spaces, Tata Institute of Fundamental Research, Springer-Verlag, Berlin, 1978.

[48] F. Kirwan, Cohomology of Quotients in Symplectic and Algebraic Geometry, Princeton University Press, 1984.

[49] A. Białynicki-Birula, "Some Theorems on Actions of Algebraic Groups," Ann. Math. 98 (1973) 480.

[50] V. A. Ginzburg, "Equivariant Cohomologies and Kähler's Geometry," Funct. Anal. Appl. 21 (1987) 271.

[51] J. Humphreys, Linear Algebraic Groups, Springer-Verlag, New York, 1981.

[52] A. Borel, Linear Algebraic Groups, W. A. Benjamin, New York, 1969.

[53] J. C. Jantzen, Representations of Algebraic Groups, Academic Press, Boston, 1987.

[54] Y. Hu, "(W-R) Matroids and Thin Schubert-Type Cells Attached to Algebraic Torus Actions," Proc. Amer. Math. Soc. 123 (1995) 2607.

[55] I. Dolgachev and Y. Hu, "Variation of Geometric Invariant Theory Quotients," alg-geom/9402008.

[56] M. Thaddeus, "Geometric Invariant Theory and Flips," J. Amer. Math. Soc. 9 (1996) 691.

[57] M. Reid, "Canonical 3-Folds," in Journées de Géometrié Algébrique d'Angers, ed. by A. Beauville, Sijthoff and Noordhoff, Alphen aan den Rijn, 1980, pp. 273-310. 
[58] D. A. Cox, "Recent Developments in Toric Geometry," alg-geom/9606016.

[59] M. Atiyah, "Convexity and Commuting Hamiltonians," Bull. London Math. Soc. 14 (1982) 1.

[60] V. Guillemin and S. Sternberg, "Convexity Properties of the Moment Mapping," Invent. Math. 67 (1982) 491.

[61] M. F. Atiyah and R. S. Ward, "Instantons and Algebraic Geometry," Comm. Math. Phys. 55 (1977) 117.

[62] V. G. Drinfel'd and Yu. I. Manin, "Self-Dual Yang-Mills Fields over a Sphere," Funct. Anal. Appl. 12 (1978) 140.

[63] Z. Qin, "Chamber Structures of Algebraic Surfaces with Kodaira Dimension Zero and Moduli Spaces of Stable Rank Two Bundles," Math. Z. 207 (1991) 121.

[64] Z. Qin, "Birational Properties of Moduli Spaces of Stable Locally Free Rank-2 Sheaves on Algebraic Surfaces," Manuscripta Math. 72 (1991) 163.

[65] Z. Qin, "Equivalence Classes of Polarizations and Moduli Spaces of Sheaves," J. Diff. Geom. 37 (1993) 397.

[66] Z. Qin, "Moduli of Stable Sheaves on Ruled Surfaces and their Picard Groups," J. reine angew. Math. 433 (1992) 201.

[67] R. Friedman and Z. Qin, "On Complex Surfaces Diffeomorphic to Rational Surfaces," Invent. Math. 120 (1995) 81.

[68] K. Matsuki and R. Wentworth, "Mumford-Thaddeus Principle on the Moduli Space of Vector Bundles on an Algebraic Surface," Int. J. Math. 8 (1997) 97.

[69] Y. Hu and W. Li, "Variation of the Gieseker and Uhlenbeck Compactifications," Int. J. Math. 6 (1995) 397.

[70] L. Göttsche, "Change of Polarization and Hodge Numbers of Moduli Spaces of Torsion Free Sheaves on Surfaces," Math. Z. 223 (1996) 247.

[71] R. Friedman and Z. Qin, "Flips of Moduli Spaces and Transition Formulas for Donaldson Polynomial Invariants of Rational Surfaces," Comm. Anal. Geom. 3 (1995) 11.

[72] G. Moore and E. Witten, "Integration over the $u$-Plane in Donaldson Theory," hep-th/9709193. 
[73] W. Barth, "Some Properties of Stable Rank-2 Vector Bundles on $\mathbf{P}^{n}$," Math. Ann. 226 (1977) 125.

[74] M. Maruyama, "Boundedness of Semi-Stable Sheaves of Small Ranks," Nagoya Math. J. 78 (1980) 65.

[75] S. K. Donaldson, "Instantons and Geometric Invariant Theory," Comm. Math. Phys. 93 (1984) 453.

[76] K. Hulek, "On the Classification of Stable Rank $r$ Vector Bundles over the Projective Plane," in Vector Bundles and Differential Equations, ed. A. Hirschowitz, Birkhauser, Boston, 1980.

[77] W. Barth, "Moduli of Vector Bundles on the Projective Plane," Inv. Math. 42 (1977) 63.

[78] M. Maruyama, "Vector Bundles on $\mathbf{P}^{2}$ and Torsion Sheaves on the Dual Plane," in Vector Bundles on Algebraic Varieties, Tata Institute of Fundamental Research, Oxford University Press, Bombay, 1987.

[79] K. Hulek, "Stable Rank-2 Vector Bundles on $\mathbf{P}^{2}$ with $c_{1}$ Odd," Math. Ann. 242 (1979) 241.

[80] D. Huybrechts and M. Lehn, The Geometry of Moduli Spaces of Sheaves, Vieweg, Bonn, 1997.

[81] H. Bass and W. Haboush, "Linearizing of Certain Reductive Group Actions," Trans. Amer. Math. Soc. 292 (1985) 463.

[82] H. Kraft, "Algebraic Automorphisms of Affine Space," Prog. in Math. 80 (1989) 81.

[83] M. Brion, "Equivariant Chow Groups for Torus Actions," preprint.

[84] M. F. Atiyah and I. G. MacDonald, Introduction to Commutative Algebra, Addison-Wesley, Reading, Massachusetts, 1969.

[85] M. F. Atiyah, "Vector Bundles over an Elliptic Curve," Proc. London Math. Soc. (3) 7 (1957) 414.

[86] V. Mehta, "On Some Restriction Theorems for Semistable Bundles," in Invariant Theory, Lecture Notes in Mathematics 996, Springer-Verlag, Berlin, 1983.

[87] H. Flenner, "Restrictions of Semistable Bundles on Projective Varieties," Comment. Math. Helvetici 59 (1984) 635. 
[88] V. Mehta and A. Ramanathan, "Semistable Sheaves on Projective Varieties and their Restrictions to Curves," Math. Ann. 258 (1982) 213.

[89] V. Mehta and A. Ramanathan, "Restriction of Stable Sheaves and Representations of the Fundamental Group," Invent. Math. 77 (1984) 163.

[90] V. V. Batyrev, "Dual Polyhedra and Mirror Symmetry for Calabi-Yau Hypersurfaces in Toric Varieties," J. Alg. Geom. 3 (1994) 493.

[91] R. Blumenhagen, "(0,2) Target Space Duality, CICYs and Reflexive Sheaves," hep-th/9710021.

[92] R. Blumenhagen and S. Sethi, "On Orbifolds of $(0,2)$ Models," hep-th/9611172.

[93] R. Blumenhagen and M. Flohr, "Aspects of $(0,2)$ Orbifolds and Mirror Symmetry," hep-th/9702199.

[94] R. Blumenhagen, R. Schimmrigk, and A. Wisskirchen, "(0,2) Mirror Symmetry," hep-th/9609167.

[95] A. Strominger, S.-T. Yau, and E. Zaslow, "Mirror Symmetry is TDuality," Nucl. Phys. B479 (1996) 243, hep-th/9606040.

[96] D. Morrison, "The Geometry Underlying Mirror Symmetry," alg-geom/9608006.

[97] M. Gross and P. M. H. Wilson, "Mirror Symmetry via 3-Tori for a Class of Calabi-Yau Threefolds," alg-geom/9608004.

[98] M. Gross, "Special Lagrangian Fibrations I: Topology," alg-geom/9710006.

[99] J. M. Figueroa-O'Farrill, A. Imaanpur, and J. McCarthy, "Supersymmetry and Gauge Theory in Calabi-Yau 3-Folds," hep-th/9709178.

[100] S. K. Donaldson and R. P. Thomas, "Gauge Theory in Higher Dimensions," preprint.

[101] R. Thomas, "Gauge Theory on Calabi-Yau Manifolds," Ph.D. thesis, Oxford University, unpublished.

[102] N. C. Leung and C. Vafa, "Branes and Toric Geometry," hep-th/9711013.

[103] R. Bott, "Homogeneous vector bundles," Ann. of Math. 66 (1957) 203. 
[104] E. Witten, "Some Comments on String Dynamics," Strings '95 proceedings, hep-th/9507121.

[105] C. G. Callan, Jr., J. A. Harvey, and A. Strominger, "Worldsheet Approach to Heterotic Instantons and Solitons," Nucl. Phys. B359 (1991) 611.

[106] A. Knutson, in preparation.

[107] R. Hartshorne, Algebraic Geometry, Springer-Verlag, New York, 1977.

[108] R. Friedman, private communication. 